\title{
Modular Design of G-Quadruplex MetalloDNAzymes for Catalytic C-C Bond Formations with Switchable Enantioselectivity
}

Philip M. Punt, ${ }^{\dagger}$ Marie D. Langenberg, ${ }^{\dagger}$ Okan Altan, ${ }^{\dagger}$ and Guido H. Clever*, ${ }^{\dagger}$

'Faculty of Chemistry and Chemical Biology, TU Dortmund University, Otto-Hahn-Str. 6, 44227 Dortmund, Germany.

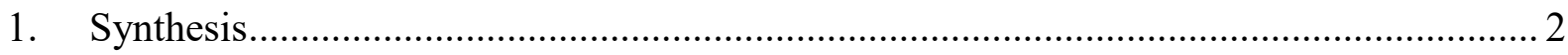

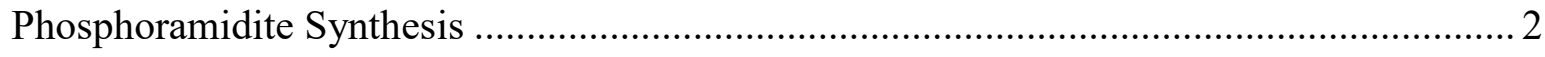

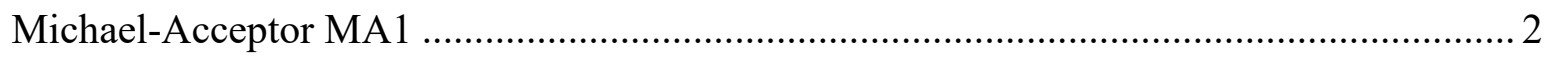

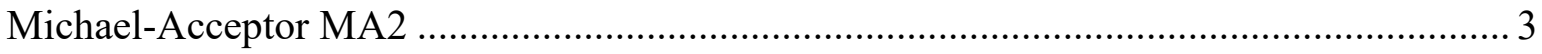

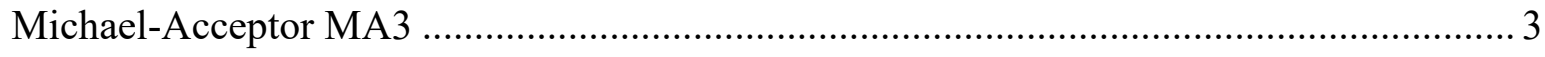

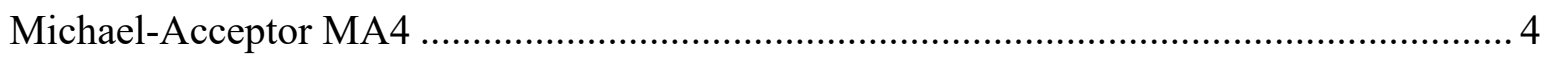

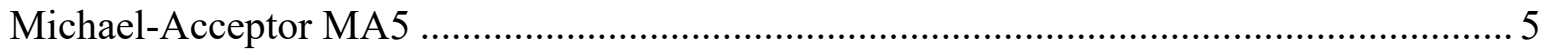

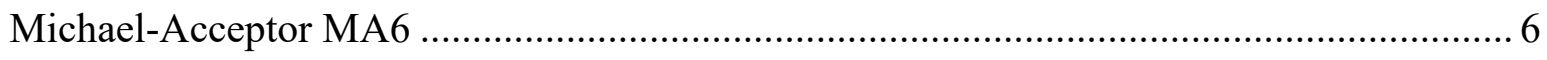

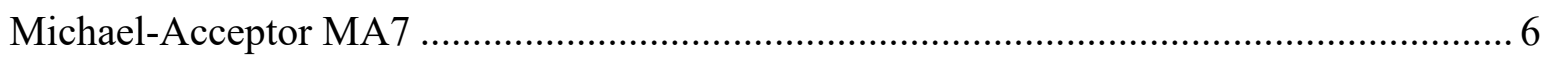

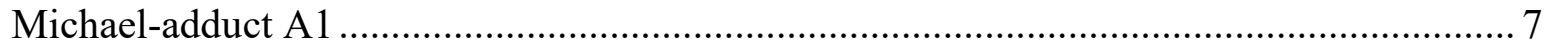

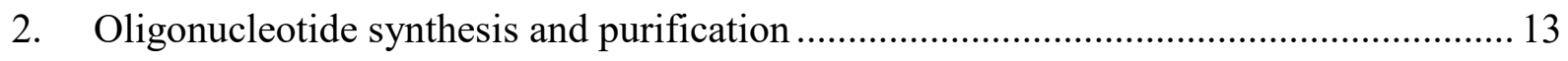

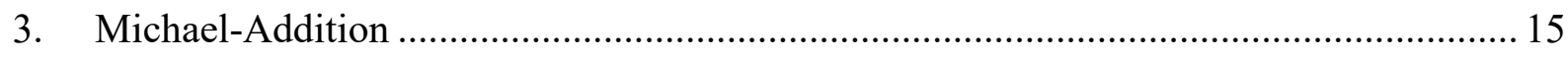

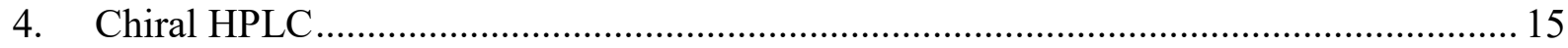

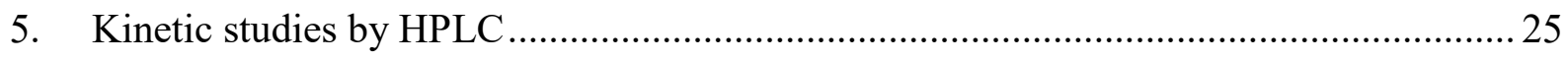

6. Kinetic studies to ascertain Michaelis-Menten saturation behaviour............................. 26

7. UV/VIS and CD spectroscopy and thermal difference spectra ................................. 28

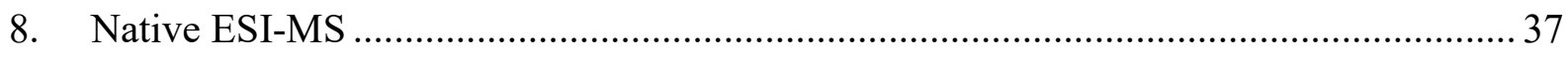

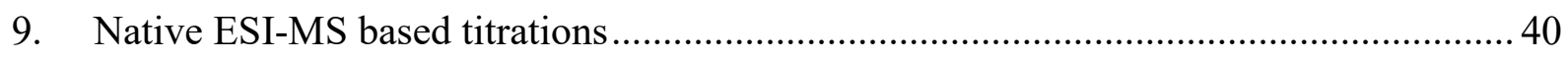

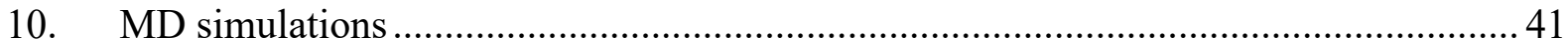

Determination of force field parameters .............................................................. 41

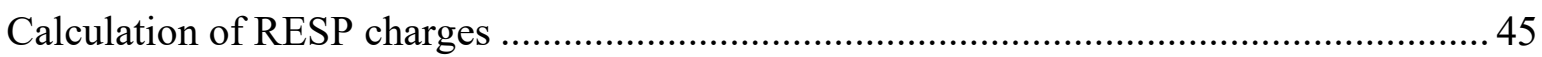

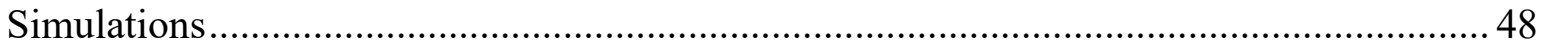

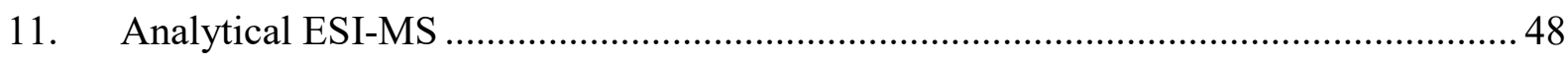

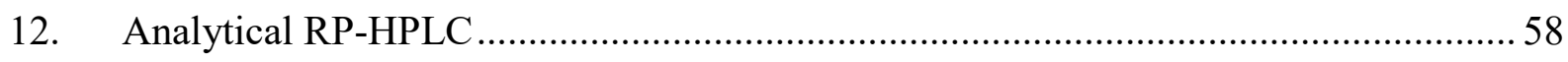




\section{Synthesis}

All chemicals were obtained from commercial sources and used without further purification. Gel permeation chromatography (GPC) purification of ligands was performed on a LC-9210 II NEXT system with $\mathrm{CHCl}_{3}$ (HPLC grade) as eluent. NMR measurements were conducted at $298 \mathrm{~K}$ on Avance-600 and Avance-700 instruments from Bruker and on $500 \mathrm{MHz}$ Bruker Avance neo NMR. Chemical shifts for ${ }^{1} \mathrm{H}$ and ${ }^{13} \mathrm{C}$ are reported in ppm on the $\delta$ scale; ${ }^{1} \mathrm{H}$ and ${ }^{13} \mathrm{C}$ signals were referenced to the residual solvent peak. The following abbreviations are used to describe signal multiplicity for ${ }^{1} \mathrm{H}-\mathrm{NMR}$ spectra: s: singlet, d: doublet, t: triplet, dd: doublet of doublets; dt: doublet of triplets; m: multiplet, br: broad. High resolution electrospray ionization mass spectrometry (ESI HRMS) was performed on a Bruker timsTOF ESI mass spectrometer.

\section{Phosphoramidite Synthesis}

Phosphoramidites needed for DNA solid-phase synthesis were synthesized as previously reported. ${ }^{[1]}$

\section{Michael-Acceptor MA1}
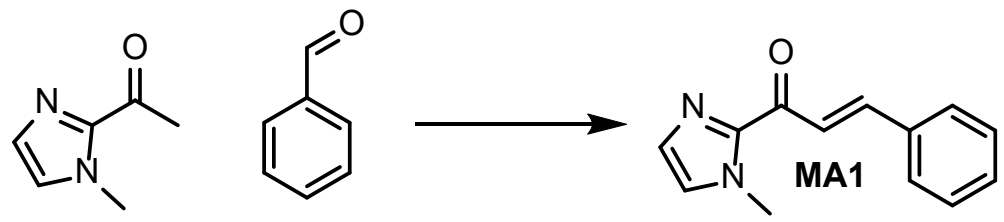

The synthesis of MA1 was adapted from a literature procedure. ${ }^{[2]}$ 1-(1-methyl-1H-imidazol-2-yl)ethan-1-one (340 mg, $2.74 \mathrm{mmol}, 1.1$ equiv.) and benzaldehyde (264 mg, $2.49 \mathrm{mmol}, 1$ equiv.) were added to distilled water (25 $\mathrm{mL})$ and cooled to $4{ }^{\circ} \mathrm{C}$. The mixture was shaken thoroughly to afford a fine emulsion. Then $10 \mathrm{~mL}$ of a $10 \%$ aqueous $\mathrm{NaOH}$ solution was added and the mixture was shaken for $\sim 30$ seconds. The reaction was then left at $4{ }^{\circ} \mathrm{C}$ for $8 \mathrm{~h}$. The resulting white solid was filtered, dried and purified by column chromatography ( $n$ Pentane:EtOAc, 90:10 $\rightarrow 70: 30$ ). The ${ }^{1} \mathrm{H}-$ NMR was in accordance with literature. ${ }^{[3]}$

Chemical Formula: $\mathrm{C}_{13} \mathrm{H}_{12} \mathrm{~N}_{2} \mathrm{O}$

Molecular weight: $212.25 \mathrm{~g} \mathrm{~mol}^{-1}$

HRMS (ESI, pos., Calc. [M+H]+: 213.1022, ACN): Found: m/z=213.1030 [M+H]+, [2M+H]+.

${ }^{1} \mathbf{H}$ NMR $\left(500 \mathrm{MHz}, 298 \mathrm{~K}, \mathrm{CDCl}_{3}\right): \delta(\mathrm{ppm}): 8.15\left(\mathrm{~d},{ }^{3} J=15.9 .0,1 \mathrm{H}\right), 7.84\left(\mathrm{~d},{ }^{3} J=15.9 \mathrm{~Hz}, 1 \mathrm{H}\right), 7.75-7.71(\mathrm{~m}$, 2H), $7.42-7.39(\mathrm{~m}, 3 \mathrm{H}), 7.26\left(\mathrm{~d},{ }^{3} \mathrm{~J}=2.4 \mathrm{~Hz}, 1 \mathrm{H}\right), 7.10(\mathrm{~s}, 1 \mathrm{H}), 4.12(\mathrm{~s}, 3 \mathrm{H})$.

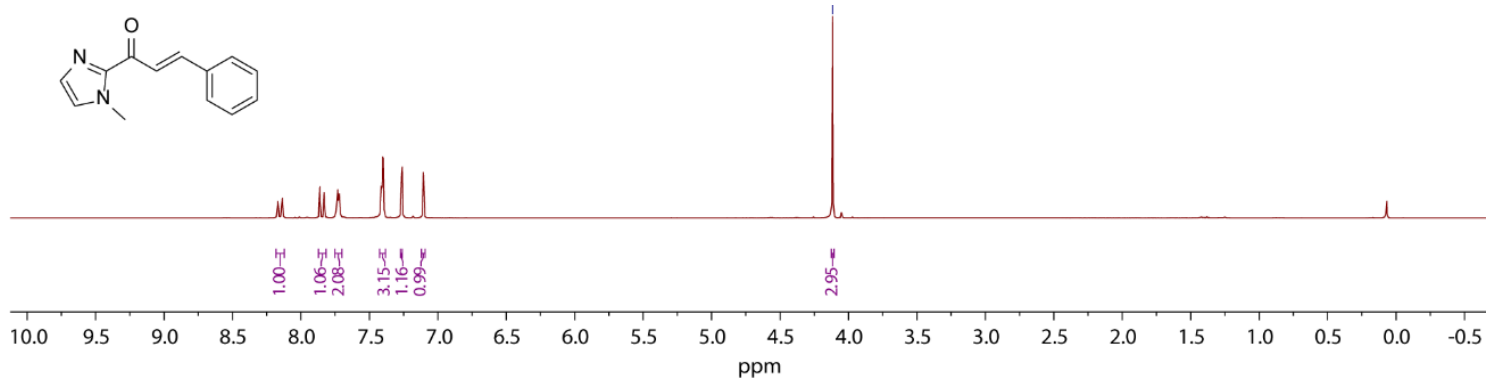

Figure S1. ${ }^{1} \mathrm{H}$ NMR $\left(500 \mathrm{MHz}, 298 \mathrm{~K}, \mathrm{CDCl}_{3}\right)$ of Michael-acceptor MA1. 


\section{Michael-Acceptor MA2}

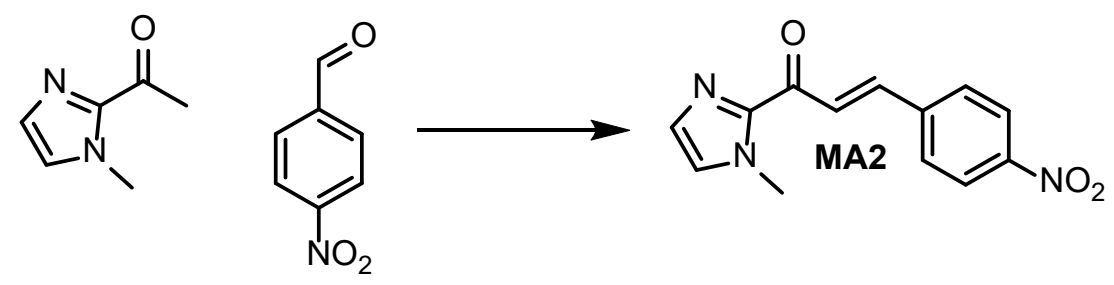

The synthesis of Michael-acceptor MA2 was the same procedure as for Michael-acceptor MA1 (47 mg, $0.18 \mathrm{mmol}$, $50 \%)$. The ${ }^{1} \mathrm{H}$ NMR was in accordance with literature. ${ }^{[4]}$

Chemical Formula: $\mathrm{C}_{13} \mathrm{H}_{11} \mathrm{~N}_{3} \mathrm{O}_{3}$

Molecular weight: $257.25 \mathrm{~g} \mathrm{~mol}^{-1}$

HRMS (ESI, pos., Calc. [M+H]+: 258.0873 ACN): Found: m/z=258.0873 [M+H]+.

${ }^{1} \mathbf{H}$ NMR $\left(500 \mathrm{MHz}, 298 \mathrm{~K}, \mathrm{CDCl}_{3}\right): \delta(\mathrm{ppm}): 8.27-8.24(\mathrm{~m}, 2 \mathrm{H}), 8.20(\mathrm{~d}, J=16.1 \mathrm{~Hz}, 1 \mathrm{H}), 7.85-7.82(\mathrm{~m}, 2 \mathrm{H}) 7.81$ (d, $J=16.1 \mathrm{~Hz}, 1 \mathrm{H}), 7.25$ (d, $J=0.9 \mathrm{~Hz}, 1 \mathrm{H}), 7.13(\mathrm{~s}, 1 \mathrm{H}), 4.11$ (s, 3H).

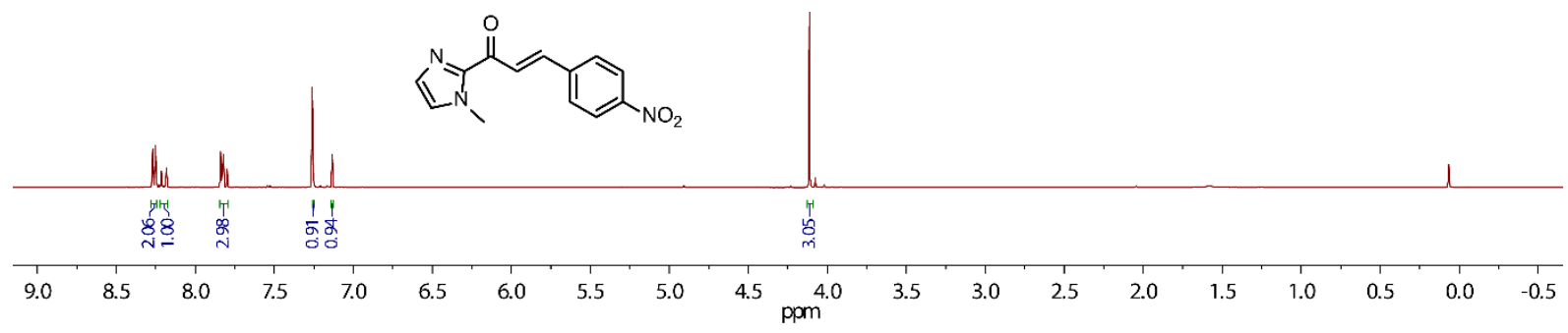

Figure S2. ${ }^{1} \mathrm{H}$ NMR $\left(500 \mathrm{MHz}, 298 \mathrm{~K}, \mathrm{CDCl}_{3}\right)$ of Michael-acceptor MA2.

\section{Michael-Acceptor MA3}<smiles>CC(=O)c1nccn1C</smiles><smiles>CC=Cc1ccc(OC)cc1</smiles><smiles>COc1ccc(/C=C/C(=O)c2nccn2C)cc1</smiles>

The synthesis of Michael-acceptor MA3 was the same procedure as for Michael-acceptor MA1 (52 mg, $0.21 \mathrm{mmol}$, $58 \%)$. The ${ }^{1} \mathrm{H}$ NMR was in accordance with literature. ${ }^{[5]}$

Chemical Formula: $\mathrm{C}_{14} \mathrm{H}_{14} \mathrm{~N}_{2} \mathrm{O}_{2}$

Molecular weight: $242.27 \mathrm{~g} \mathrm{~mol}^{-1}$

HRMS (ESI, pos., Calc. [M+H]+: 243.1128 ACN): Found: m/z=243.1123 [M+H]+, $243.1123[2 \mathrm{M}+\mathrm{H}]+$

${ }^{1}$ H NMR (500 MHz, $298 \mathrm{~K}$, DMSO-d $\left.{ }^{6}\right): \delta(\mathrm{ppm}): 7.92(\mathrm{~d}, J=15.9 \mathrm{~Hz}, 1 \mathrm{H}), 7.71(\mathrm{~d}, J=15.9 \mathrm{~Hz}, 1 \mathrm{H}), 7.73(\mathrm{~d}, J=8.7$ $\mathrm{Hz}, 2 \mathrm{H}), 7.58(\mathrm{~s}, 1 \mathrm{H}), 7.24(\mathrm{~s}, 1 \mathrm{H}), 7.02(\mathrm{~d}, J=8.4,2 \mathrm{H}), 4.00(\mathrm{~s}, 3 \mathrm{H}), 3.82(\mathrm{~s}, 3 \mathrm{H})$. 


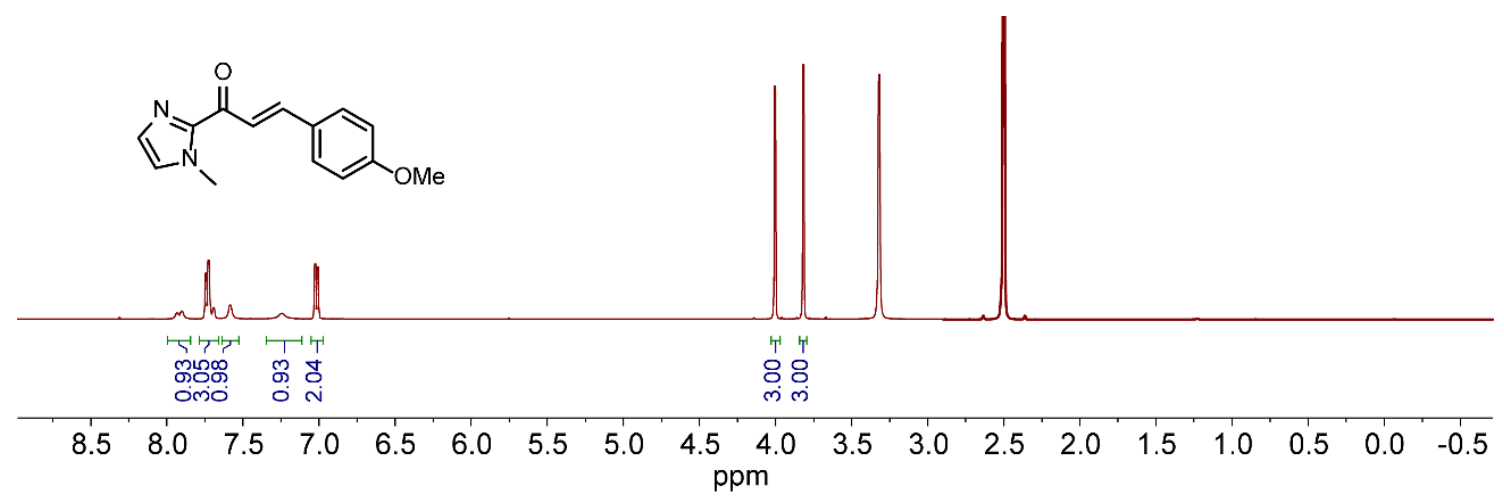

Figure S3. ${ }^{1} \mathrm{H}$ NMR $\left(500 \mathrm{MHz}, 298 \mathrm{~K}\right.$, DMSO-d $\left.{ }^{6}\right)$ of Michael-acceptor MA3.

\section{Michael-Acceptor MA4}

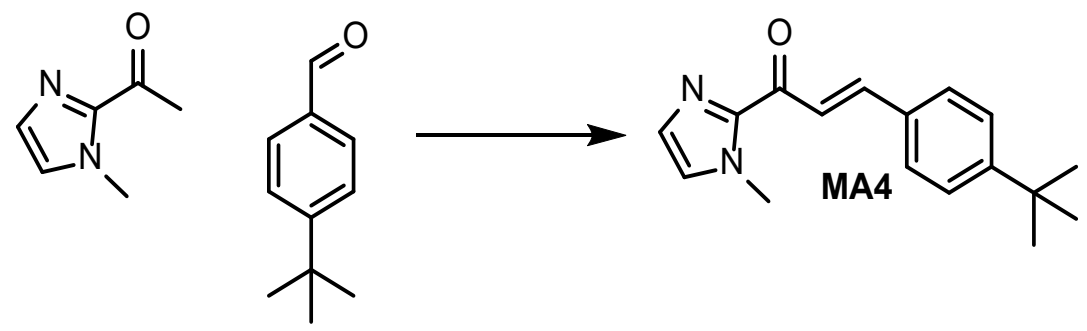

The synthesis of Michael-acceptor MA4 was the same procedure as for Michael-acceptor MA1 (81 mg, $0.30 \mathrm{mM}$, $82 \%)$.

Chemical Formula: $\mathrm{C}_{17} \mathrm{H}_{20} \mathrm{~N}_{2} \mathrm{O}$

Molecular weight: $268.36 \mathrm{~g} \mathrm{~mol}^{-1}$

HRMS (ESI, pos., Calc. [M+H]+: 269.1648 ACN): Found: m/z = 269.1648 [M+H]+, 537.3225 [2M+H].

${ }^{1} \mathbf{H}$ NMR $\left(600 \mathrm{MHz}, 298 \mathrm{~K}, \mathrm{CDCl}_{3}\right): \delta(\mathrm{ppm}): 8.05$ (d, $\left.J=16 \mathrm{~Hz}, 1 \mathrm{H}\right), 7.82(\mathrm{~d}, J=16 \mathrm{~Hz}, 1 \mathrm{H}), 7.65-7.62(\mathrm{~m}, 2 \mathrm{H})$, $7.43-7.40(\mathrm{~m}, 2 \mathrm{H}), 7.21(\mathrm{~s}, 1 \mathrm{H}), 7.06(\mathrm{~s}, 1 \mathrm{H}), 4.08(\mathrm{~s}, 3 \mathrm{H}), 1.33(\mathrm{~s}, 9 \mathrm{H})$.

${ }^{13}$ C NMR (151 MHz, $\left.298 \mathrm{~K}, \mathrm{CDCl}_{3}\right): \delta$ (ppm): 180.7, 154.2, 144.1, 143.5, 132.2, 129.3, 128.7, 127.2, 125.9, 121.9, $36.4,35.0,31.2$. 


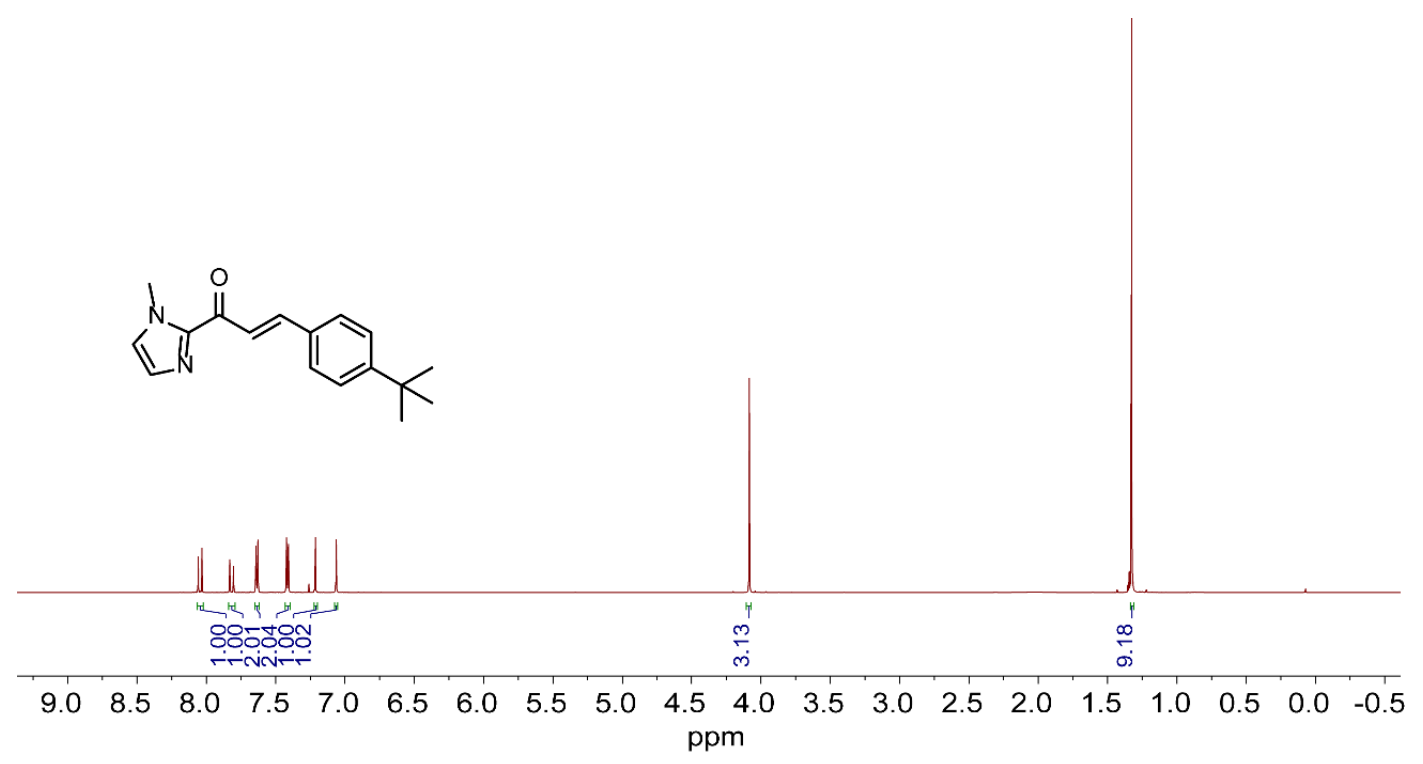

Figure S4. ${ }^{1} \mathrm{H}$ NMR $\left(600 \mathrm{MHz}, 298 \mathrm{~K}, \mathrm{CDCl}_{3}\right)$ of Michael-acceptor MA4.

\section{Michael-Acceptor MA5}<smiles>CC(=O)c1nccn1C</smiles><smiles>O=Cc1ccc(C(F)(F)F)cc1</smiles><smiles>Cn1ccnc1C(=O)/C=C/c1ccc(C(F)(F)F)cc1</smiles>

The synthesis of Michael-acceptor MA5 was the same procedure as for Michael-acceptor MA1 (80 mg, $0.29 \mathrm{mM}$, $78 \%){ }^{[4]}$

Chemical Formula: $\mathrm{C}_{14} \mathrm{H}_{11} \mathrm{~F}_{3} \mathrm{~N}_{2} \mathrm{O}$

Molecular weight: $280.25 \mathrm{~g} \mathrm{~mol}^{-1}$

HRMS (ESI, pos., Calc. [M+H]+: 281.0896 ACN): Found: m/z=281.0896 [M+H]+, 561.1722 [2M+H]+, 561.1722 .

${ }^{1} \mathbf{H}$ NMR $\left(600 \mathrm{MHz}, 298 \mathrm{~K}, \mathrm{CDCl}_{3}\right): \delta(\mathrm{ppm}): 8.16(\mathrm{~d}, J=16.0 \mathrm{~Hz}, 1 \mathrm{H}), 7.82(\mathrm{~d}, J=16.0 \mathrm{~Hz}, 1 \mathrm{H}), 7.8-7.78(\mathrm{~m}, 2 \mathrm{H})$, $7.67-7.64(\mathrm{~m}, 2 \mathrm{H}), 7.25(\mathrm{~s}, 1 \mathrm{H}), 7.11(\mathrm{~s}, 1 \mathrm{H}), 4.11(\mathrm{~s}, 3 \mathrm{H})$.

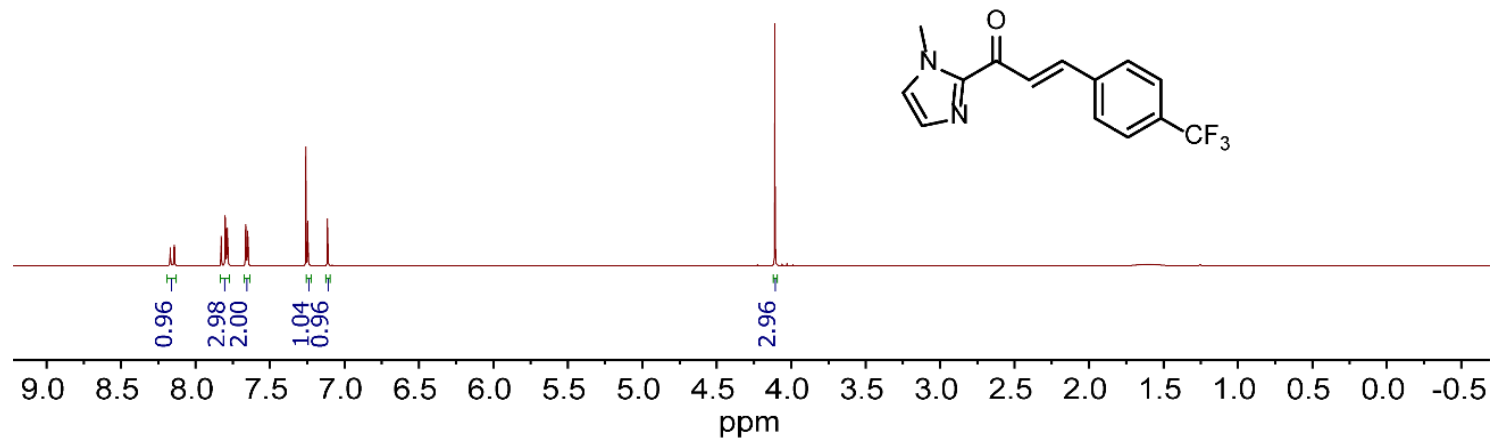

Figure S5. ${ }^{1} \mathrm{H}$ NMR $\left(600 \mathrm{MHz}, 298 \mathrm{~K}, \mathrm{CDCl}_{3}\right)$ of Michael-acceptor MA5. 


\section{Michael-Acceptor MA6}<smiles>CC(=O)c1nccn1C</smiles><smiles>Cc1ccc(C=O)cc1</smiles><smiles>Cc1ccc(/C=C/C(=O)c2nccn2C)cc1</smiles>

The synthesis of Michael-acceptor MA6 was the same procedure as for Michael-acceptor MA1 (60 mg, $0.27 \mathrm{mM}$, $72 \%)$. The ${ }^{1} \mathrm{H}$ NMR was in accordance with literature. ${ }^{[5]}$

Chemical Formula: $\mathrm{C}_{14} \mathrm{H}_{14} \mathrm{~N}_{2} \mathrm{O}$

Molecular weight: $226.28 \mathrm{~g} \mathrm{~mol}^{-1}$

HRMS (ESI, pos., Calc. [M+H]+: 227.1179 ACN): Found: m/z=227.1178 [M+H]+, 453.228 [2M+H]+.

${ }^{1}$ H NMR (500 MHz, $\left.298 \mathrm{~K}, \mathrm{CDCl}_{3}\right): \delta(\mathrm{ppm}): 8.04(\mathrm{~d}, J=16 \mathrm{~Hz}, 1 \mathrm{H}), 7.81(\mathrm{~d}, J=16 \mathrm{~Hz}, 1 \mathrm{H}), 7.62-7.58(\mathrm{~m}, 2 \mathrm{H})$, $7.22-7.19$ (m, 3H), $7.08(\mathrm{~s}, 1 \mathrm{H}), 4.09$ (s, 3H), 2.38 (s, 3H).

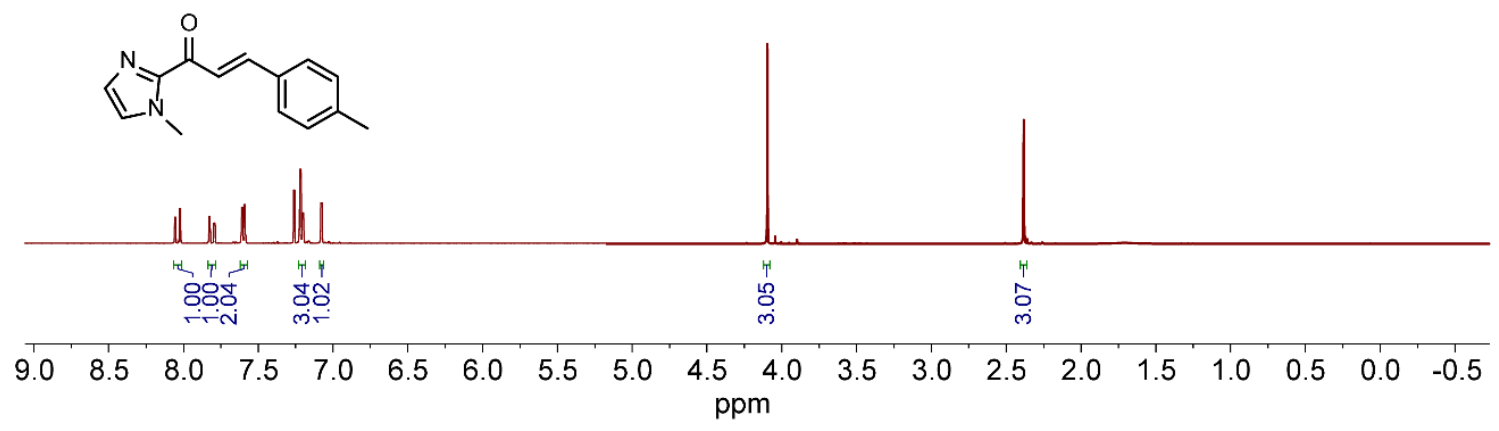

Figure S6. ${ }^{1} \mathrm{H}$ NMR $\left(500 \mathrm{MHz}, 298 \mathrm{~K}, \mathrm{CDCl}_{3}\right)$ of Michael-acceptor MA6.

\section{Michael-Acceptor MA7}<smiles>C[C+](C)c1cccc(C(=O)/C=C/c2ccccc2)n1</smiles>

The synthesis of Michael-acceptor MA7 was the same procedure as for Michael-acceptor MA1 (450 mg, $2.15 \mathrm{mM}$, $86 \%$ ). The ${ }^{1} \mathrm{H}$ NMR was in accordance with literature. ${ }^{[6]}$

Chemical Formula: $\mathrm{C}_{14} \mathrm{H}_{11} \mathrm{NO}$

Molecular weight: $209.25 \mathrm{~g} \mathrm{~mol}^{-1}$

HRMS (ESI, pos., Calc. [M+H]+: 210.0913, ACN): Found: m/z = 210.0912 [M+H]+, [2M+H]+.

${ }^{1}$ H NMR (700 MHz, $298 \mathrm{~K}, \mathrm{CDCl}_{3}$ ): $\delta$ (ppm): 8.77 (ddd, $\left.J=4.8,1.8,0.9 \mathrm{~Hz}, 1 \mathrm{H}\right), 8.34(\mathrm{~d}, J=16.0 \mathrm{~Hz}, 1 \mathrm{H}), 8.22$ (dt, $J=7.8,1.1 \mathrm{~Hz}, 1 \mathrm{H}), 7.96(\mathrm{~d}, J=16.0 \mathrm{~Hz}, 1 \mathrm{H}), 7.92(\mathrm{td}, J=7.7,1.7 \mathrm{~Hz}, 1 \mathrm{H}), 7.77-7.73(\mathrm{~m}, 2 \mathrm{H}), 7.52$ (ddd, $J=7.6$, $4.8,1.3 \mathrm{~Hz}, 1 \mathrm{H}), 7.46-7.39(\mathrm{~m}, 3 \mathrm{H})$. 

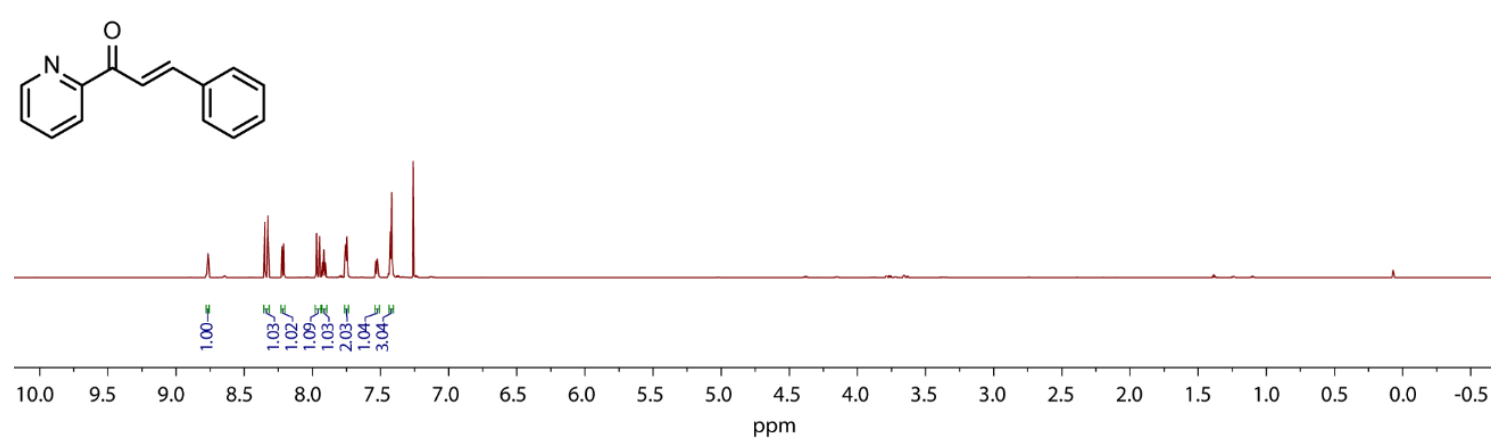

Figure S7. ${ }^{1} \mathrm{H}$ NMR $\left(700 \mathrm{MHz}, 298 \mathrm{~K}, \mathrm{CDCl}_{3}\right)$ of Michael-acceptor MA7.

\section{Michael-adduct A1}
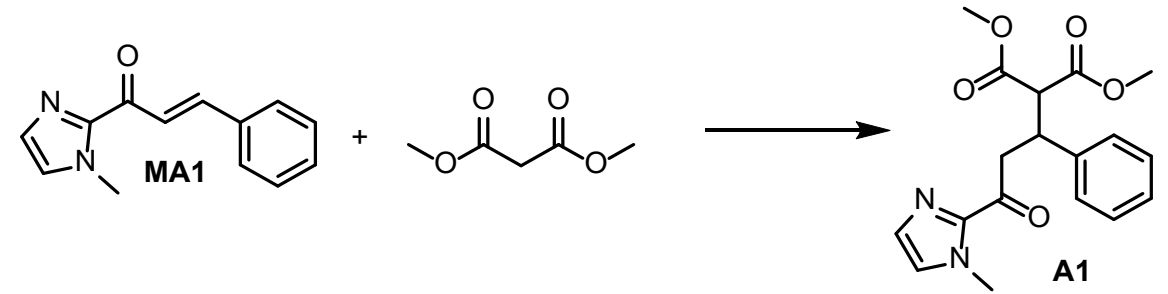

To a solution of dimethylmalonate (100 mg, $0.5 \mathrm{mmol}$, 1equiv.) and MA1 (62 mg, $0.5 \mathrm{mmol}$, 1equiv.) in methanol (5 $\mathrm{mL})$, sodium methoxide $(0.05 \mathrm{mmol}$, tip of a spatula) was added. The mixture was stirred at room temperature for 20

h. The solvent was removed and the remaining solid was purified by column chromatography $\left(\mathrm{CH}_{2} \mathrm{Cl}_{2} \rightarrow\right.$ $\left.\mathrm{CH}_{2} \mathrm{Cl}_{2} / \mathrm{MeOH}, 90 / 5\right)$ to afford the product as a white solid. (117 mg, $0.40 \mathrm{mM}, 85 \%$ ). The ${ }^{1} \mathrm{H}$ NMR was in accordance with literature. ${ }^{[3]}$

Chemical Formula: $\mathrm{C}_{18} \mathrm{H}_{20} \mathrm{~N}_{2} \mathrm{O}_{5}$

Molecular weight: $344.37 \mathrm{~g} \mathrm{~mol}^{-1}$

HRMS (ESI, pos., Calc. [M+H]+: 345.1445 ACN): Found: m/z = $313.1180[\mathrm{M}+\mathrm{H}-\mathrm{MeOH}]+345.1442[\mathrm{M}+\mathrm{H}]+$, $367.1261[\mathrm{M}+\mathrm{Na}]+$.

${ }^{1} \mathbf{H}$ NMR (500 MHz, $\left.298 \mathrm{~K}, \mathrm{CDCl}_{3}\right): \delta(\mathrm{ppm}): 7.31$ - $7.29(\mathrm{~m}, 2 \mathrm{H}), 7.26$ - $7.22(\mathrm{~m}, 2 \mathrm{H}), 7.18$ - $7.14(\mathrm{~m}, 1 \mathrm{H}), 7.10(\mathrm{~s}$, 1H), $6.95(\mathrm{~s}, 1 \mathrm{H}), 4.16\left(\mathrm{td},{ }^{3} \mathrm{~J}=10.0 \mathrm{~Hz}, 4.4 \mathrm{~Hz}, 1 \mathrm{H}\right), 3.87(\mathrm{~s}, 3 \mathrm{H}), 3.88-3.80(\mathrm{~m}, 2 \mathrm{H}), 3.72(\mathrm{~s}, 3 \mathrm{H}), 3.50-3.45(\mathrm{~m}$, $1 \mathrm{H}), 3.45(\mathrm{~s}, 3 \mathrm{H})$. 


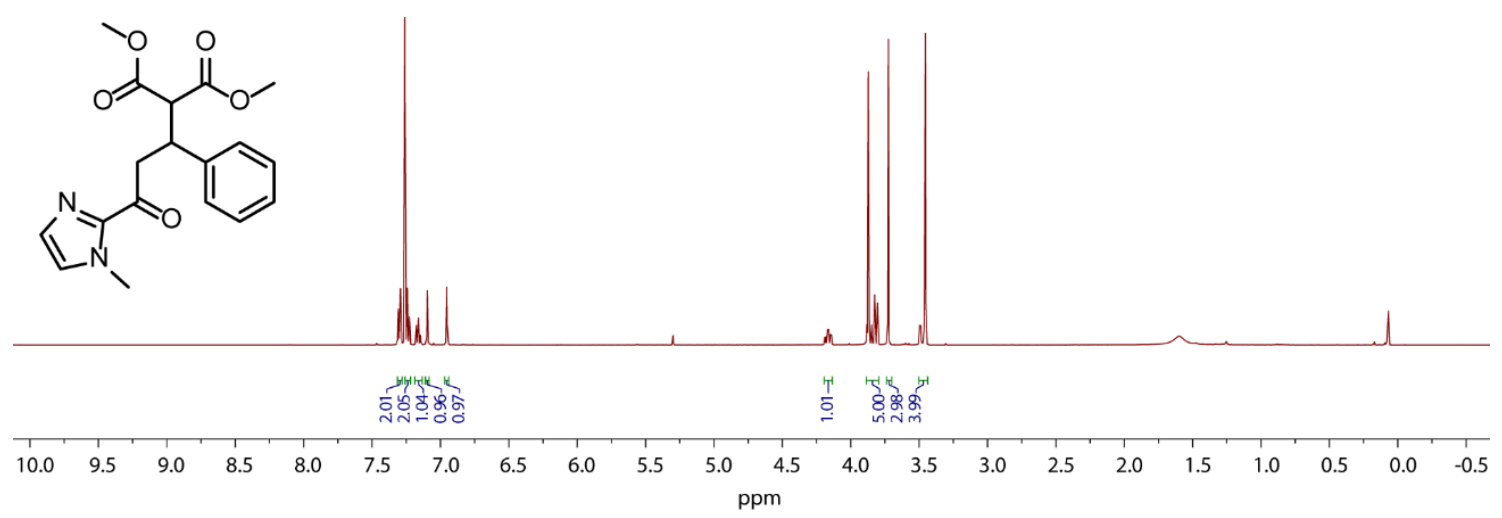

Figure S8. ${ }^{1} \mathrm{H}$ NMR (500 MHz, $\left.298 \mathrm{~K}, \mathrm{CDCl}_{3}\right)$ of Michael-adduct $\mathbf{A 1}$.

\section{Michael-adduct A2}

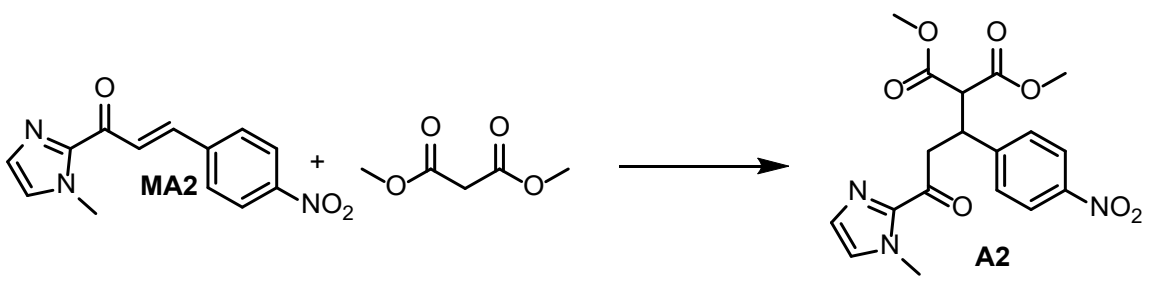

The synthesis of Michael-adduct A2 followed the general procedure described for Michael-adduct A1 (14 mg, $0.035 \mathrm{mM}, 45 \%)$.

Chemical Formula: $\mathrm{C}_{18} \mathrm{H}_{19} \mathrm{~N}_{3} \mathrm{O}_{7}$

Molecular weight: $389.36 \mathrm{~g} \mathrm{~mol}^{-1}$

HRMS (ESI, pos., Calc. [M+H]+: 390.1296, ACN): Found: m/z = 358.0310 [M+H-MeOH]+, 390.1293 [M+H]+.

${ }^{1}$ H NMR $\left(600 \mathrm{MHz}, 298 \mathrm{~K}, \mathrm{CDCl}_{3}\right): \delta(\mathrm{ppm}): 8.13-8.11(\mathrm{~m}, 2 \mathrm{H}), 7.53-7.50(\mathrm{~m}, 2 \mathrm{H}), 7.12(\mathrm{~s}, 1 \mathrm{H}), 6.99(\mathrm{~s}, 1 \mathrm{H})$, $4.27(\mathrm{td}, J=10.1,4.2 \mathrm{~Hz}, 1 \mathrm{H}), 3.94-3.83(\mathrm{~m}, 2 \mathrm{H}), 3.89(\mathrm{~s}, 3 \mathrm{H}), 3.76(\mathrm{~s}, 3 \mathrm{H}), 3.55-3.51(\mathrm{~m}, 1 \mathrm{H}), 3.50(\mathrm{~s}, 3 \mathrm{H})$.

${ }^{13}$ C NMR (151 MHz, $\left.298 \mathrm{~K}, \mathrm{CDCl}_{3}\right): \delta$ (ppm): 188.9, 168.3, 167.9, 148.5, 147.3, 142.4, 129.7, 129.0, 127.5, 123.9, $57.1,53.29,52.9,42.9,40.4,36.5$.

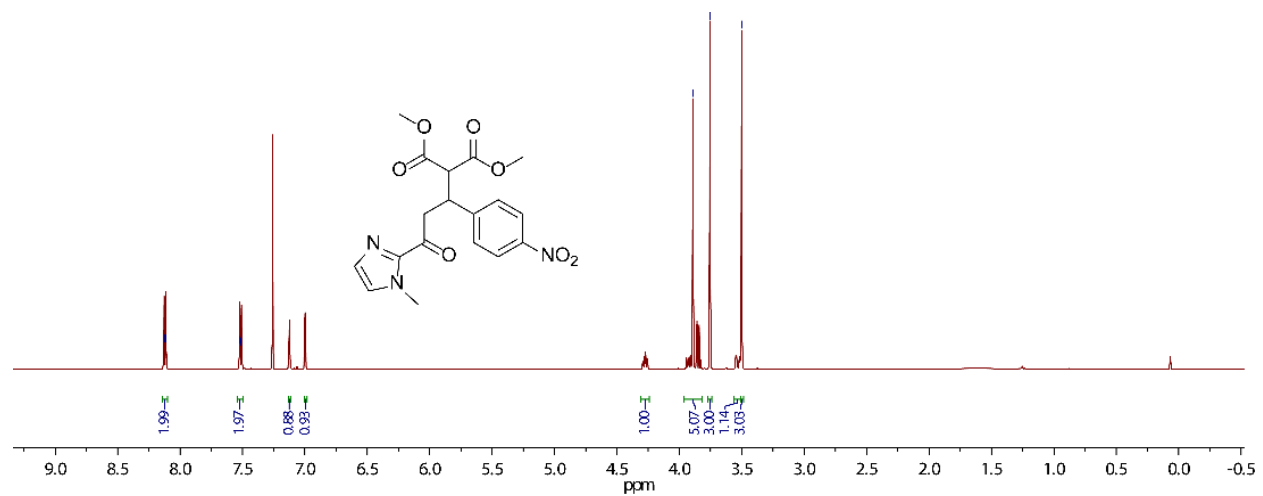

Figure S9. ${ }^{1} \mathrm{H}$ NMR $\left(600 \mathrm{MHz}, 298 \mathrm{~K}, \mathrm{CDCl}_{3}\right)$ of Michael-adduct $\mathbf{A 2}$. 

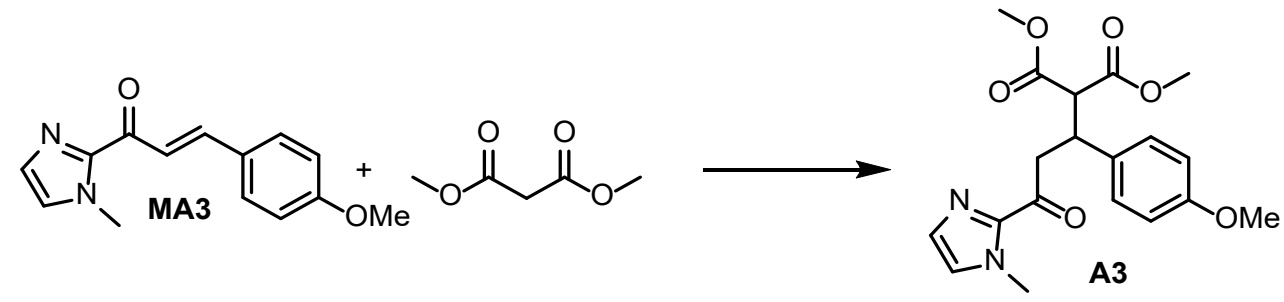

The synthesis of Michael-adduct A3 followed the general procedure described for Michael-adduct A1 (15 mg, $0.04 \mathrm{mM}, 48 \%$ ). The ${ }^{1} \mathrm{H}$ NMR was in accordance with literature. ${ }^{[3]}$

Chemical Formula: $\mathrm{C}_{19} \mathrm{H}_{22} \mathrm{~N}_{2} \mathrm{O}_{6}$

Molecular weight: $374.39 \mathrm{~g} \mathrm{~mol}^{-1}$

HRMS (ESI, pos., Calc. [M+H]+: 375.1551, ACN): Found: m/z=375.1547 [M+H]+, 397.1367 [M+Na]+.

${ }^{1}$ H NMR (500 MHz, $298 \mathrm{~K}$, DMSO-d $\left.{ }^{6}\right): \delta(\mathrm{ppm}): 7.42(\mathrm{~s}, 1 \mathrm{H}), 7.19-7.16(\mathrm{~m}, 2 \mathrm{H}), 7.07$ (s, 1H), $6.78-6.75(\mathrm{~m}, 2 \mathrm{H})$, $3.97(\mathrm{~d}, J=10.0 \mathrm{~Hz}, 1 \mathrm{H}), 3.90(\mathrm{td}, J=10.0,3.5 \mathrm{~Hz}, 1 \mathrm{H}), 3.84-3.78(\mathrm{~m}, 1 \mathrm{H}), 3.77$ (s, 3H), 3.67 (s, 3H), $3.63(\mathrm{~s}, 3 \mathrm{H})$, $3.39(\mathrm{~s}, 3 \mathrm{H}), 3.16(\mathrm{dd}, J=16.8,3.7 \mathrm{~Hz}, 1 \mathrm{H})$.

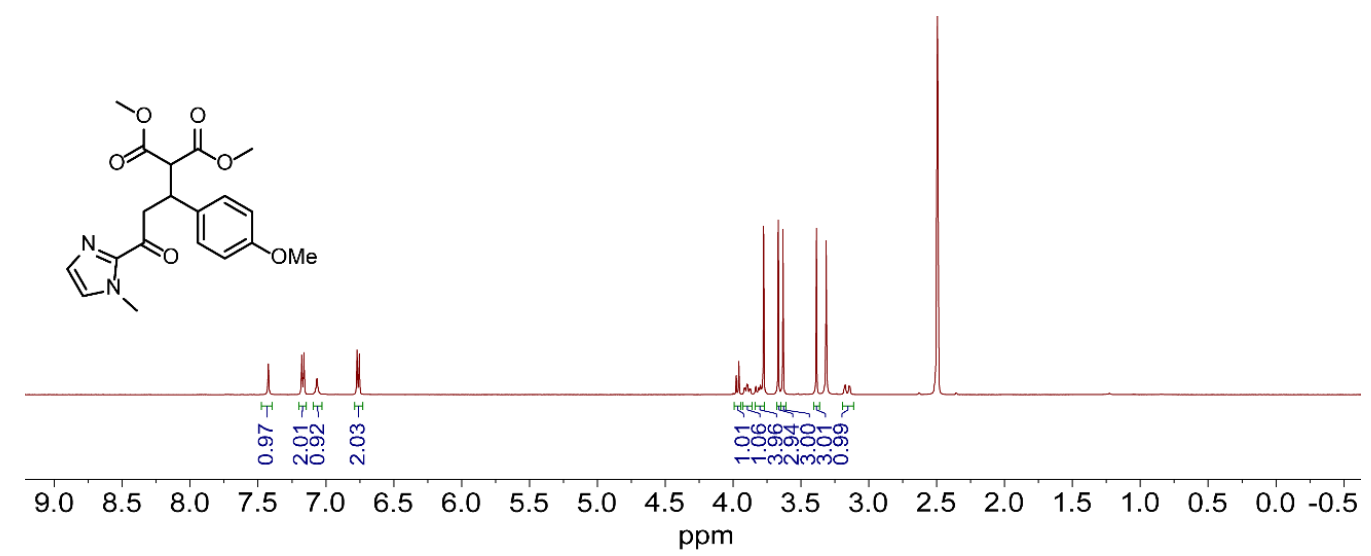

Figure S10. ${ }^{1} \mathrm{H}$ NMR $(500 \mathrm{MHz}, 298 \mathrm{~K}$, DMSO-d6) of Michael-adduct A3.

\section{Michael-adduct A4}
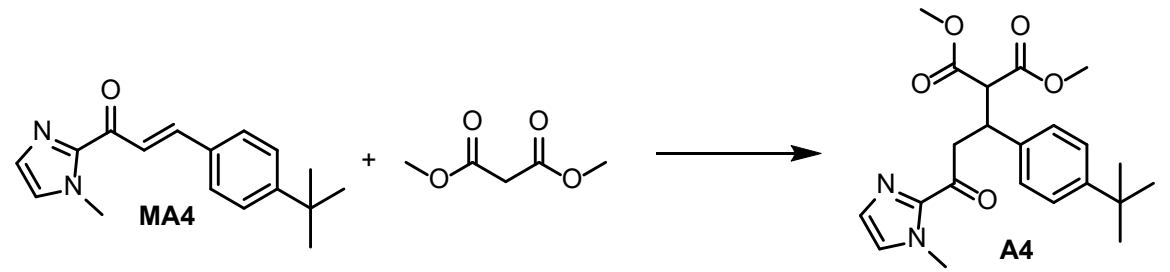

The synthesis of Michael-adduct A4 followed the general procedure described for Michael-adduct A1 (8 mg, $0.02 \mathrm{mM}$, $54 \%)$.

Chemical Formula: $\mathrm{C}_{22} \mathrm{H}_{28} \mathrm{~N}_{2} \mathrm{O}_{5}$

Molecular weight: $400.48 \mathrm{~g} \mathrm{~mol}^{-1}$ 
HRMS (ESI, pos., Calc. [M+H]+: 401.2071, ACN): Found: m/z = 401.2047 [M+H]+, 423.1865 [M+Na]+, 439.1603 $[\mathrm{M}+\mathrm{K}]+$.

${ }^{1}$ H NMR (600 MHz, $\left.298 \mathrm{~K}, \mathrm{CDCl}_{3}\right): \delta(\mathrm{ppm}):$ 7.26-7.21 (m, 4H), 7.18 (s, 1H), 6.99 (s, 1H), 4.13 (td, $J=10.4,9.2,4.5$ Hz, 1H), 3.91 (s, 3H), $3.94-3.82(\mathrm{~m}, 2 \mathrm{H}), 3.72(\mathrm{~s}, 3 \mathrm{H}), 3.59-3.53(\mathrm{~m}, 1 \mathrm{H}), 3.43$ (s, 3H), $1.25(\mathrm{~s}, 9 \mathrm{H})$.

${ }^{13}$ C NMR (151 MHz, $\left.298 \mathrm{~K}, \mathrm{CDCl}_{3}\right): \delta(\mathrm{ppm}): 168.6,168.2,149.8,137.0,127.5,127.7,126.4,125.2,125.2,57.4$, $52.6,52.2,43.5,39.9,36.4,34.3,31.2$.

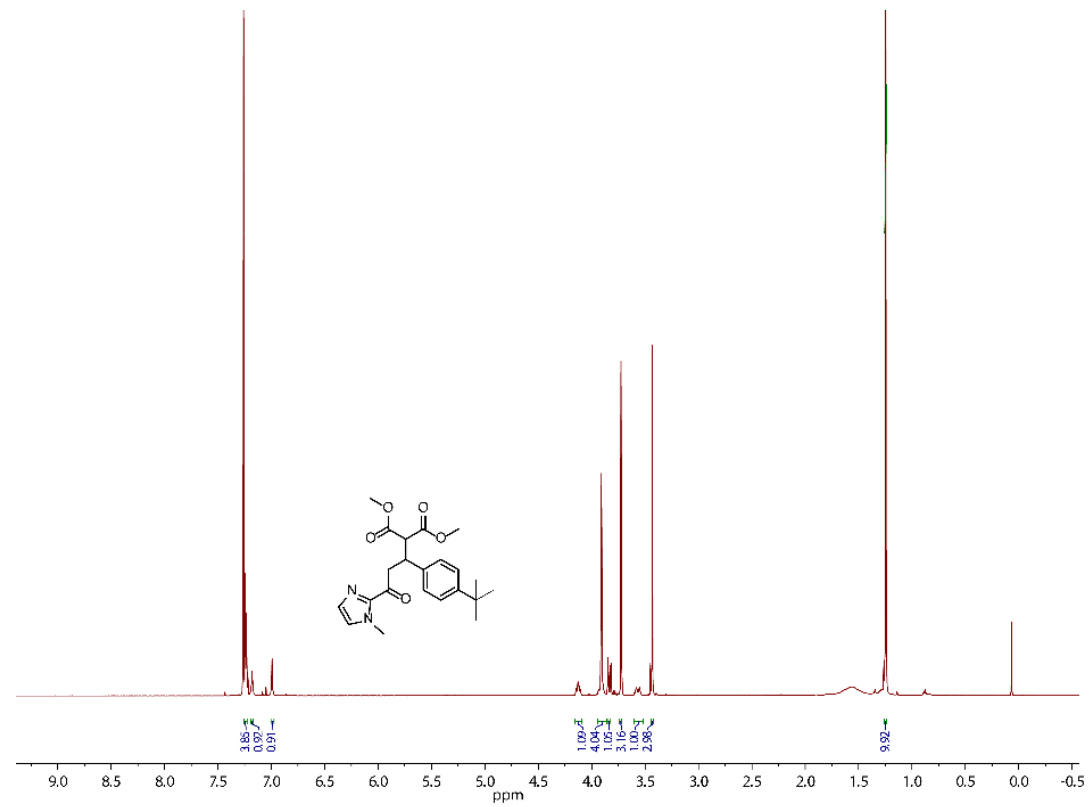

Figure S11. ${ }^{1} \mathrm{H}$ NMR $\left(00 \mathrm{MHz}, 298 \mathrm{~K}, \mathrm{CDCl}_{3}\right)$ of Michael-adduct $\mathbf{A 4}$.

\section{Michael-adduct A5}
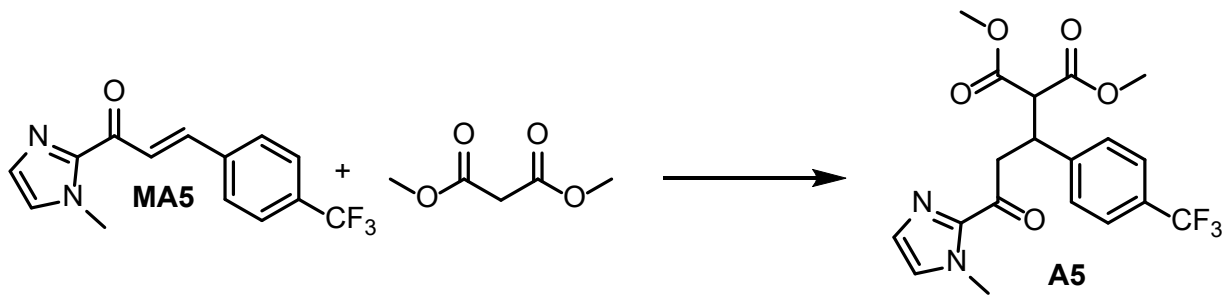

The synthesis of Michael-adduct A5 followed the general procedure described for Michael-adduct A1 (17.5 mg, $0.04 \mathrm{mM}, 59 \%)$.

\section{Chemical Formula: $\mathrm{C}_{19} \mathrm{H}_{19} \mathrm{~F}_{3} \mathrm{~N}_{2} \mathrm{O}_{5}$}

Molecular weight: $412.37 \mathrm{~g} \mathrm{~mol}^{-1}$

HRMS (ESI, pos., Calc. [M+H]+: 413.1319, ACN): Found: m/z = 413.1315 [M+H]+, 435.1134 [M+Na]+.

${ }^{1} \mathrm{H}$ NMR $\left(700 \mathrm{MHz}, 298 \mathrm{~K}, \mathrm{CDCl}_{3}\right): \delta(\mathrm{ppm}): 7.5$ (d, $\left.J=8.0 \mathrm{~Hz}, 2 \mathrm{H}\right), 7.44(\mathrm{~d}, J=8.0 \mathrm{~Hz}, 2 \mathrm{H}), 7.10(\mathrm{~s}, 1 \mathrm{H}), 6.97(\mathrm{~s}$, $1 \mathrm{H}), 4.23(\mathrm{td}, J=10.0,4.2 \mathrm{~Hz}, 1 \mathrm{H}), 3.89-3.82(\mathrm{~m}, 2 \mathrm{H}), 3.88(\mathrm{~s}, 3 \mathrm{H}), 3.74(\mathrm{~s}, 3 \mathrm{H}), 3.50-3.47(\mathrm{~m}, 1 \mathrm{H}), 3.47(\mathrm{~s}, 3 \mathrm{H})$. 
${ }^{13}$ C NMR (176 MHz, $\left.298 \mathrm{~K}, \mathrm{CDCl}_{3}\right): \delta(\mathrm{ppm}): 189.0,168.1,167.7,144.7,142.5,129.3$ (q, $\left.J=32.3 \mathrm{~Hz}\right), 128.9$, 128.8, 127.0, 125.3 (q, $J=3.8 \mathrm{~Hz}), 124.1$ (q, $J=272.0 \mathrm{~Hz}), 57.09,52.80,52.45,42.58,40.06,36.07$.

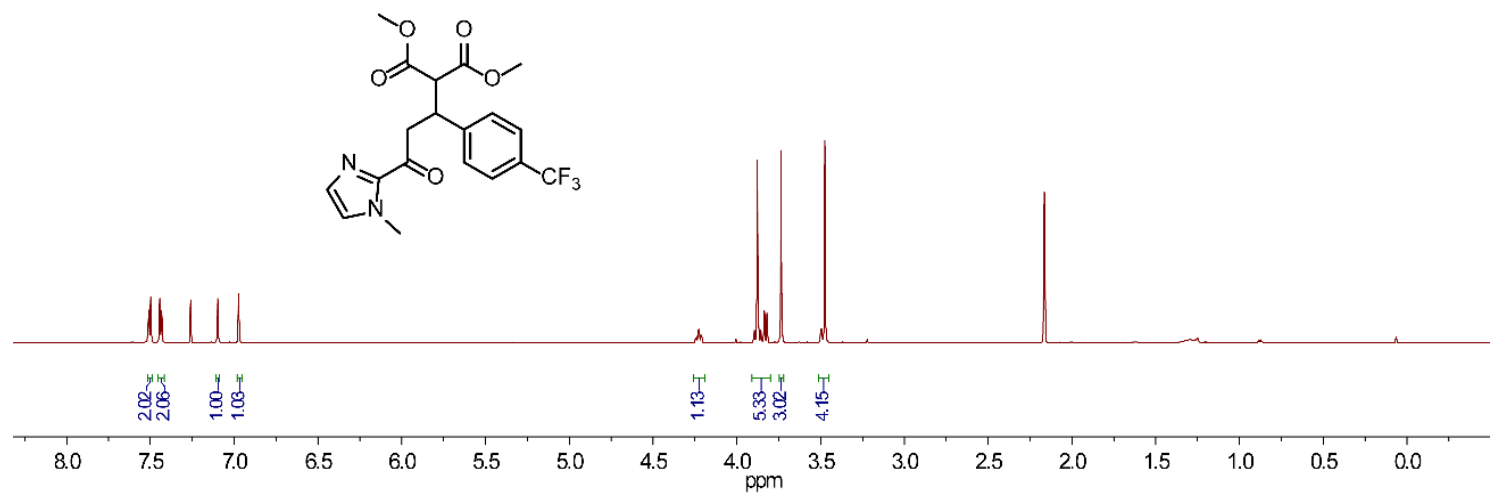

Figure S12. ${ }^{1} \mathrm{H}$ NMR $\left(700 \mathrm{MHz}, 298 \mathrm{~K}, \mathrm{CDCl}_{3}\right)$ of Michael-adduct $\mathbf{A 5}$.

\section{Michael-adduct A6}
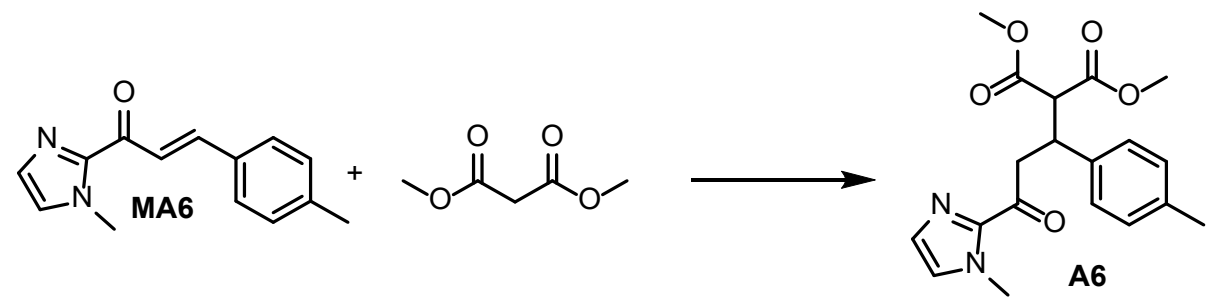

The synthesis of Michael-adduct A6 followed the general procedure described for Michael-adduct A1 (17.5 mg, $0.06 \mathrm{mM}, 73 \%)$.

Chemical Formula: $\mathrm{C}_{19} \mathrm{H}_{22} \mathrm{~N}_{2} \mathrm{O}_{5}$

Molecular weight: $358.39 \mathrm{~g} \mathrm{~mol}^{-1}$

HRMS (ESI, pos., Calc. [M+H]+: 359.1601, ACN): Found: m/z=359.1597 [M+H]+, 381.1416 [M+Na]+.

${ }^{1}$ H NMR (700 MHz, $\left.298 \mathrm{~K}, \mathrm{CDCl}_{3}\right): \delta(\mathrm{ppm}): 7.18$ (d, $\left.J=8.0 \mathrm{~Hz}, 2 \mathrm{H}\right), 7.12(\mathrm{~s}, 1 \mathrm{H}), 7.04(\mathrm{~d}, J=8.0 \mathrm{~Hz}, 2 \mathrm{H}), 6.95$ (s, $1 \mathrm{H}), 4.16-4.08(\mathrm{td}, J=10,4.3 \mathrm{~Hz}, 1 \mathrm{H}), 3.86(\mathrm{~s}, 3 \mathrm{H}), 3.85-3.78(\mathrm{~m}, 2 \mathrm{H}), 3.71(\mathrm{~s}, 3 \mathrm{H}), 3.51-3.46(\mathrm{~m}, 1 \mathrm{H}), 3.47$ (s, $3 \mathrm{H}), 2.25(\mathrm{~s}, 3 \mathrm{H})$.

${ }^{13}$ C NMR (176 MHz, $\left.298 \mathrm{~K}, \mathrm{CDCl}_{3}\right): \delta$ (ppm): 189.6, 168.5, 168.1, 142.7, 137.4, 136.5, 129.0, 128.7, 128.0, 126.7, 57.7, 52.6, 52.3, 42.8, 39.9, 36.0, 21.0. 


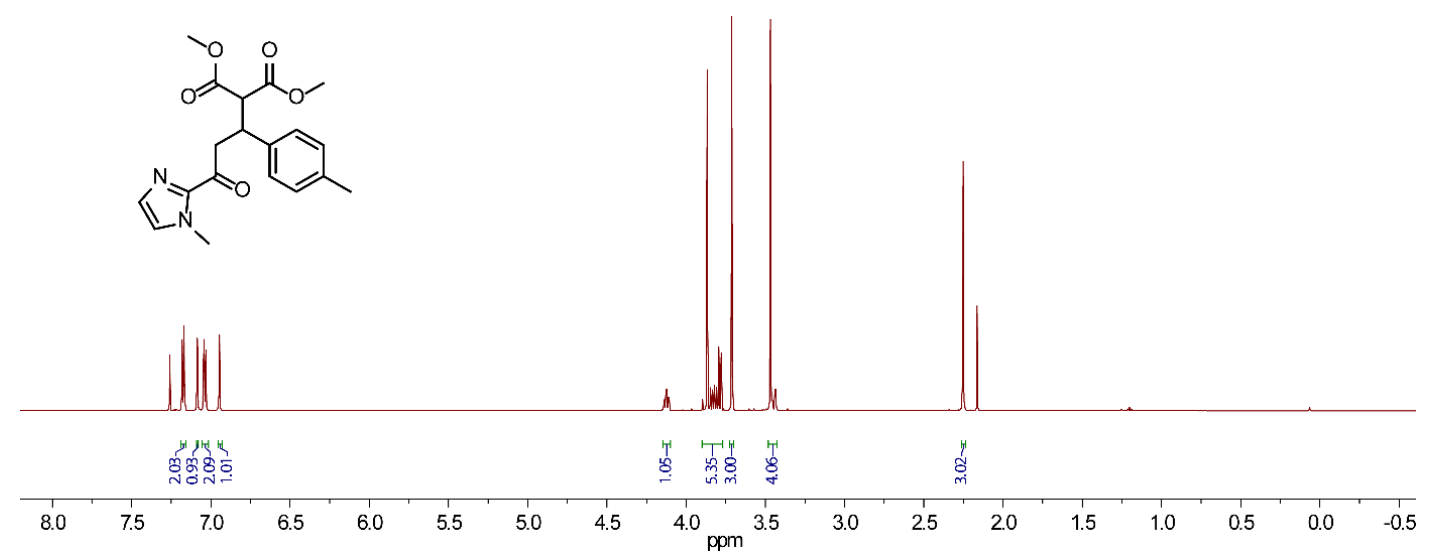

Figure S13. ${ }^{1} \mathrm{H}$ NMR $\left(700 \mathrm{MHz}, 298 \mathrm{~K}, \mathrm{CDCl}_{3}\right)$ of Michael-adduct $\mathbf{A 6}$.

\section{Michael-adduct A7}<smiles>O=C(/C=C/c1ccccc1)c1ccccn1</smiles><smiles>COC(=O)CC(=O)OC</smiles>
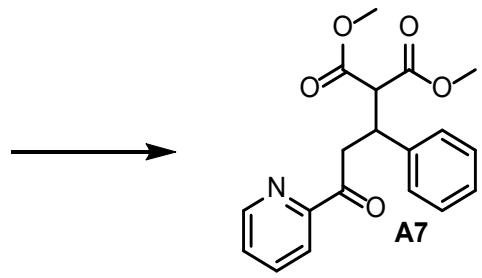

The synthesis of Michael-adduct A7 followed the general procedure described for Michael-adduct A1 (101 mg, $0.27 \mathrm{mM}, 55 \%)$.

Chemical Formula: $\mathrm{C}_{19} \mathrm{H}_{19} \mathrm{NO}_{5}$

Molecular weight: $341.36 \mathrm{~g} \mathrm{~mol}^{-1}$

HRMS (ESI, pos., Calc. [M+H]+: 342.1336 ACN): Found: m/z=342.1322 [M+H]+, $364.1155[\mathrm{M}+\mathrm{Na}]+$.

${ }^{1} \mathbf{H}$ NMR $\left(700 \mathrm{MHz}, 298 \mathrm{~K}, \mathrm{CDCl}_{3}\right): \delta(\mathrm{ppm}): 8.65(\mathrm{~d}, J=4.7 \mathrm{~Hz}, 1 \mathrm{H}), 7.91(\mathrm{~d}, J=7.8 \mathrm{~Hz}, 1 \mathrm{H}), 7.76(\mathrm{td}, J=7.7,1.7$ $\mathrm{Hz}, 1 \mathrm{H}), 7.46-7.39(\mathrm{~m}, 1 \mathrm{H}), 7.33-7.29(\mathrm{~m}, 2 \mathrm{H}), 7.26-7.22(\mathrm{~m}, 2 \mathrm{H}), 7.17-7.13(\mathrm{~m}, 1 \mathrm{H}), 4.22(\mathrm{td}, J=9.7,4.3 \mathrm{~Hz}$, $1 \mathrm{H}), 3.93(\mathrm{dd}, J=17.9,9.3 \mathrm{~Hz}, 1 \mathrm{H}), 3.86(\mathrm{~d}, J=10.2 \mathrm{~Hz}, 1 \mathrm{H}), 3.72(\mathrm{~s}, 3 \mathrm{H}), 3.60(\mathrm{dd}, J=18.0,4.5 \mathrm{~Hz}, 1 \mathrm{H}), 3.47(\mathrm{~s}$, $3 \mathrm{H})$.

${ }^{13}$ C NMR (151 MHz, $\left.298 \mathrm{~K}, \mathrm{CDCl}_{3}\right): \delta$ (ppm):199.1, 168.9, 168.5, 153.4, 149.1, 141.1, 137.2, 128.6, 128.5, 127.4, $127.3,122.1,57.9,52.9,52.6,42.0,40.7$. 


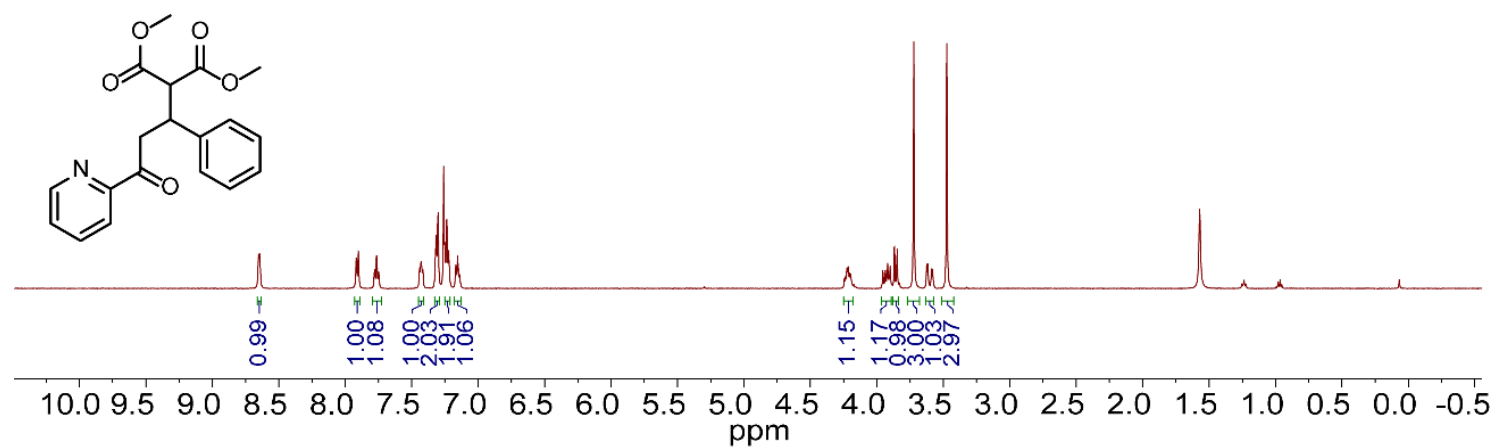

Figure S14. ${ }^{1} \mathrm{H}$ NMR $\left(500 \mathrm{MHz}, 298 \mathrm{~K}, \mathrm{CDCl}_{3}\right)$ of Michael-acceptor A7.

\section{Oligonucleotide synthesis and purification}

All oligonucleotides were synthesized on a $K \& A$ Laborgeraete $G b R H-8$ synthesizer on a $1 \mu$ mol scale using the standard phosphoramidite methods on CPG and following previously published synthetic procedures. ${ }^{[7,8]}$

Table S1: Reagents for DNA synthesis.

\begin{tabular}{lll}
\hline name & reagent & composition \\
\hline DCA & detritylation & $3 \%(\mathrm{v} / \mathrm{v})$ dichloroacetic acid in anhydrous dichloromethane \\
ACT & activator & $0.3 \mathrm{M} 5$-(benzylthio)-1H-tetrazole in anhydrous acetonitrile \\
Cap A & capping A & $10 \%(\mathrm{v} / \mathrm{v}) \mathrm{N}$-methyl imidazole in anhydrous tetrahydrofurane \\
Cap B & capping B & $2,6-$ lutidine / acetic anhydride / anhydrous tetrahydrofurane $1: 1: 8(\mathrm{v} / \mathrm{v} / \mathrm{v})$ \\
OXI & Oxidizer & $0.02 \mathrm{M}$ iodine in tetrahydrofurane / pyridine / water 7:2:1 (v/v/v) \\
ACN & & anhydrous acetonitrile \\
\hline
\end{tabular}

Standard phosphoramidites (DMT-dT-CEP, DMT-dA(Bz)-CEP and DMT-dG $(i \mathrm{Bu})-\mathrm{CEP})$ were used and the cartridges were manually packed with controlled pore glass (CPG) solid supports (1000, , 25-35 $\mu \mathrm{mol} / \mathrm{g}, \mathrm{DMT}-\mathrm{dG}(i \mathrm{Bu})-\mathrm{CPG})$. The oligonucleotide synthesis followed the built-in methods of the DNA synthesizer and was slightly modified. First, the cartridges were treated three times with DCA to deprotect the 5'-OH groups. Second, coupling was achieved by mixing the respective phosphoramidite $(0.1 \mathrm{M}$ in $\mathrm{ACN})$ with $\mathrm{ACT}(1: 1, \mathrm{v} / \mathrm{v})$. The coupling time was $\sim 0.5 \mathrm{~min}$ for standard phosphoramidites and $\sim 3.5 \mathrm{~min}$ for the ligand-modified phosphoramidites. Third, the cartridge was treated with a 1:1 (v/v) mixture of Cap A and Cap B to acetylate unreacted 5'-OH groups, which was followed, by the oxidation with OXI. Here, an additional washing step with ACN was introduced compared to the standard routine. After each individual step of the cycle, the cartridge was washed with ACN followed by a drying step with argon. The described cycle was repeated for every incorporated nucleotide.

After DNA synthesis, the solid supports were removed from the cartridges and treated with $0.5 \mathrm{~mL}$ of concentrated aqueous $\mathrm{NH}_{4} \mathrm{OH}$ at $55^{\circ} \mathrm{C}$ overnight for cleavage from the $\mathrm{CPG}$ and for deprotection. The supernatant solution was filtered (VWR Centrifugal filters) and the CPG was washed with $100 \mu \mathrm{L} \mathrm{H}_{2} \mathrm{O}$. The volume was reduced under reduced pressure using a $H$. Saur Laborbedarf S-Concentrator BA-VC-300H vacuum concentrator to $\sim 0.3 \mathrm{~mL}$. Oligonucleotides were purified by reversed-phase HPLC on an Agilent Technologies 1260 Infinity II HPLC system equipped with an autosampler, column oven, DAD detector and a Macherey-Nagel VP 250/10 NUCLEODUR 100-5 
C18ec column (oven temperature: $60{ }^{\circ} \mathrm{C}$, flow rate: $2.5 \mathrm{~mL} / \mathrm{min}$, solvent A: $50 \mathrm{mM}$ TEAA pH 7, solvent B: $70: 30$ $\mathrm{MeCN} / 50$ mM TEAA pH 7, gradient: from 100\% solvent A to $20 \%$ solvent A and $80 \%$ solvent B in 30 min).

Subsequently, the cleavage of the 5'-OH DMT protecting groups (with 2\% TFA) and desalting were accomplished using Waters Sep-Pak C18 cartridges. Desalted oligonucleotides were lyophilized using a Christ Alpha 2-4 LSCbasic lyophilisation device and stored as a solid or as $0.5-1 \mathrm{mM}$ stock solution in water at $-20{ }^{\circ} \mathrm{C}$.

The concentrations of all oligonucleotide stock solutions were determined via the absorbance at $260 \mathrm{~nm}$ at $25^{\circ} \mathrm{C}$ with a Thermo Scientific Nanodrop One instrument and using extinction coefficients for the nucleosides.

Table S2. Investigated sequences.

\begin{tabular}{|c|c|}
\hline Name & Sequence $5^{\prime} \rightarrow 3^{\prime}$ \\
\hline htel $\mathbf{L}_{2} \mathrm{~A}$ & AGG GTL TGG GTT AGG GLT TGG G \\
\hline htell $\mathbf{L}_{2} \mathrm{~B}$ & AGG GTL TGG GTT AGG GTL TGG G \\
\hline htel $\mathbf{L}_{2} \mathrm{C}$ & AGG GTT LGG GTT AGG GTL TGG G \\
\hline htel $\mathbf{L}_{2} \mathrm{D}$ & AGG GLT TGG GTT AGG GTL TGG G \\
\hline htell $\mathbf{L}_{2} \mathrm{E}$ & AGG GTL TGG GTT AGG GTT LGG G \\
\hline htell $\mathbf{L}_{2} \mathrm{~F}$ & AGG GLL TGG GTT AGG GTT AGG G \\
\hline htell $\mathbf{L}_{2} \mathrm{G}$ & AGG GTL LGG GTT AGG GTT AGG G \\
\hline htel $\mathbf{L}_{2} \mathrm{H}$ & AGG GLT LGG GTT AGG GTT AGG G \\
\hline htell $\mathbf{L}_{2} \mathrm{I}$ & AGG GTT AGG GTT AGG GTL LGG G \\
\hline htell $\mathbf{L}_{2} \mathbf{J}$ & AGG GTT AGG GTT AGG GLL TGG G \\
\hline htel $\mathbf{L}_{2} \mathrm{~K}$ & AGG GTL AGG GTT AGG GTL TGG G \\
\hline htel $\mathbf{L}_{2} \mathrm{~L}$ & AGG TTA LGG TTA GGL TTA GG \\
\hline htell $\mathbf{L}_{3} \mathrm{~A}$ & AGG GLT LGG GTT AGG GTL TGG G \\
\hline htell $\mathbf{L}_{3} \mathrm{~B}$ & AGG GTL TGG GTT AGG GLT LGG G \\
\hline htel $\mathbf{L}_{3} \mathrm{C}$ & AGG GTL TGG GTT AGG GLL TGG G \\
\hline htell $\mathbf{L}_{3} \mathrm{D}$ & AGG GTL TGG GTT AGG GTL LGG G \\
\hline htell $\mathbf{L}_{3} \mathrm{E}$ & AGG GLL TGG GTT AGG GTL TGG G \\
\hline htell $\mathbf{L}_{3} \mathrm{~F}$ & AGG GTL LGG GTT AGG GTL TGG G \\
\hline htell $\mathbf{L}_{3} \mathrm{G}$ & AGG GTT LGG GTT AGG GLL TGG G \\
\hline htel $\mathbf{L}_{3} \mathrm{H}$ & AGG GTL AGG GTT AGG GLT LGG G \\
\hline htell $\mathbf{L}_{3} \mathrm{I}$ & AGG GAL TGG GTT AGG GLT LGG G \\
\hline htelL $\mathbf{L}_{3} \mathbf{J}$ & AGG GTL TGG GTT AGG GLA LGG G \\
\hline htel $\mathbf{L}_{3} \mathrm{~K}$ & AGG GTL AGG GTT AGG GLT LGG G \\
\hline htell $\mathbf{L}_{3} \mathrm{~L}$ & AGG GAL TGG GTT AGG GLT LGG G \\
\hline htel. $\mathbf{L}_{3} \mathrm{M}$ & AGG GTL TGG GTT AGG GLA LGG G \\
\hline htell $\mathbf{L}_{3} \mathrm{~N}$ & AGG LTT ALG GTT AGG LTT AGG \\
\hline htel $\mathbf{L}_{3} \mathrm{O}$ & AGG LTT ALG GTT AGG TTA LGG \\
\hline htell $\mathbf{L}_{4} \mathrm{~A}$ & AGG LTT ALG GTT AGG LTT ALG G \\
\hline htelL $4 \mathrm{~B}$ & AGG TLT LGG TTA GGL TLA GG \\
\hline htell $\mathbf{L}_{4} \mathrm{C}$ & AGG TLT LGG TTA GGT LTL GG \\
\hline htel. 6 & AGG LTL TLG GTT AGG LTL TLG G \\
\hline htel. $\mathbf{L}_{7}$ & AGG LTL LTL GGT TAG GLT LTL GG \\
\hline htel 22 & AGG GTT AGG GTT AGG GTT AGG G \\
\hline c-myc & TGG GAG GGT GGG GAG GGT GGG GAAG G \\
\hline $91 \mathbf{L}_{3}$ & GTG LGT GTG AGL GTG LGT GAG G \\
\hline $92 \mathbf{L}_{2}$ & GTG AGT GTG AGL GTG LGT GAG G \\
\hline
\end{tabular}




\section{Michael-Addition}

Samples were prepared at concentrations of $120 \mu \mathrm{M}$ G-quadruplex, $100 \mu \mathrm{M} \mathrm{CuSO}_{4}, 10 \mathrm{mM}$ HEPES pH 8 and 100 $\mathrm{mM} \mathrm{KCl} / \mathrm{NaCl}$ at a total volume of $75 \mu \mathrm{L}$. To form the G-quadruplexes, solutions were heated to $85^{\circ} \mathrm{C}$ and cooled down to $4{ }^{\circ} \mathrm{C}$ at $0.5^{\circ} \mathrm{C} \mathrm{min}^{-1}$. To the G-quadruplex solutions, Michael-acceptor 1 ( $0.75 \mathrm{uL}$ of a $100 \mathrm{mM}$ DMSO stock solution) and DMM (Dimethylmalonate) were added followed by rigorous mixing and three days incubation in a $V W R$ Cooling Thermal Shake Touch $\left(5{ }^{\circ} \mathrm{C}, 600 \mathrm{rpm}\right)$. After three days samples were extracted with $\mathrm{Et}_{2} \mathrm{O}(150 \mu \mathrm{L})$, the organic phase was dried over $\mathrm{MgSO}_{4}$ and the solvent removed in vacuum concentrators (H.Saur S-Concentrator BA$V C-300 H)$. To determine conversion and enantiomeric excess, samples were dissolved in $n$ Hexane/isoPronanol (70/30) and analysed by chiral HPLC. To determine the absolute stereo configuration of the Michael-adduct the optical activity $[\alpha]$ of enantiopure product was measured. Comparison with literature led to the conclusion that the enantiomer eluting at $\sim 14$ min was the $(S)$ enantiomer and at $\sim 20 \min$ the $(R)$ enantiomer. ${ }^{[9]}$
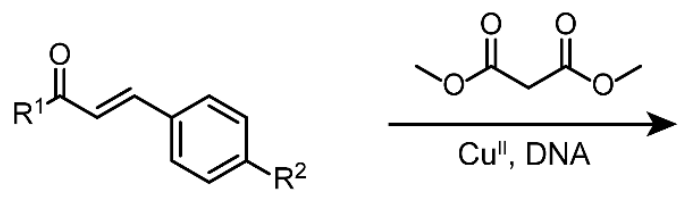<smiles>[Z][Z]1nccn1C</smiles>

$\mathrm{N}$-methyl imidazole<smiles>[Y]=[X]1ccccn1</smiles>

pyridine<smiles>[R]C(=O)CC(=C1C=CC([R2])=CC1)C(C(=O)OC)C(=O)OC</smiles>

$$
\begin{aligned}
& \text { MA1 }=\mathrm{R}^{1}=\mathrm{N} \text {-methyl imidazole, } \mathrm{R}^{2}=\mathrm{H} \\
& \text { MA2 }=\mathrm{R}^{1}=\mathrm{N} \text {-methyl imidazole, } \mathrm{R}^{2}=-\mathrm{NO}_{2} \\
& \text { MA3 }=\mathrm{R}^{1}=\mathrm{N} \text {-methyl imidazole, } \mathrm{R}^{2}=-\mathrm{OCH}_{3} \\
& \text { MA4 }=\mathrm{R}^{1}=\mathrm{N} \text {-methyl imidazole, } \mathrm{R}^{2}=-\mathrm{tbutyl} \\
& \text { MA5 }=\mathrm{R}^{1}=\mathrm{N} \text {-methyl imidazole, } \mathrm{R}^{2}=-\mathrm{CF}_{3} \\
& \text { MA6 }=\mathrm{R}^{1}=\mathrm{N} \text {-methyl imidazole, } \mathrm{R}^{2}=-\mathrm{CH}_{3} \\
& \text { MA7 }=\mathrm{R}^{1}=\text { pyridine, } \mathrm{R}^{2}=\mathrm{H}
\end{aligned}
$$

Figure S15. Investigated substrate scope.

\section{Chiral HPLC}

Conversion and enantiomeric excess (ee) were determined using an Agilent Technologies 1260 Infinity HPLC system equipped with a normal phase CHIRALPAK IA-3 column (Amylose tris(3,5-dimethylphenylcarbamate) immobilized on $3 \mu \mathrm{m}$ silica-gel) and a CHIRALPAK IC-3 guard column (Cellulose tris(3,5-dichlorophenylcarbamate) immobilized on $3 \mu \mathrm{m}$ silica-gel) using a solvent mixture of $n$ Hexane/isoPropanol, $80 / 20$ with a flow rate of $1 \mathrm{~mL} \mathrm{~min}^{-1}$. The signals at $250 \mathrm{~nm}$ were integrated to calculate conversion and $e e$. The detector bandwidth was set to $8 \mathrm{~nm}$. The detection limit for the enantioselectivity was $\sim 99 \%$ ee.

The signals of starting material and product were calibrated by injecting equal volumes of $1 \mathrm{mM}$ stock solutions of the Michael-acceptor and Michael-adduct (compare Fig. 1). For substrates MA2, MA5 and MA7 significant differences to the product absorption were observed and correction factors of $0.36,0.68,2.88$, respectively, were introduced to correct the conversions monitored by peak integration in the HPLC traces. 

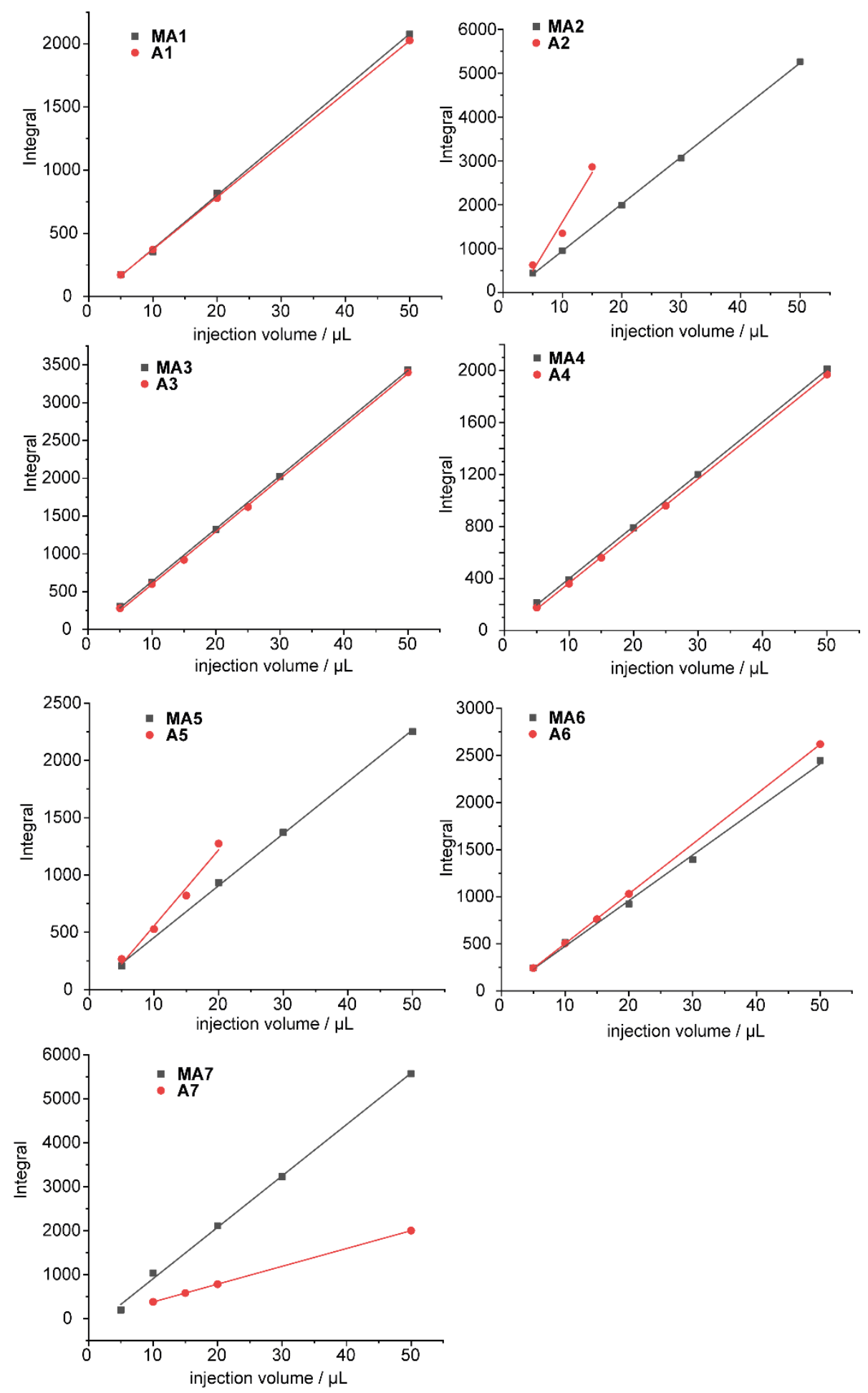

Figure S16: HPLC calibration of MA1-7 (black) and A1-7 (red). Varying volumes of $1 \mathrm{mM}$ stock solutions (nHexane/isopropanol, 70/30) were injected and the corresponding signals at $250 \mathrm{~nm}$ were integrated. 

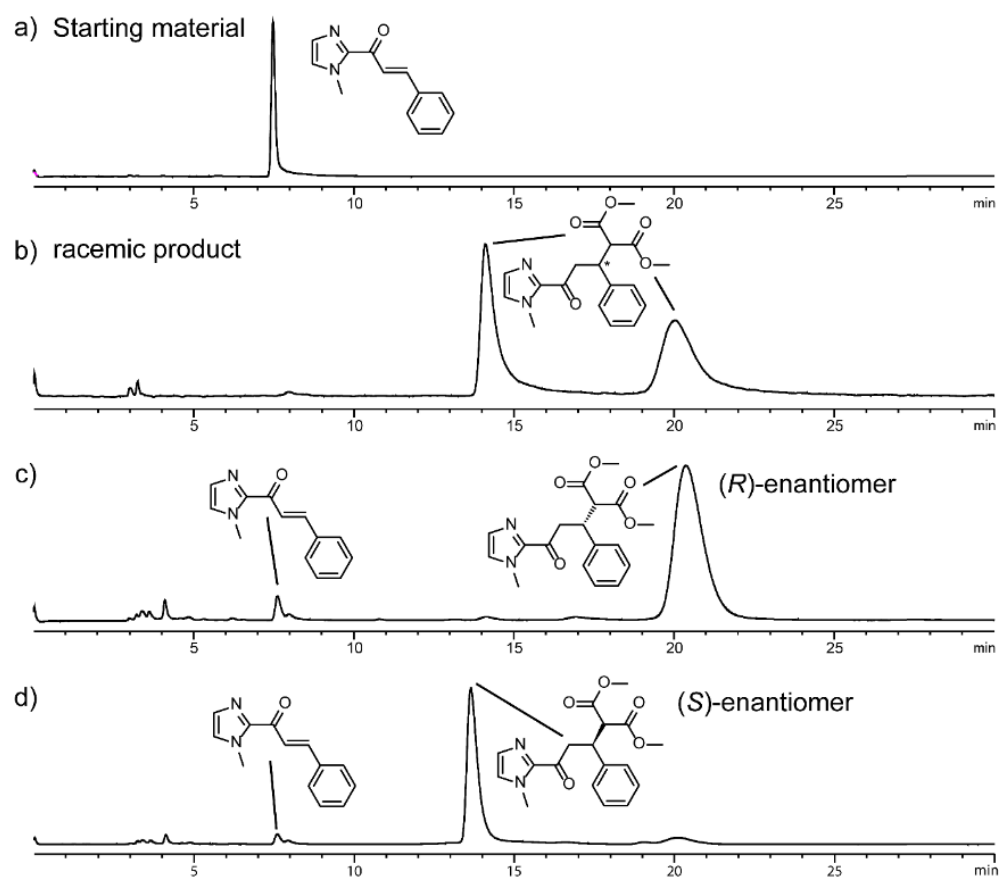

Figure S17. Analytic HPLC traces of a) MA1, b) racemic A2, reaction products of c) $120 \mu \mathrm{M}$ htelL ${ }_{3} \mathrm{D}_{1}, 100 \mu \mathrm{M} \mathrm{CuSO} 4,10 \mathrm{mM}$ HEPES pH 8, $100 \mathrm{mM} \mathrm{KCl,} 100 \mathrm{mM}$ DMM, $1 \mathrm{mM}$ substrate and $1 \% \mathrm{v} / \mathrm{v}$ DMSO, $5^{\circ} \mathrm{C}$, d) $120 \mu \mathrm{M}_{\text {htelL }}{ }_{2} \mathrm{G}, 100 \mu \mathrm{M} \mathrm{CuSO} 4,10$ $\mathrm{mM}$ HEPES $\mathrm{pH}$ 8, $100 \mathrm{mM} \mathrm{NaCl}, 100 \mathrm{mM}$ DMM, $1 \mathrm{mM}$ substrate and $1 \% \mathrm{v} / \mathrm{v}$ DMSO, $5^{\circ} \mathrm{C}$.
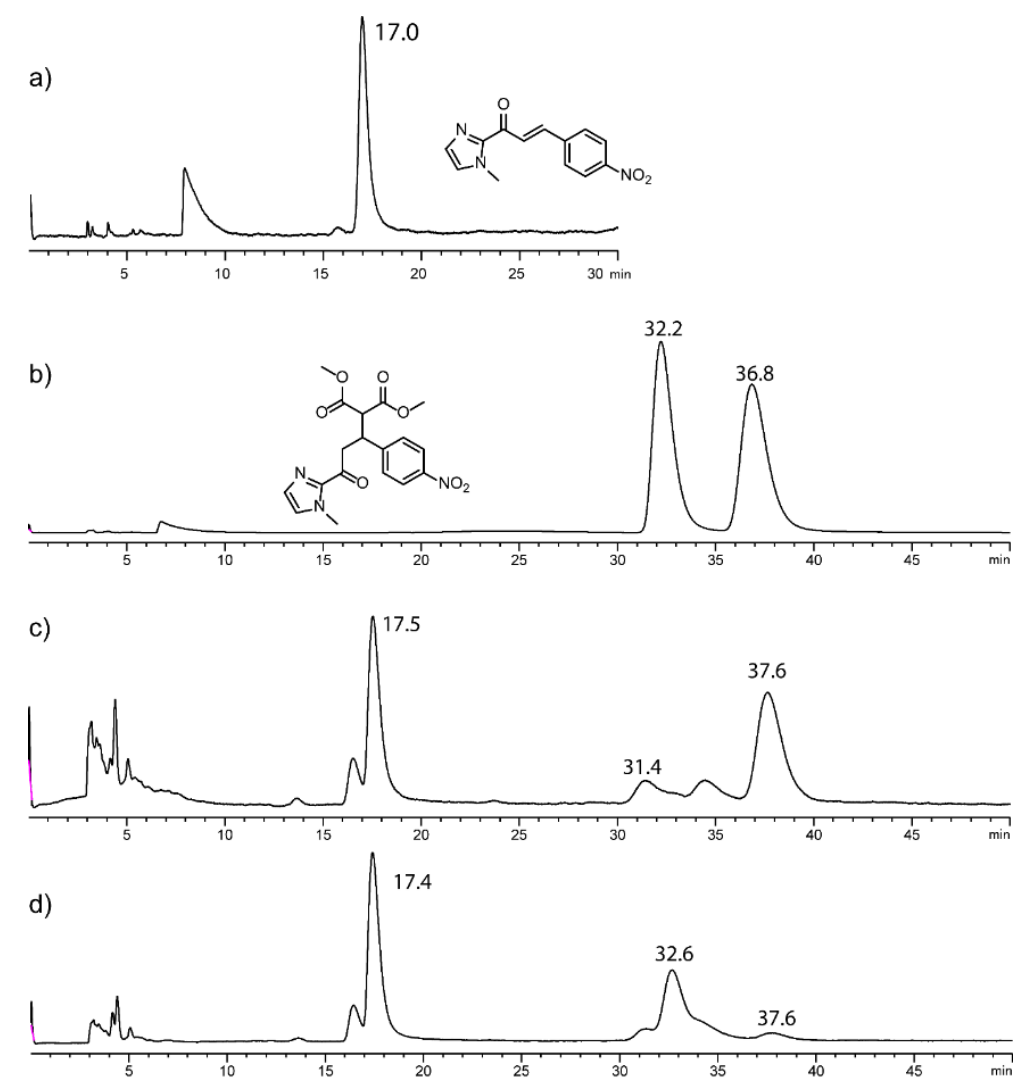

Figure S18. Analytic HPLC traces of a) MA2, b) racemic A2, reaction products of c) $120 \mu \mathrm{M}$ htelL ${ }_{3} \mathrm{D}, 100 \mu \mathrm{M} \mathrm{CuSO}, 10 \mathrm{mM}^{2}$ HEPES pH 8, $100 \mathrm{mM} \mathrm{KCl,} 100 \mathrm{mM}$ DMM, $1 \mathrm{mM}$ substrate and $4 \% \mathrm{v} / \mathrm{v}$ DMSO, $5^{\circ} \mathrm{C}$, d) $120 \mu \mathrm{M} \mathrm{htelL}^{R} 2 \mathrm{G}, 100 \mu \mathrm{M} \mathrm{CuSO} 4,10$ $\mathrm{mM}$ HEPES pH 8, $100 \mathrm{mM} \mathrm{NaCl}, 100 \mathrm{mM}$ DMM, $1 \mathrm{mM}$ substrate and $4 \% \mathrm{v} / \mathrm{v}$ DMSO, $5^{\circ} \mathrm{C}$. 

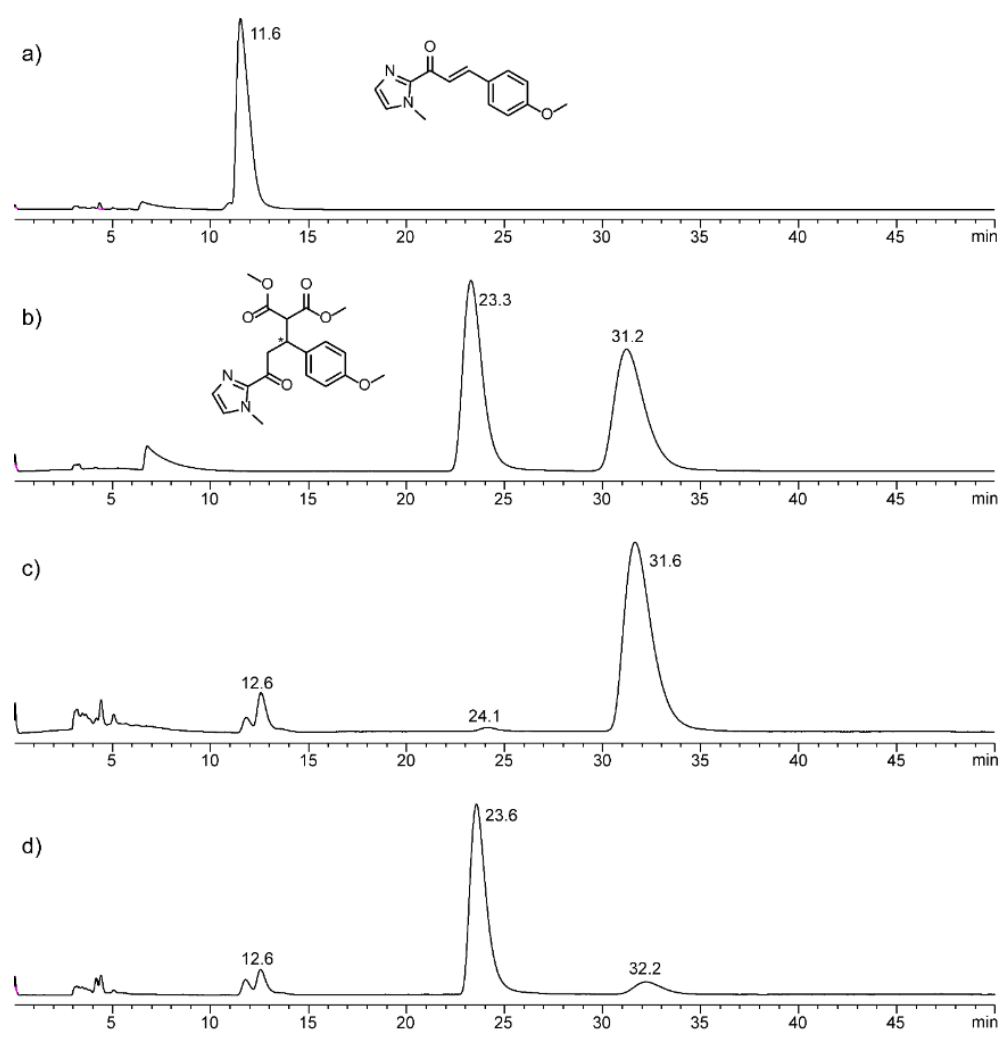

Figure S19. Analytic HPLC traces of a) MA3, b) racemic A3, reaction products of c) $120 \mu \mathrm{M}$ htelL $\mathbf{L}_{3} \mathrm{D}, 100 \mu \mathrm{M} \mathrm{CuSO}, 10 \mathrm{mM}$ HEPES pH 8, $100 \mathrm{mM} \mathrm{KCl}, 100 \mathrm{mM}$ DMM, $1 \mathrm{mM}$ substrate and $1 \% \mathrm{v} / \mathrm{v}$ DMSO, $5^{\circ} \mathrm{C}$, d) $120 \mu \mathrm{M} \mathrm{htelL}_{2}{ }_{2} \mathrm{G}, 100 \mu \mathrm{M} \mathrm{CuSO}_{4}, 10$ mM HEPES $\mathrm{pH}$ 8, $100 \mathrm{mM} \mathrm{NaCl}, 100 \mathrm{mM}$ DMM, $1 \mathrm{mM}$ substrate and $1 \% \mathrm{v} / \mathrm{v}$ DMSO, $5^{\circ} \mathrm{C}$.
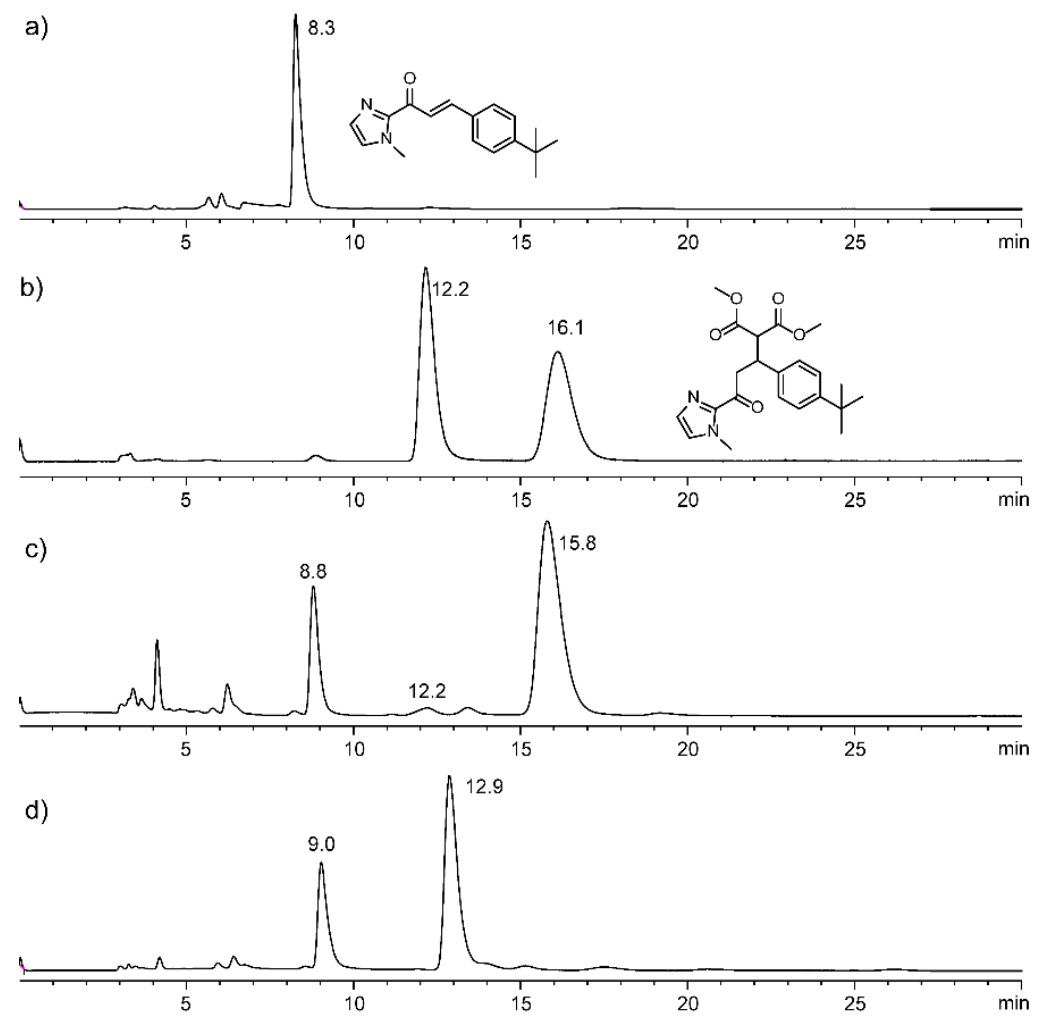

Figure S20. Analytic HPLC traces of a) MA4, b) racemic A4, reaction products of c) $120 \mu \mathrm{M}$ htelL ${ }_{3}{ }_{3} \mathrm{D}, 100 \mu \mathrm{M} \mathrm{CuSO}, 10 \mathrm{mM}^{2}$

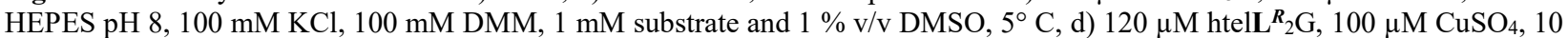
$\mathrm{mM}$ HEPES pH 8, $100 \mathrm{mM} \mathrm{NaCl}, 100 \mathrm{mM}$ DMM, $1 \mathrm{mM}$ substrate and $1 \% \mathrm{v} / \mathrm{v}$ DMSO, $5^{\circ} \mathrm{C}$. 

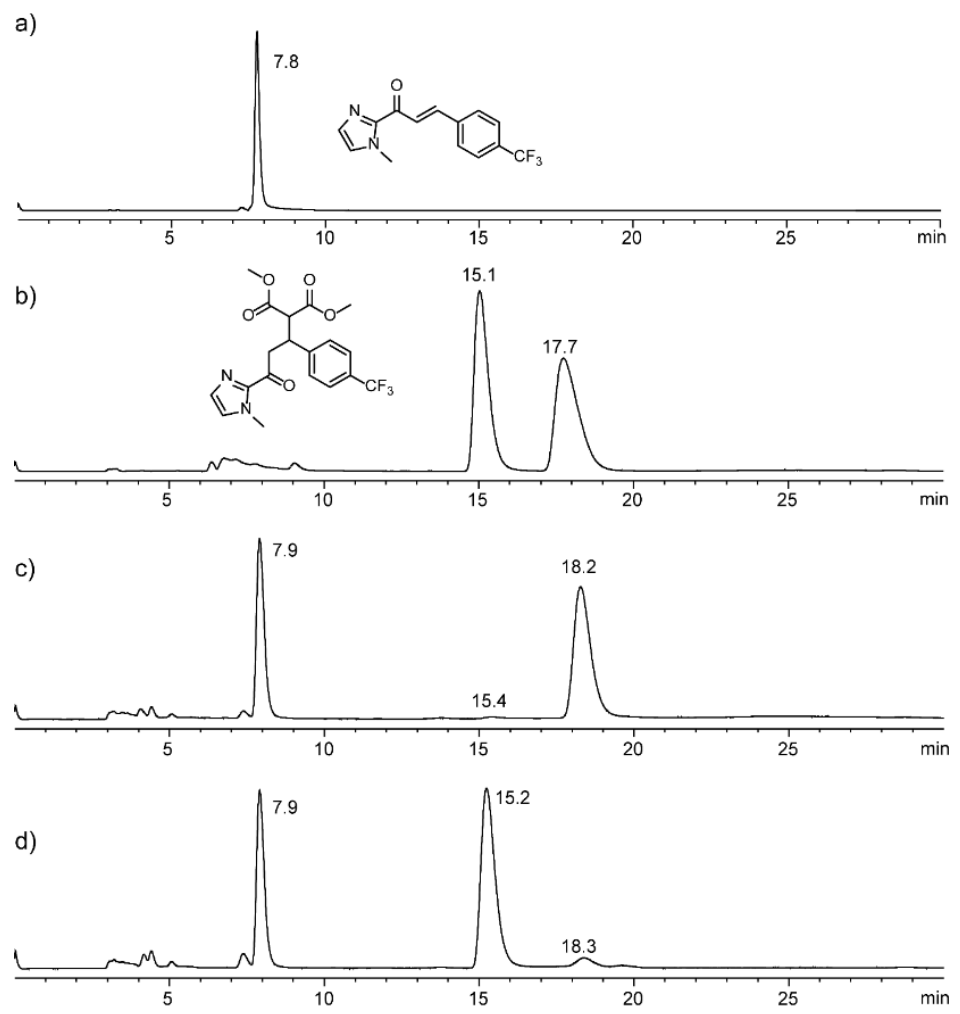

Figure S21. Analytic HPLC traces of a) MA5, b) racemic A5, reaction products of c) $120 \mu \mathrm{M}$ htelL ${ }_{3} \mathrm{D}, 100 \mu \mathrm{M} \mathrm{CuSO}, 10 \mathrm{mM}_{4}$

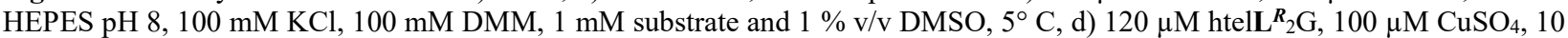
$\mathrm{mM}$ HEPES $\mathrm{pH} 8,100 \mathrm{mM} \mathrm{NaCl}, 100 \mathrm{mM}$ DMM, $1 \mathrm{mM}$ substrate and $1 \% \mathrm{v} / \mathrm{v}$ DMSO, $5^{\circ} \mathrm{C}$.

a)
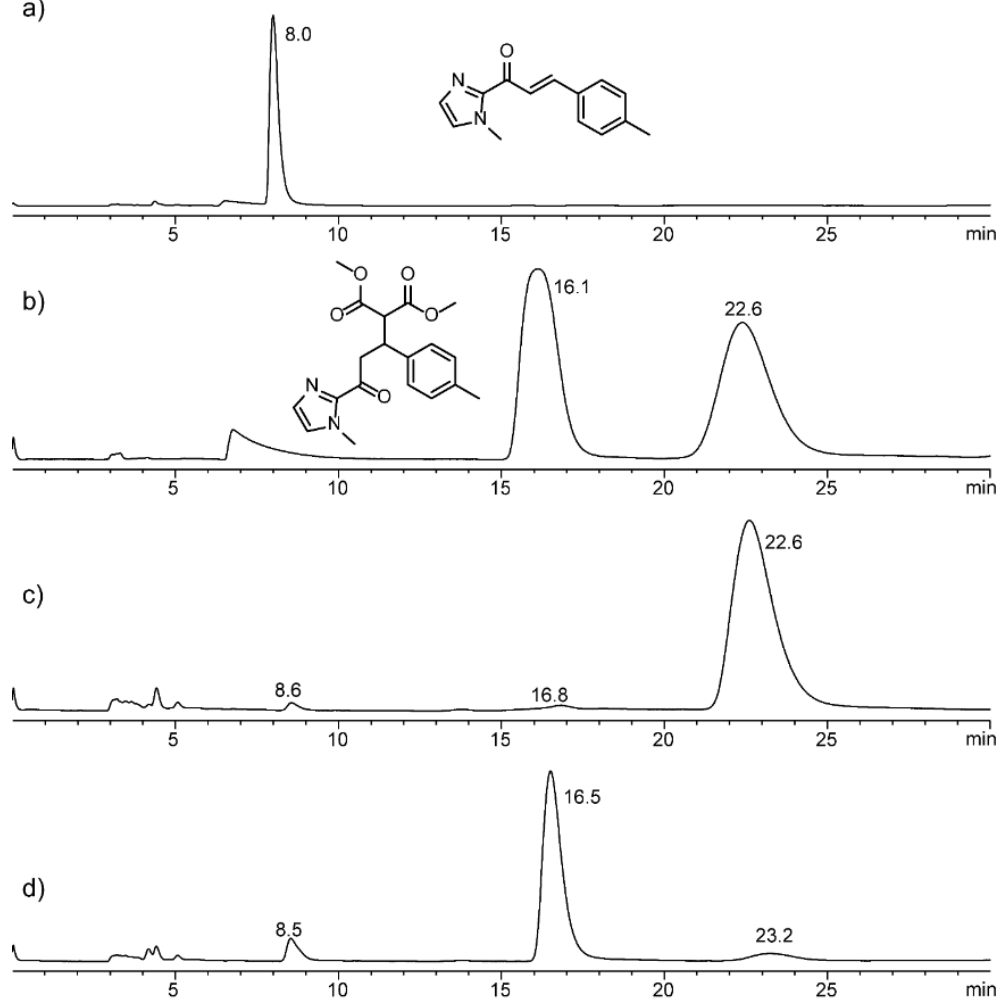

Figure S22. Analytic HPLC traces of a) MA6, b) racemic A6, reaction products of c) $120 \mu \mathrm{M}$ htelL ${ }_{3} \mathrm{D}_{1}, 100 \mu \mathrm{M} \mathrm{CuSO}, 10 \mathrm{mM}^{2}$ HEPES pH 8, $100 \mathrm{mM} \mathrm{KCl,} 100 \mathrm{mM}$ DMM, $1 \mathrm{mM}$ substrate and $1 \% \mathrm{v} / \mathrm{v}$ DMSO, $5^{\circ} \mathrm{C}$, d) $120 \mu \mathrm{M}$ htelL ${ }_{2}{ }_{2} \mathrm{G}, 100 \mu \mathrm{M} \mathrm{CuSO}_{4}, 10$ $\mathrm{mM}$ HEPES $\mathrm{pH}$ 8, $100 \mathrm{mM} \mathrm{NaCl}, 100 \mathrm{mM}$ DMM, $1 \mathrm{mM}$ substrate and $1 \% \mathrm{v} / \mathrm{v}$ DMSO, $5^{\circ} \mathrm{C}$. 

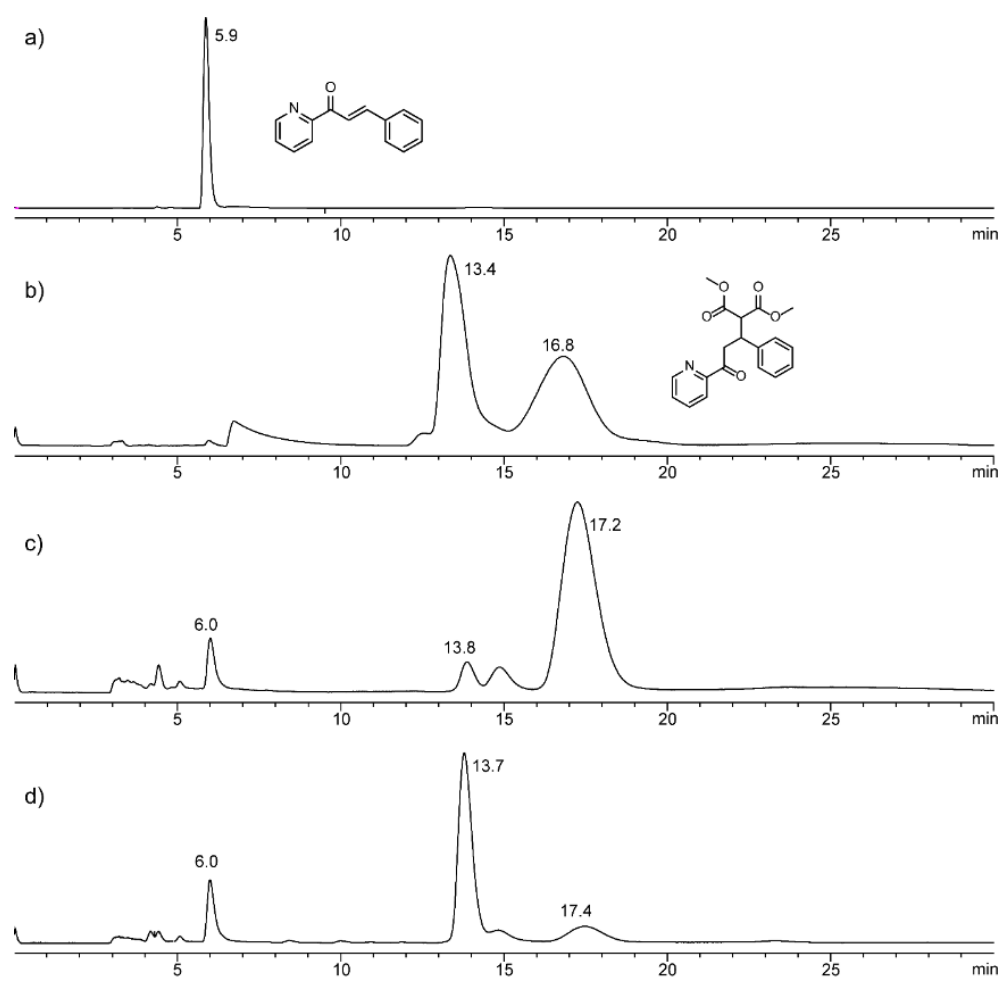

Figure S23. Analytic HPLC traces of a) MA7, b) racemic A7, reaction products of c) $120 \mu \mathrm{M}$ htelL ${ }_{3} \mathrm{D}, 100 \mu \mathrm{M} \mathrm{CuSO}, 10 \mathrm{mM}$

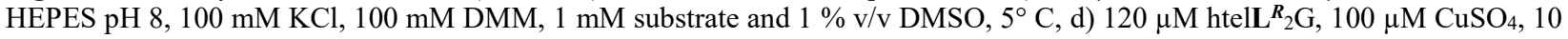
$\mathrm{mM}$ HEPES $\mathrm{pH} 8,100 \mathrm{mM} \mathrm{NaCl}, 100 \mathrm{mM}$ DMM, $1 \mathrm{mM}$ substrate and $1 \% \mathrm{v} / \mathrm{v}$ DMSO, $5^{\circ} \mathrm{C}$.

Table S3. Conversion and enantioselectivities of the investigated Michael-acceptors MA1 - 7. Conditions: $120 \mu \mathrm{M}$ DNA, $100 \mu \mathrm{M}$ CuSO4, $10 \mathrm{mM}$ HEPES pH 8, $100 \mathrm{mM}$ electrolyte, $100 \mathrm{mM}$ DMM, $1 \mathrm{mM}$ substrate and $1 \% \mathrm{v} / \mathrm{v}$ DMSO. $3 \mathrm{~d}$ incubation at $5^{\circ} \mathrm{C}$. The experiments were performed in duplicate. The reported error is the standard deviation from two experiments. $* 4 \% \mathrm{v} / \mathrm{v}$ DMSO.

\begin{tabular}{|c|c|c|c|c|}
\hline Seq. & electrolyte & Substrate & conversion & ee \\
\hline htelL ${ }^{R}{ }_{2} \mathrm{G}$ & $\mathrm{NaCl}$ & MA1 & $92 \pm 7$ & $90(S) \pm 1$ \\
\hline htel $\mathbf{L}^{R_{2}} \mathrm{G}$ & $\mathrm{NaCl}$ & MA2 & $17 \pm 1$ & $77(+) \pm 1$ \\
\hline htel $\mathbf{L}^{R_{2}} \mathrm{G}$ & $\mathrm{NaCl}$ & MA3 & $94 \pm 3$ & $81(+) \pm 1$ \\
\hline htel $\mathbf{L}^{R}{ }_{2} \mathrm{G}$ & $\mathrm{NaCl}$ & MA4 & $67 \pm 4$ & $95(+) \pm 1$ \\
\hline htel $\mathbf{L}^{R_{2} \mathrm{G}}$ & $\mathrm{NaCl}$ & MA5 & $73 \pm 5$ & $90(+) \pm 2$ \\
\hline htel $\mathbf{L}^{R_{2} \mathrm{G}}$ & $\mathrm{NaCl}$ & MA6 & $94 \pm 2$ & $89(+) \pm 2$ \\
\hline htel $\mathbf{L}^{\boldsymbol{R}}{ }_{2} \mathrm{G}$ & $\mathrm{NaCl}$ & MA7 & $98 \pm 1$ & $71(+) \pm 3$ \\
\hline htel $\mathbf{L}^{R_{3} \mathrm{D}}$ & $\mathrm{KCl}$ & MA1 & $94 \pm 7$ & $99(R) \pm 1$ \\
\hline htel $\mathbf{L}^{R_{3} \mathrm{D}}$ & $\mathrm{KCl}$ & MA2 & $34 \pm 2$ & $75(-) \pm 3$ \\
\hline htel $\mathbf{L}^{R_{3} \mathrm{D}}$ & $\mathrm{KCl}$ & MA3 & $90 \pm 8$ & $98(-) \pm 1$ \\
\hline htel $\mathbf{L}^{R_{3} \mathrm{D}}$ & $\mathrm{KCl}$ & MA4 & $74 \pm 8$ & $86(-) \pm 6$ \\
\hline htel $\mathbf{L}^{R_{3} \mathrm{D}}$ & $\mathrm{KCl}$ & MA5 & $59 \pm 9$ & $98(-) \pm 1$ \\
\hline htel $\mathbf{L}^{R}{ }_{3} \mathrm{D}$ & $\mathrm{KCl}$ & MA6 & $99 \pm 1$ & $99(-) \pm 1$ \\
\hline htel $\mathbf{L}^{R_{3} \mathrm{D}}$ & $\mathrm{KCl}$ & MA7 & $97 \pm 3$ & $90(-) \pm 1$ \\
\hline
\end{tabular}

Table S4. Conversion and enantioselectivity of htel $\mathbf{L}_{3}{ }_{3} \mathrm{~A}$-htelt $\mathbf{L}_{3} \mathrm{O}$. Conditions: $120 \mu \mathrm{M}$ DNA, $100 \mu \mathrm{M} \mathrm{CuSO}_{4}, 100$ $\mathrm{mM} \mathrm{KCl}, 10 \mathrm{mM}$ HEPES pH 8, $100 \mathrm{mM}$ DMM, $1 \mathrm{mM}$ Michael-acceptor 1 , DMSO $(1 \% \mathrm{v} / \mathrm{v})$ at $5{ }^{\circ} \mathrm{C}$ for $3 \mathrm{~d}$. 
Experiments were performed in duplicate if not otherwise mentioned. Positive ee values refer to the $(S)$ and negative values to the $(R)$ enantiomer. The reported error is the standard deviation from two experiments.

\begin{tabular}{|c|c|c|c|c|}
\hline Sequence & $L$ in Loop 1 & $L$ in Loop 3 & conversion [\%] & $e e[\%]$ \\
\hline htelL $S_{3} \mathrm{~A}$ & 2 & 1 & $28 \pm 6$ & (R) $31 \pm 1$ \\
\hline htell $\mathbf{L}_{3} \mathrm{E}$ & 2 & 1 & $16 \pm 1$ & (S) $27 \pm 3$ \\
\hline htell $S_{3}$ F & 2 & 1 & $8 \pm 1$ & (S) $11 \pm 1$ \\
\hline htel $\mathbf{L}_{3} \mathrm{D}$ & 1 & 2 & $80 \pm 7$ & (R) $91 \pm 1$ \\
\hline htell $\mathbf{L}_{3} \mathrm{C}$ & 1 & 2 & $40 \pm 2$ & (S) $57 \pm 2$ \\
\hline htell $\mathbf{L}_{3} \mathrm{G}$ & 1 & 2 & $66 \pm 4$ & (S) $44 \pm 2$ \\
\hline htel $\mathbf{L}_{3} \mathrm{~B}$ & 1 & 2 & $92 \pm 8$ & (R) $96 \pm 3$ \\
\hline htelL ${ }^{S_{3} \mathrm{H}}$ & 1 & 2 & $94 \pm 6$ & (R) $83 \pm 1$ \\
\hline htel. $\mathbf{L}_{3} \mathrm{I}$ & 1 & 2 & $87 \pm 2$ & (R) $91 \pm 3$ \\
\hline htell $\mathbf{L}_{3} \mathrm{~J}$ & 1 & 2 & $97 \pm 2$ & (R) $91 \pm 3$ \\
\hline htelL $S_{3 \mathrm{~K}}$ & 1 & 2 & $93 \pm 3$ & (R) $86 \pm 2$ \\
\hline $\operatorname{htel} \mathbf{L}_{3}{ }_{3}$ & 1 & 2 & $82 \pm 6$ & (R) $93 \pm 1$ \\
\hline htelL $S_{3} \mathrm{M}$ & 1 & 2 & $96 \pm 4$ & (R) $90 \pm 3$ \\
\hline htelL $\mathbf{L}_{3} \mathrm{~N}$ & 2 & 1 & $47 \pm 2$ & (R) $32 \pm 1$ \\
\hline htel $\mathbf{L}_{3} \mathrm{O}$ & 2 & 1 & $9 \pm 2$ & (R) $37 \pm 3$ \\
\hline
\end{tabular}

Table S5. Conversion and enantioselectivity of htel $\mathbf{L}_{3}{ }_{3} \mathrm{~A}-\mathrm{htel}_{\mathbf{L}} \boldsymbol{S}_{3} \mathrm{O}$. Conditions: $120 \mu \mathrm{M}$ DNA, $100 \mu \mathrm{M} \mathrm{CuSO}_{4}, 100$ $\mathrm{mM} \mathrm{NaCl}, 10 \mathrm{mM}$ HEPES pH 8, $100 \mathrm{mM}$ DMM, $1 \mathrm{mM}$ Michael-acceptor 1 , DMSO $(1 \% \mathrm{v} / \mathrm{v})$ at $5{ }^{\circ} \mathrm{C}$ for $3 \mathrm{~d}$. Experiments were performed in duplicate if not otherwise mentioned. Positive ee values refer to the $(S)$ and negative values to the $(R)$ enantiomer. The reported error is the standard deviation from two experiments.

\begin{tabular}{|c|c|c|c|c|}
\hline Sequence & $L$ in Loop 1 & $L$ in Loop 3 & conversion [\%] & $e e[\%]$ \\
\hline htelL $S_{3}$ A & 2 & 1 & $12 \pm 2$ & (S) $3 \pm 2$ \\
\hline htel $\mathbf{L}_{3} \mathrm{E}$ & 2 & 1 & $30 \pm 9$ & (S) $67 \pm 7$ \\
\hline htel $\mathbf{L}_{3} \mathrm{~F}$ & 2 & 1 & $3 \pm 2$ & (S) $14 \pm 2$ \\
\hline htel $L^{S_{3} \mathrm{D}}$ & 1 & 2 & $20 \pm 1$ & (R) $68 \pm 1$ \\
\hline htel $\mathbf{L}_{3} \mathrm{C}$ & 1 & 2 & $15 \pm 4$ & (S) $32 \pm 1$ \\
\hline htel $\mathbf{L}_{3} \mathrm{G}$ & 1 & 2 & $11 \pm \pm 1$ & (S) $48 \pm 3$ \\
\hline htel $\mathbf{L}_{3} \mathrm{~B}$ & 1 & 2 & $45 \pm 2$ & (R) $55 \pm 1$ \\
\hline htel $\mathbf{L}_{3} \mathrm{H}$ & 1 & 2 & $8 \pm 1$ & $(S) 3 \pm 1$ \\
\hline htelL $S_{3} \mathrm{I}$ & 1 & 2 & $22 \pm 1$ & (R) $53 \pm 3$ \\
\hline htelL $S_{3} \mathrm{~J}$ & 1 & 2 & $22 \pm 7$ & (R) $52 \pm 4$ \\
\hline htel $L_{3} S_{3}$ & 1 & 2 & $10 \pm 4$ & (R) $18 \pm 7$ \\
\hline htel $\mathbf{L}_{3} \mathrm{~L}$ & 1 & 2 & $65 \pm 4$ & (R) $45 \pm 1$ \\
\hline htelL ${ }^{S_{3} \mathrm{M}}$ & 1 & 2 & $22 \pm 4$ & (R) $55 \pm 10$ \\
\hline htel $L_{3} S_{3}$ & 2 & 1 & $12 \pm 1$ & (R) $37 \pm 1$ \\
\hline htelL $\mathbf{L}_{3} \mathrm{O}$ & 2 & 1 & $8 \pm 1$ & (R) $35 \pm 4$ \\
\hline
\end{tabular}

Table S6. Conversion and enantioselectivity of htelL $\mathbf{S}_{2} \mathrm{~A}$-htelt $\mathbf{L}_{2}{ }_{2} \mathrm{M}$. Conditions: $120 \mu \mathrm{M}$ DNA, $100 \mu \mathrm{M} \mathrm{CuSO}{ }_{4}, 100$ $\mathrm{mM} \mathrm{NaCl}, 10 \mathrm{mM}$ HEPES pH 8, $100 \mathrm{mM}$ DMM, $1 \mathrm{mM}$ Michael-acceptor 1 , DMSO (1\% v/v) at $5{ }^{\circ} \mathrm{C}$ for $3 \mathrm{~d}$. 
Experiments were performed in duplicate if not otherwise mentioned. Positive ee values refer to the $(S)$ and negative values to the $(R)$ enantiomer. The reported error is the standard deviation from two experiments.

\begin{tabular}{|c|c|c|c|c|}
\hline Sequence & L in Loop 1 & $L$ in Loop 3 & conversion [\%] & $e e[\%]$ \\
\hline htelL $S_{2} \mathrm{~B}$ & 1 & 1 & $26 \pm 2$ & (R) $9 \pm 8$ \\
\hline htel $\mathbf{L}_{2} \mathrm{C}$ & 1 & 1 & $56 \pm 3$ & (R) $7 \pm 1$ \\
\hline htelL $S_{2} \mathrm{D}$ & 1 & 1 & $16 \pm 1$ & (S) $4 \pm 3$ \\
\hline htell $\mathbf{L}_{2} \mathrm{~A}$ & 1 & 1 & $21 \pm 1$ & (S) $33 \pm 2$ \\
\hline htell $\mathbf{L}_{2} \mathrm{E}$ & 1 & 1 & $16 \pm 2$ & (S) $14 \pm 2$ \\
\hline htel. ${ }^{S_{2} \mathrm{~K}}$ & 1 & 1 & $16 \pm 1$ & (R) $11 \pm 4$ \\
\hline htel $\mathbf{L}_{2}{ }_{2} \mathrm{~F}$ & 2 & 0 & $78 \pm 7$ & (S) $71 \pm 2$ \\
\hline htell $\mathbf{L}_{2} \mathrm{G}$ & 2 & 0 & $61 \pm 2$ & (S) $67 \pm 1$ \\
\hline htell $\mathbf{L}_{2} \mathrm{H}$ & 2 & 0 & $67 \pm 2$ & (S) $61 \pm 1$ \\
\hline htell $\mathbf{L}_{2} \mathrm{I}$ & 0 & 2 & $43 \pm 1$ & (S) $44 \pm 2$ \\
\hline $\operatorname{htel} S_{2} \mathrm{~J}$ & 0 & 2 & $40 \pm 1$ & (S) $20 \pm 1$ \\
\hline htel $\mathbf{L}_{2} S_{\mathrm{L}}$ & 1 & 1 & $14 \pm 1$ & (R) $46 \pm 1$ \\
\hline htelL ${ }^{S_{2} \mathrm{M}}$ & 1 & 1 & $6 \pm 1$ & (R) $21 \pm 4$ \\
\hline
\end{tabular}

Table S7. Conversion and enantioselectivity of htel $\mathbf{L}_{2}{ }_{2} \mathrm{~A}-\mathrm{hte} \mathrm{LL}_{2}{ }_{2} \mathrm{M}$. Conditions: $120 \mu \mathrm{M}$ DNA, $100 \mu \mathrm{M} \mathrm{CuSO}_{4}, 100$ $\mathrm{mM} \mathrm{KCl}, 10 \mathrm{mM}$ HEPES pH 8, $100 \mathrm{mM}$ DMM, $1 \mathrm{mM}$ Michael-acceptor 1 , DMSO $(1 \% \mathrm{v} / \mathrm{v})$ at $5{ }^{\circ} \mathrm{C}$ for $3 \mathrm{~d}$. Experiments were performed in duplicate if not otherwise mentioned. Positive ee values refer to the $(S)$ and negative values to the $(R)$ enantiomer. The reported error is the standard deviation from two experiments.

\begin{tabular}{|c|c|c|c|c|}
\hline Sequence & L in Loop 1 & $\mathrm{~L}$ in Loop 3 & conversion [\%] & $e e[\%]$ \\
\hline htelL ${ }^{S_{2} \mathrm{~B}}$ & 1 & 1 & $35 \pm 6$ & (S) $13 \pm 4$ \\
\hline htel: $\mathbf{L}_{2} \mathrm{C}$ & 1 & 1 & $61 \pm 4$ & (R) $28 \pm 1$ \\
\hline htel $\mathbf{L}_{2}{ }_{2} \mathrm{D}$ & 1 & 1 & $52 \pm 6$ & (R) $16 \pm 7$ \\
\hline htel: $\mathbf{L}_{2} \mathrm{~A}$ & 1 & 1 & $28 \pm 2$ & (S) $31 \pm 1$ \\
\hline htell $L_{2} \mathrm{E}$ & 1 & 1 & $33 \pm 4$ & (S) $28 \pm 1$ \\
\hline htell $\mathrm{L}_{2} \mathrm{~K}$ & 1 & 1 & $68 \pm 2$ & (R) $40 \pm 1$ \\
\hline htell $S_{2} \mathrm{~F}$ & 2 & 0 & $75 \pm 2$ & (S) $46 \pm 1$ \\
\hline htell $\mathbf{L}_{2} \mathrm{G}$ & 2 & 0 & $69 \pm 4$ & (R) $29 \pm 1$ \\
\hline htell $\mathbf{S}_{2} \mathrm{H}$ & 2 & 0 & $73 \pm 1$ & (S) $4 \pm 1$ \\
\hline htel: $\mathbf{L}_{2} \mathrm{I}$ & 0 & 2 & $97 \pm 2$ & (R) $71 \pm 2$ \\
\hline htell $S_{2} \mathbf{J}$ & 0 & 2 & $92 \pm 2$ & (S) $44 \pm 2$ \\
\hline htelL $S_{2} \mathrm{~L}$ & 1 & 1 & $53 \pm 7$ & $(S) 6 \pm 2$ \\
\hline htelL $S_{2} \mathrm{M}$ & 1 & 1 & $18 \pm 3$ & (R) $15 \pm 3$ \\
\hline
\end{tabular}


Table S8. Conversion and enantioselectivity of selected G-quadruplexes containing the $\mathbf{L}^{\boldsymbol{R}}$ enantiomer. Conditions: 120 $\mu \mathrm{M}$ DNA, $100 \mu \mathrm{M} \mathrm{CuSO}_{4}, 100 \mathrm{mM}$ electrolyte, $10 \mathrm{mM}$ HEPES pH 8, $100 \mathrm{mM}$ DMM, $1 \mathrm{mM}$ Michael-acceptor 1, DMSO $(1 \% \mathrm{v} / \mathrm{v})$ at $5{ }^{\circ} \mathrm{C}$ for $3 \mathrm{~d}$. Experiments were performed in duplicate if not otherwise mentioned. Positive ee values refer to the $(S)$ and negative values to the $(R)$ enantiomer. The reported error is the standard deviation from two experiments.

\begin{tabular}{|c|c|c|c|c|}
\hline Sequence & Number $\mathbf{L}$ & electrolyte & conversion & $e e[\%]$ \\
\hline htelL $\mathbf{L}^{R_{2}} \mathrm{G}$ & 2 & $\mathrm{KCl}$ & $90 \pm 2$ & (S) $54 \pm 3$ \\
\hline htel. $\mathbf{L}^{R} \mathrm{I}$ & 2 & $\mathrm{KCl}$ & $92 \pm 1$ & (R) $85 \pm 2$ \\
\hline htel. $\mathbf{L}^{R_{3} G}$ & 3 & $\mathrm{KCl}$ & $60 \pm 6$ & (S) $4 \pm 2$ \\
\hline htel $\mathbf{L}^{R_{3} \mathrm{~B}}$ & 3 & $\mathrm{KCl}$ & $97 \pm 1$ & (R) $84 \pm 1$ \\
\hline htel $\mathbf{L}^{R_{3} \mathrm{D}}$ & 3 & $\mathrm{KCl}$ & $94 \pm 7$ & $\geq(R) 99 \pm 1$ \\
\hline htel $\mathbf{L}^{R_{2}} \mathrm{~F}$ & 2 & $\mathrm{KCl}$ & $94 \pm 2$ & (S) $30 \pm 1$ \\
\hline htel $\mathbf{L}^{R}{ }_{2} \mathrm{H}$ & 2 & $\mathrm{KCl}$ & $93 \pm 1$ & (S) $40 \pm 1$ \\
\hline htel $\mathbf{L}^{R_{3} \mathrm{H}}$ & 3 & $\mathrm{KCl}$ & $95 \pm 1$ & (R) $68 \pm 1$ \\
\hline htel $\mathbf{L}^{R_{3} \mathrm{I}}$ & 3 & $\mathrm{KCl}$ & $98 \pm 1$ & (R) $83 \pm 1$ \\
\hline htel $\mathbf{L}^{R_{3} \mathrm{~J}}$ & 3 & $\mathrm{KCl}$ & $96 \pm 4$ & (R) $85 \pm 1$ \\
\hline htel $\mathbf{L}^{R}{ }_{3} \mathrm{~K}$ & 3 & $\mathrm{KCl}$ & $94 \pm 2$ & $(R) 67 \pm 1$ \\
\hline htel. $\mathbf{L}^{\boldsymbol{R}}{ }_{3} \mathrm{~L}$ & 3 & $\mathrm{KCl}$ & $96 \pm 1$ & (R) $83 \pm 1$ \\
\hline htel. $\mathbf{L}^{R}{ }_{3} \mathrm{M}$ & 3 & $\mathrm{KCl}$ & $98 \pm 2$ & (R) $83 \pm 1$ \\
\hline htel. $\mathbf{L}^{\boldsymbol{R}}{ }_{2} \mathrm{G}$ & 2 & $\mathrm{NaCl}$ & $92 \pm 7$ & (S) $90 \pm 1$ \\
\hline htel $\mathbf{L}^{R}{ }_{2} \mathrm{I}$ & 2 & $\mathrm{NaCl}$ & $95 \pm 3$ & (R) $7 \pm 1$ \\
\hline htel $\mathbf{L}^{R_{2}} \mathrm{~F}$ & 2 & $\mathrm{NaCl}$ & $95 \pm 4$ & (S) $86 \pm 1$ \\
\hline htel $\mathbf{L}^{R}{ }_{2} \mathrm{H}$ & 2 & $\mathrm{NaCl}$ & $93 \pm 1$ & (S) $56 \pm 1$ \\
\hline
\end{tabular}

Table S9. Conversion and enantioselectivity of selected G-quadruplexes containing no $\mathbf{L}$ or $\geq$ four times $\mathbf{L}$. Conditions: $120 \mu \mathrm{M}$ DNA, $100 \mu \mathrm{M} \mathrm{CuSO}_{4}, 100 \mathrm{mM}$ electrolyte, $10 \mathrm{mM}$ HEPES pH 8, $100 \mathrm{mM}$ DMM, $1 \mathrm{mM}$ Michael-acceptor 1 , DMSO $(1 \% \mathrm{v} / \mathrm{v})$ at $5{ }^{\circ} \mathrm{C}$ for $3 \mathrm{~d}$. Experiments were performed in duplicate if not otherwise mentioned. *Experiment was performed once. Positive ee values refer to the $(S)$ and negative values to the $(R)$ enantiomer. The reported error is the standard deviation from two experiments.

\begin{tabular}{|c|c|c|c|c|}
\hline Sequence & Number L & electrolyte & conversion & $e e[\%]$ \\
\hline- & - & $\mathrm{NaCl}$ & $15 \pm 5$ & 0 \\
\hline- & - & $\mathrm{KCl}$ & $23 \pm 1$ & 0 \\
\hline c-Myc & 0 & $\mathrm{KCl}$ & $12 \pm 2$ & (R) $1 \pm 1$ \\
\hline htel $_{22}$ & 0 & $\mathrm{KCl}$ & $10 \pm 1$ & (S) $5 \pm 7$ \\
\hline c-Myc* & 0 & $\mathrm{NaCl}$ & 5 & 6 \\
\hline $\operatorname{htel}_{22} *$ & 0 & $\mathrm{NaCl}$ & 7 & 0 \\
\hline htelL ${ }^{S_{4} \mathrm{~A}}$ & 4 & $\mathrm{KCl}$ & $10 \pm 1$ & (R) $25 \pm 2$ \\
\hline htel $\mathbf{L}_{4}{ }_{4} \mathrm{~B}$ & 4 & $\mathrm{KCl}$ & $4 \pm 2$ & (S) $14 \pm 6$ \\
\hline htel $\mathbf{L}^{S_{4} \mathrm{C}}$ & 4 & $\mathrm{KCl}$ & $7 \pm 5$ & (R) $28 \pm 2$ \\
\hline htel $\mathbf{L}_{4}{ }_{4} \mathrm{~B}$ & 4 & $\mathrm{NaCl}$ & $2 \pm 1$ & (S) $53 \pm 8$ \\
\hline htel. $\mathbf{L}_{4} \mathrm{C}$ & 4 & $\mathrm{NaCl}$ & 0 & 0 \\
\hline $\operatorname{htel}^{S_{6}}$ & 6 & $\mathrm{KCl}$ & 0 & 0 \\
\hline htelL ${ }^{S_{7}}$ & 7 & $\mathrm{KCl}$ & $17 \pm 3$ & (R) $27 \pm 3$ \\
\hline
\end{tabular}


Table S10. Conversion and enantioselectivity of htel $\mathbf{L}^{\boldsymbol{R}_{3} \mathrm{D}}$ and htel $\mathbf{L}^{\boldsymbol{R}}{ }_{2} \mathrm{G}$ in absence of transition metals and in presence of $\mathrm{ZnI}$. Conditions: $120 \mu \mathrm{M}$ DNA, $100 \mu \mathrm{M}$ ZnI, $100 \mathrm{mM}$ electrolyte, $10 \mathrm{mM}$ HEPES pH 8, $100 \mathrm{mM}$ DMM, 1 mM Michael-acceptor 1, $\operatorname{DMSO}(1 \% \mathrm{v} / \mathrm{v})$ at $5{ }^{\circ} \mathrm{C}$ for $3 \mathrm{~d}$. Experiments were performed in duplicate if not otherwise mentioned. *Experiment was performed once. The reported error is the standard deviation from two experiments. $\mathbf{L}^{S}$ corresponds to the free GNA nucleoside of $\mathbf{L}^{R}$. The stereo configuration is formally inverted due to a change of the priorities at the stereo centre for the free nucleoside.

\begin{tabular}{|c|c|c|c|c|c|}
\hline Sequence & Number $\mathbf{L}$ & electrolyte & transition metal salt & conversion [\%] & $e e[\%]$ \\
\hline $91 \mathbf{L}^{R}$ & 3 & $\mathrm{KCl}$ & $\mathrm{CuSO}_{4}$ & 0 & 0 \\
\hline $92 \mathbf{L}^{R}$ & 2 & $\mathrm{NaCl}$ & $\mathrm{CuSO}_{4}$ & 0 & 0 \\
\hline $\begin{array}{l}\text { N-methyl imidazole ( } 480 \\
\mu \mathrm{M})\end{array}$ & - & $\mathrm{KCl}$ & $\mathrm{CuSO}_{4}$ & $30( \pm 1)$ & 0 \\
\hline $\begin{array}{l}\text { N-methyl imidazole } \\
(480 \mu \mathrm{M})\end{array}$ & - & $\mathrm{NaCl}$ & $\mathrm{CuSO}_{4}$ & $29( \pm 5)$ & 0 \\
\hline $\mathbf{L}^{S}(120 \mu \mathrm{M})$ & - & $\mathrm{KCl}$ & $\mathrm{CuSO}_{4}$ & $22( \pm 2)$ & (S) $3 \pm 1$ \\
\hline $\mathbf{L}^{S}(240 \mu \mathrm{M})$ & - & $\mathrm{KCl}$ & $\mathrm{CuSO}_{4}$ & $24( \pm 1)$ & (S) $2 \pm 2$ \\
\hline $\mathrm{L}^{S}(360 \mu \mathrm{M})$ & - & $\mathrm{KCl}$ & $\mathrm{CuSO}_{4}$ & $25( \pm 4)$ & (S) $1 \pm 1$ \\
\hline $\mathbf{L}^{S}(480 \mu \mathrm{M})$ & - & $\mathrm{KCl}$ & $\mathrm{CuSO}_{4}$ & $24( \pm 3)$ & (S) $1 \pm 3$ \\
\hline htell ${ }^{R} \mathrm{D}^{*}$ & 3 & $\mathrm{KCl}$ & $\mathrm{ZnI} 2$ & - & - \\
\hline htel. $\mathbf{L}_{2} \mathrm{G}^{*}$ & 2 & $\mathrm{NaCl}$ & $\mathrm{ZnI} 2$ & - & - \\
\hline htel. $\mathbf{L}_{3} \mathrm{D}^{*}$ & 3 & $\mathrm{KCl}$ & - & - & - \\
\hline htell $\mathbf{L}_{2} \mathrm{G}^{*}$ & 2 & $\mathrm{NaCl}$ & - & - & - \\
\hline htell $\mathbf{L}_{3}{ }_{3} \mathrm{D}$ & 3 & $\mathrm{LiCl}$ & $\mathrm{CuSO}_{4}$ & 0 & 0 \\
\hline htel $\mathbf{L}^{R_{3} \mathrm{D}}$ & 3 & $\mathrm{NH}_{4} \mathrm{Cl}$ & $\mathrm{CuSO}_{4}$ & 0 & 0 \\
\hline htel $\mathbf{L}^{R_{2}} \mathrm{G}$ & 2 & $\mathrm{LiCl}$ & $\mathrm{CuSO}_{4}$ & $2( \pm 1)$ & n.d. \\
\hline htel $\mathbf{L}^{R_{2} \mathrm{G}}$ & 2 & $\mathrm{NH}_{4} \mathrm{Cl}$ & $\mathrm{CuSO}_{4}$ & $5( \pm 1)$ & n.d. \\
\hline
\end{tabular}

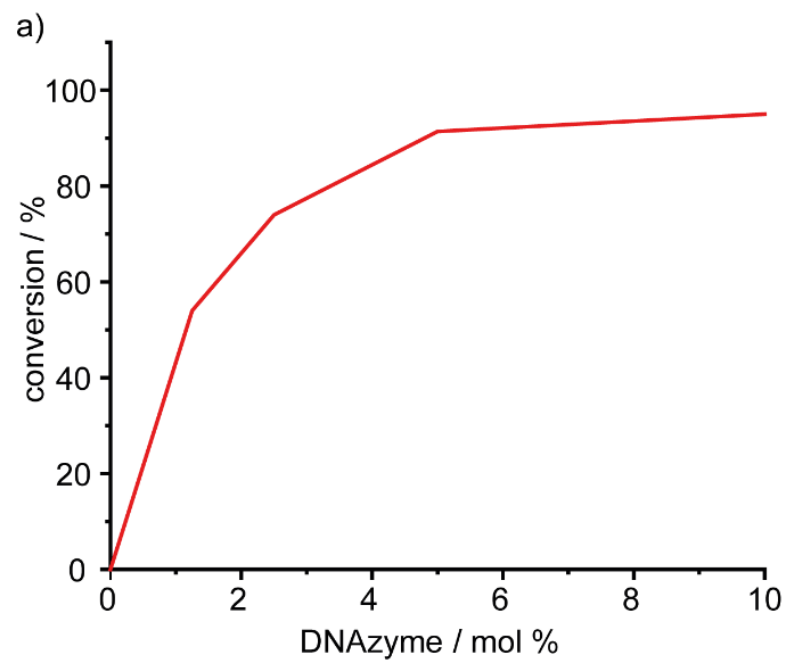

Figure S24. Conversion of the Michael-Addition after two days with varying concentrations of htelL $\boldsymbol{S}_{3} \mathrm{~B}$. Conditions: $100 \mathrm{mM} \mathrm{KCl}$, $10 \mathrm{mM}$ HEPES pH 8, 1 equiv. $\mathrm{CuSO}_{4}, 1 \mathrm{mM}$ Michael-acceptor, $1 \% \mathrm{v} / \mathrm{v}$ DMSO and $100 \mathrm{mM} \mathrm{DMM}$ at $5{ }^{\circ} \mathrm{C}$. 


\section{Kinetic studies by HPLC}

For kinetic studies at the same substrate concentrations (but lower DNA and $\mathrm{Cu}(\mathrm{II})$ concentrations) as for the tabulated conversion and ee determinations, individual samples at different timesteps were analysed (average of two experiments). Therefore, samples were prepared as described above and the Michael-addition was initiated by the addition of the Michael-acceptor and DMM. Conversion and enantioselectivity of the individual time steps were analysed as described above by chiral HPLC. The product formation was calculated, assuming that starting material is exclusively converted to the expected product. The onset of product formation was then plotted against the time and fitted via linear regression. From the slope of the linear fit $v$ was determined and $k_{c a t}$ by dividing $v$ with the concentration of the active DNAzyme (corresponding to the $\mathrm{Cu}^{\mathrm{II}}$ concentration).
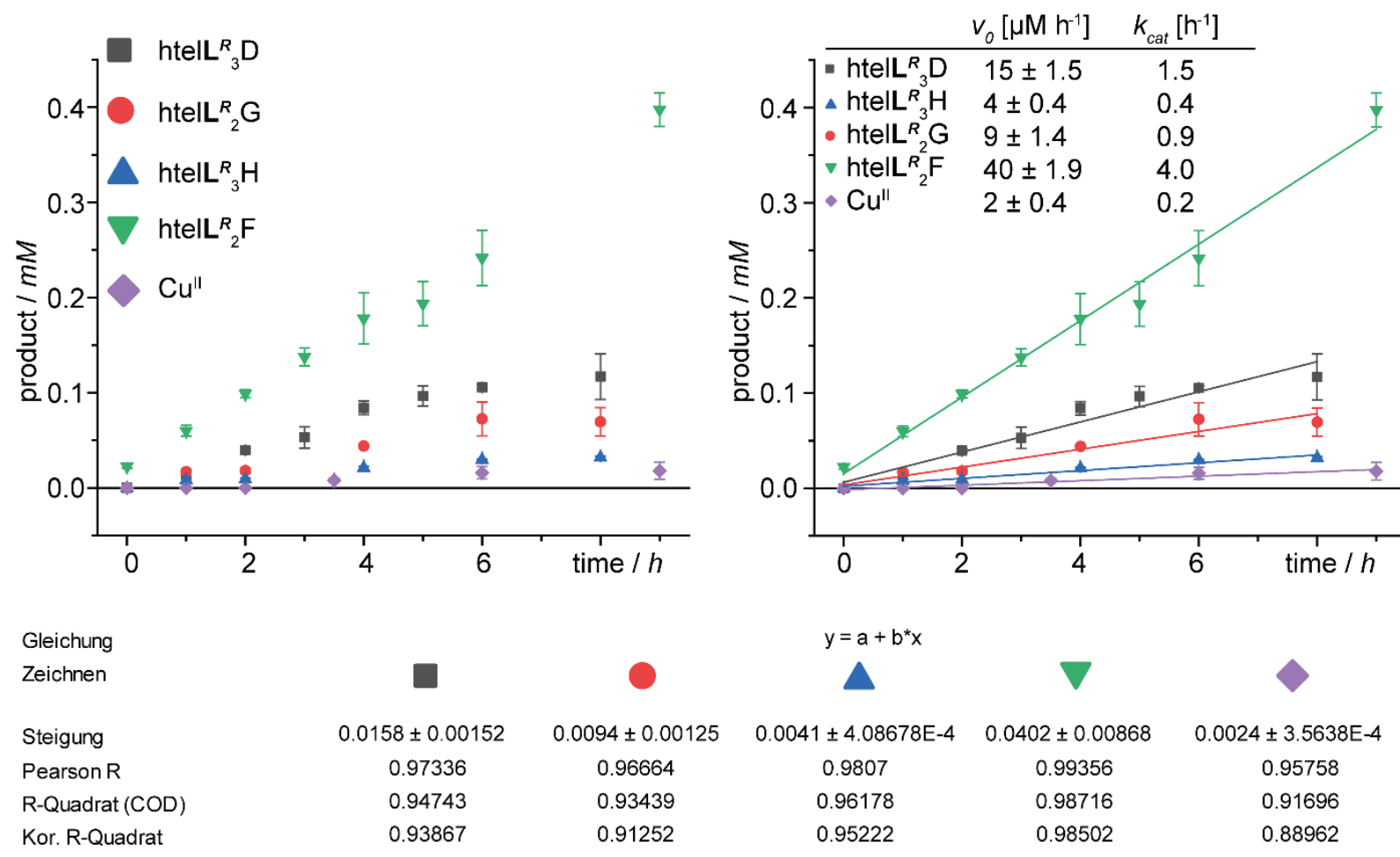

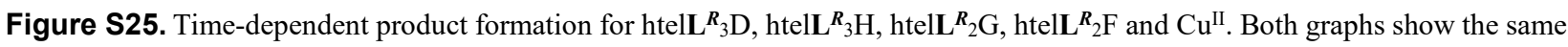
data, the right graph includes the linear fits. Every data point is the average of two independent experiments. $12 \mu \mathrm{M}$ G-quadruplex, $10 \mu \mathrm{M} \mathrm{CuSO}_{4}, 10 \mathrm{mM}$ HEPES pH 8, $100 \mathrm{mM} \mathrm{KCl}\left(\right.$ three $\mathbf{L}^{\boldsymbol{R}}$ ) or $\mathrm{NaCl}$ (two $\mathbf{L}^{\boldsymbol{R}}$ ), $100 \mathrm{mM} \mathrm{DMM}, 1 \mathrm{mM} \mathrm{MA1}$ and $1 \% \mathrm{v} / \mathrm{v} \mathrm{DMSO}$, $5^{\circ} \mathrm{C}$. 


\section{Kinetic studies to ascertain Michaelis-Menten saturation behaviour}

Kinetic studies at different, low starting concentrations of the substrate MA1 were carried out on Jasco V-650 and Jasco $V$-750 UV/Vis spectrometers using quartz glass cuvettes. At first, substrate MA1 and the DMM adduct A1 were characterized by UV/Vis spectroscopy at varying concentrations ( $2 \mathrm{~nm}$ bandwidth, $1 \mathrm{~nm}$ data interval $1 \mathrm{~cm}$ pathlength). From the difference spectrum, the maximum change was found at $336 \mathrm{~nm}$, a wavelength that is not interfering with the absorption of HEPES and DNA htel $\mathbf{L}^{R_{3}} \mathrm{D}$. The extinction coefficient $\varepsilon_{336}$ was determined from linear regression of the absorption at $336 \mathrm{~nm}$. For MA1, a significantly larger $\varepsilon_{336}=21600 \mathrm{M}^{-1} \mathrm{~cm}^{-1}$ was determined compared to $\varepsilon_{336}=$ $558 \mathrm{M}^{-1} \mathrm{~cm}^{-1}$ for $\mathbf{A 1}$. The increase of absorption was found to be linear between 0.0025 and $0.05 \mathrm{mM}$ and was chosen for kinetic studies. The substrate concentration range for this experiment was carefully optimized in a way, that both substrate and product are completely soluble over the entire length of the experiments in order to avoid deceiving plateau effects at higher substrate concentrations resulting from exceeding solubility limits. For the same reason, the DMSO content was raised to $10 \%$ and the temperature raised to $25^{\circ}$ (optimization of proper solubility was monitored by UV-Vis and NMR experiments). Hence, the obtained reaction rates cannot be directly compared to the values determined by the HPLC method described above.

To follow the reaction over time, the decrease of absorption at $\mathrm{A}_{336}$ was monitored, considering that only the Michaelacceptor MA1 is having a significant contribution to the absorption at $336 \mathrm{~nm}$ (Figure S26 and S27). The change at $336 \mathrm{~nm}$ was fitted by linear regression for different substrate starting concentrations [S] (one example shown in Figure $\mathrm{S} 28 \mathrm{c}$ ). From the slope, the reaction rate $v$ was calculated, using the extinction coefficient $\varepsilon_{336}=21600 \mathrm{M}^{-1} \mathrm{~cm}^{-1}$ of MA1. Rate $v$ was plotted against the substrate starting concentration and fitted using equation 1, describing a typical Michaelis-Menten kinetics model. Therefore, the corresponding preconfigured function of the software Origin 2019 was employed. From the fitting, a value of $K_{M}=35.2 \mu \mathrm{M}$ (albeit with a significant error) was determined for the $\mathrm{Cu}$ DNA-catalyzed reaction with MA1 (Figure S28a, black curve), while no Michaelis-Menten behaviour could be observed for the reaction catalyzed by free $\mathrm{Cu}$ (II) cations (Figure S28a, red curve: notably, the algorithm applied a linear fit and hence refused to determine $v_{\max }$ and $K_{M}$ values in this case; Figure S28b). Furthermore, assuming $k_{-1}>>$ $k_{\text {cat }}$ for a quickly exchanging substrate on the kinetically labile $\mathrm{Cu}(\mathrm{II})$ complex, we estimate $K_{d} \approx K_{M}$ as our attempts to determine $K_{d}$ directly by ITC and UV/Vis spectroscopy were hampered by unacceptable signal-to-noise ratios due to miniscule thermal effects and spectral changes, respectively, at the low concentration dictated by the substrate solubility.

\section{Equation 1}

$$
v=\frac{d[P]}{d t}=v_{\max } \frac{[S]}{K_{M}+[S]}
$$


a)

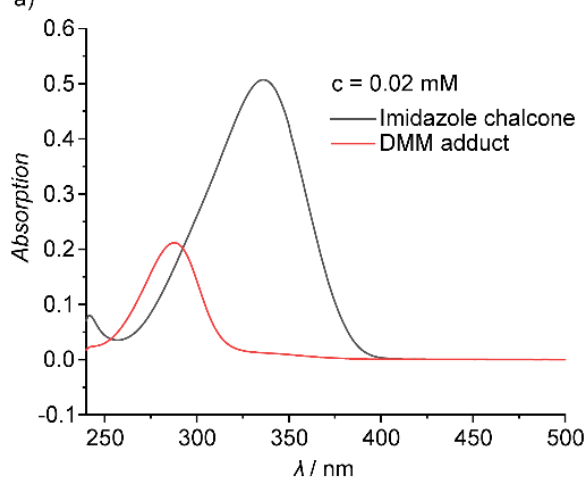

b)

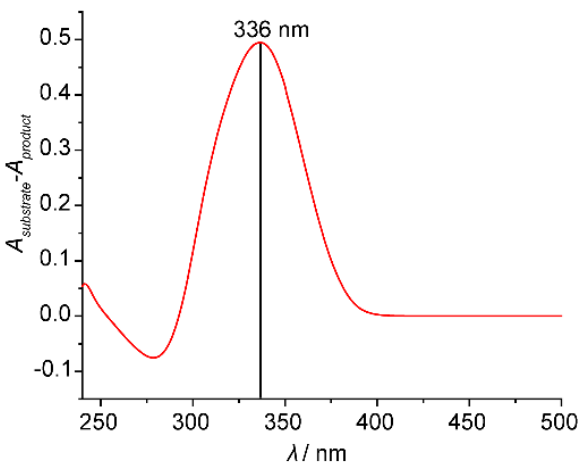

Figure S26. UV/Vis spectra a) of Michael-acceptor MA1 and the DMM adduct $A_{1}$ and b) the difference spectrum, where the absorption of the product (Al) was subtracted from the substrate (MAI) absorption. $\mathrm{H}_{2} \mathrm{O}$ :DMSO, 9:1, $20^{\circ} \mathrm{C}$.

a)

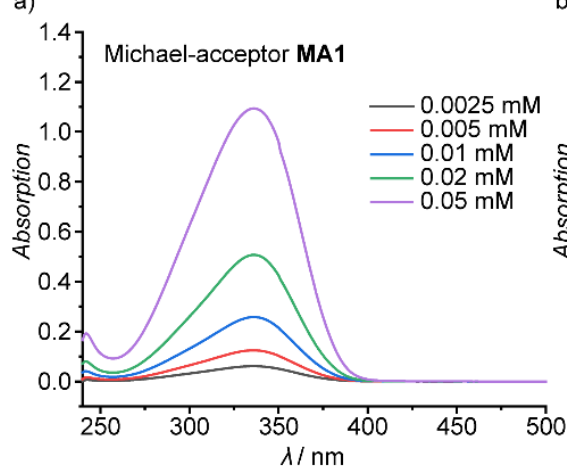

b)

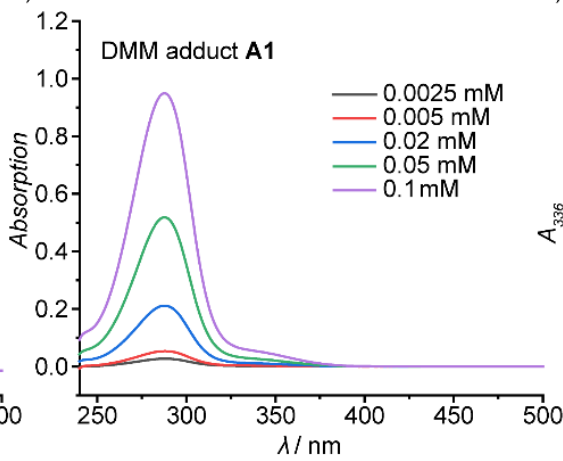

c)

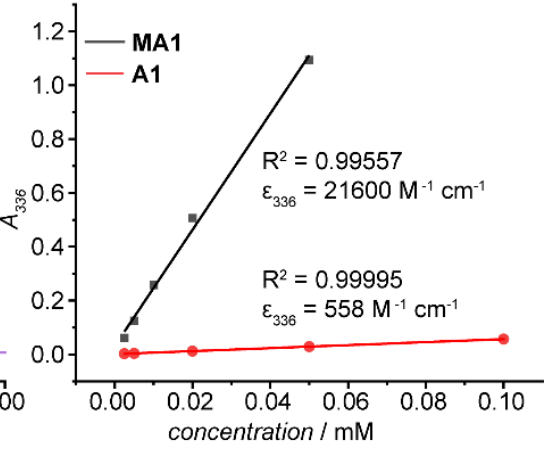

Figure S27. UV/Vis spectroscopy a) of Michael-acceptor MA1, c) the DMM adduct A1 at different concentrations and c) linear regression of the absorption of MA1 and $\mathbf{A 1}$ at $336 \mathrm{~nm}$. $\mathrm{H}_{2} \mathrm{O}$ :DMSO, 9:1, $20{ }^{\circ} \mathrm{C}$.

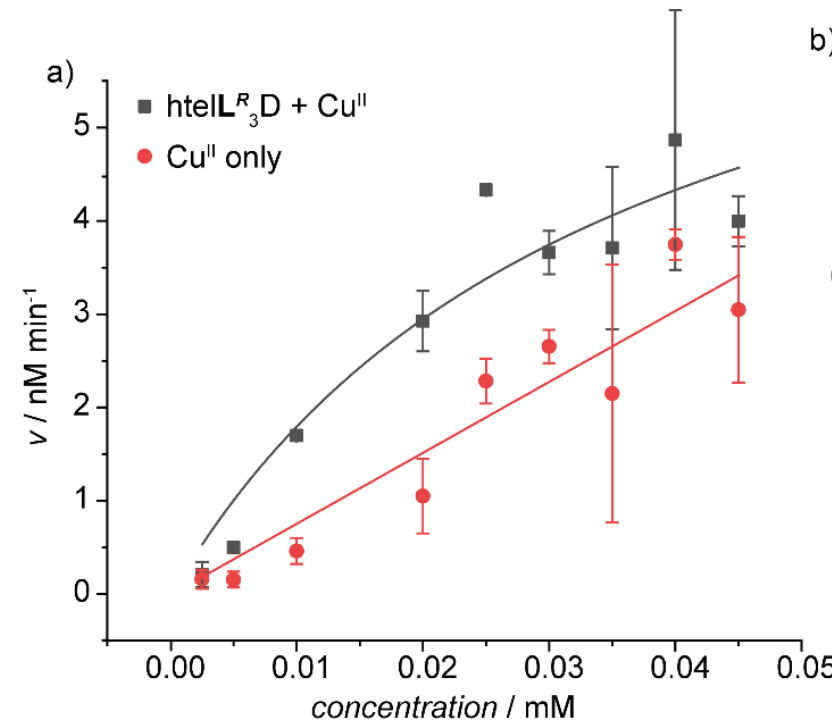

b) Model Michaelis-Menten

Equation $\quad y=V \max ^{*} x /(K m+x)$

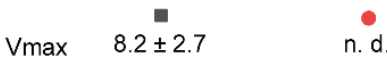

$\mathrm{Km} \quad 35.2 \mu \mathrm{M} \pm 25.7 \quad$ n. $\mathrm{d}$.

$\begin{array}{lll}R^{2} & 0.91 & 0.89\end{array}$

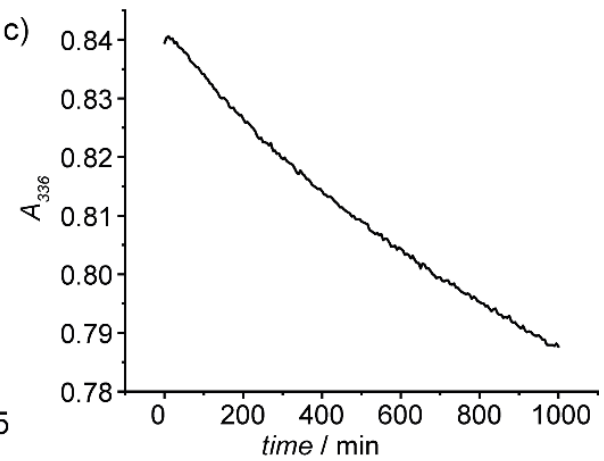

Figure S28. a) Michaelis-Menten saturation curve for the $\mathrm{Cu}(\mathrm{II})$-bound G-quadruplex htelL ${ }_{3}{ }_{3} \mathrm{D}$ (black curve) and b) corresponding fitting results obtained by the routine implemented in Origin 2019 (red curve: a linear fit was obtained for catalysis by free $\mathrm{Cu}(\mathrm{II})$, hence no Michaelis-Menten behaviour is observed in this case), c) exemplary substrate decay curve. Conditions: $1.2 \mu \mathrm{M}$ htelL ${ }_{3}{ }_{3} \mathrm{D}, 1 \mu \mathrm{M} \mathrm{CuSO}_{4}, 100 \mathrm{mM} \mathrm{KCl}, 10 \mathrm{mM} \mathrm{HEPES} \mathrm{pH}$ 8, $10 \% \mathrm{v} / \mathrm{v}$ DMSO, $25^{\circ} \mathrm{C}$. 


\section{UV/VIS and CD spectroscopy and thermal difference spectra}

All UV/Vis based measurements were carried out on Jasco V-650 and Jasco V-750 absorption spectrometers in quartz glass cuvettes. Samples were prepared with $2 \mu \mathrm{M}$ ssDNA in $100 \mathrm{mM} \mathrm{NaCl}$ or KCl, $10 \mathrm{mM}$ buffer and, if present, 2 $\mu \mathrm{M} \mathrm{CuSO}_{4}$. To form the G-quadruplexes, solutions were heated to $85^{\circ} \mathrm{C}$ and cooled down to $4{ }^{\circ} \mathrm{C}$ with $0.5{ }^{\circ} \mathrm{C} \mathrm{min}{ }^{-1}$ and left at $4{ }^{\circ} \mathrm{C}$ for several hours.

Thermal difference spectra (TDS) were recorded from 250 to $350 \mathrm{~nm}$ by subtracting the low temperature spectrum (0 $\left.{ }^{\circ} \mathrm{C}\right)$ from the high temperature spectrum $\left(85^{\circ} \mathrm{C}\right)$. The absorption at $350 \mathrm{~nm}$ was then set to 0 . The absorption spectra below $250 \mathrm{~nm}$ were dominated by the absorption of HEPES buffer.

Thermal denaturation experiments were carried out to determine the thermal stability expressed in the melting temperature $T_{\mathrm{m}}$. Therefore, the change in absorbance, as the temperature was increased with $0.5^{\circ} \mathrm{C} \mathrm{min}^{-1}$, was followed at $295 \mathrm{~nm}$ indicative for G-quadruplex denaturation and at $350 \mathrm{~nm}$ as control. The spectrometer bandwidth was set to $2 \mathrm{~nm}$. To control the temperature, a cuvette containing water was equipped with a temperature probe connected to the spectrometer. Water evaporation from the samples was avoided by addition of small amounts of silicon oil. To ensure equal heating rates between different experiments, always five samples were measured at a time. The measured absorbance was plotted against the temperature after the absorbance at $350 \mathrm{~nm}$ was subtracted to remove background absorption. Spectra were normalized to fraction folded values between 1 and 0 corresponding to fully folded or unfolded G-quadruplexes, respectively.

Table S11. Melting temperatures $T_{m}$ and thermal stabilizations $\left(\Delta T_{m}\right)$ of different G-quadruplex forming sequences with $\mathrm{KCl}$ or $\mathrm{NaCl}$ and in absence and presence of 1 equiv. $\mathrm{Cu}^{\mathrm{II}}$. Conditions: $2 \mu \mathrm{M}$ DNA, $2 \mu \mathrm{M} \mathrm{CuSO}_{4}, 100 \mathrm{mM} \mathrm{KCl} / \mathrm{NaCl}, 100 \mathrm{mM} \mathrm{TMAA} \mathrm{pH}$ 6.8, $\mathrm{H}_{2} \mathrm{O}: \mathrm{ACN}, 1: 1$.

\begin{tabular}{|c|c|c|c|c|}
\hline Sequence & $\mathrm{NaCl}$ & $\mathrm{NaCl}+\mathrm{Cu}^{\mathrm{II}}$ & KCl & $\mathrm{KCl}+\mathrm{Cu}^{\mathrm{II}}$ \\
\hline htelL ${ }^{S_{3} \mathrm{D}}$ & 46 & $55(+9)$ & 62 & $71(+9)$ \\
\hline htell $L_{3} S_{3}$ & 48 & $54(+6)$ & 62 & $66(+4)$ \\
\hline htel $\mathbf{L}_{2} \mathrm{~F}$ & 52 & $52(+0)$ & 68 & $67(-1)$ \\
\hline htelL $\mathbf{L}_{2} \mathrm{G}$ & 46 & $46(+0)$ & 59 & $59(+0)$ \\
\hline htel $\mathbf{L}^{R_{3} \mathrm{D}}$ & 46 & $57(+11)$ & 63 & $68(+5)$ \\
\hline htel $\mathbf{L}^{R}{ }_{3} \mathrm{H}$ & 53 & $56(+3)$ & 68 & $68(+0)$ \\
\hline htel $\mathbf{L}^{R_{2}} \mathrm{~F}$ & 49 & $50(+1)$ & 67 & $66(-1)$ \\
\hline htel $\mathbf{L}^{\boldsymbol{R}_{2} \mathrm{G}}$ & 47 & $47(0)$ & 66 & $66(+0)$ \\
\hline
\end{tabular}

$\mathrm{CD}$ measurements were carried out on a Chirascan $q C D$ spectrometer in black quartz cuvettes. Samples were prepared as described before. Temperature was controlled using a Quantum Northwest temperature control attached to a sample probe. Spectra were recorded from 220 to $350 \mathrm{~nm}\left(120 \mathrm{~nm} \mathrm{~min}^{-1}\right)$ with a $1 \mathrm{~nm}$ interval and $0.5 \mathrm{~nm}$ bandwidth three times and averaged using the built-in software. The averaged spectra were smoothed (Savitzky-Golay) with a factor of 5 and the smoothed background (sample containing only buffer, smooth factor 10) was subtracted. The background was recorded once from 220 to $350 \mathrm{~nm}$ with the same settings as for the samples in the same cuvette. 

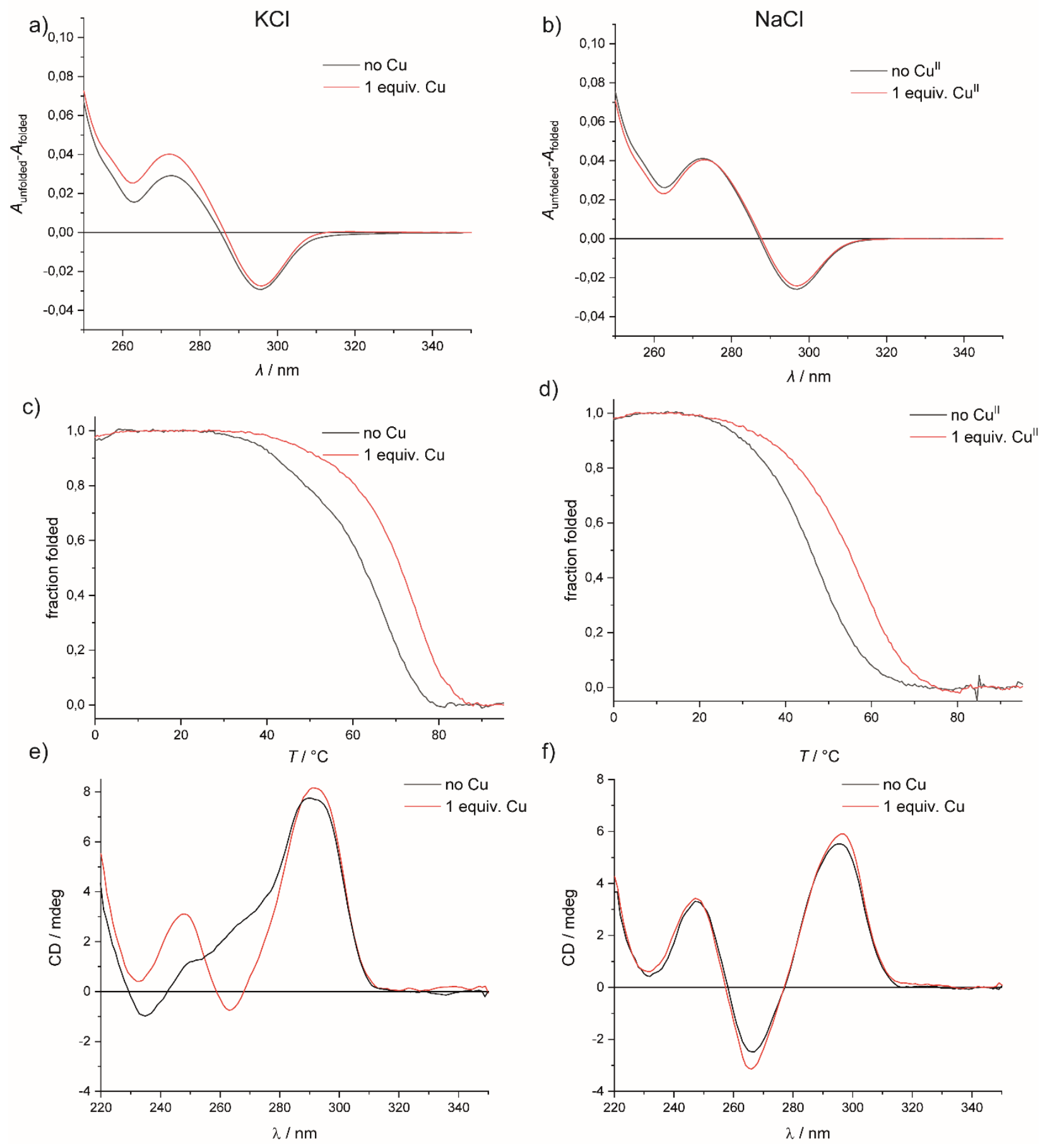

Figure S29. TDS (a, b), Thermal denaturation (c, d) and CD (e, f) spectra of htelL $S_{3}$ D using potassium chloride (a), c) and e)) and sodium chloride (b), d) and f)) as electrolyte. Conditions: $2 \mu \mathrm{M}$ DNA, $10 \mathrm{mM}$ HEPES pH 8, $100 \mathrm{mM}$ electrolyte and if used $2 \mu \mathrm{M}$ $\mathrm{CuSO}_{4}$. 

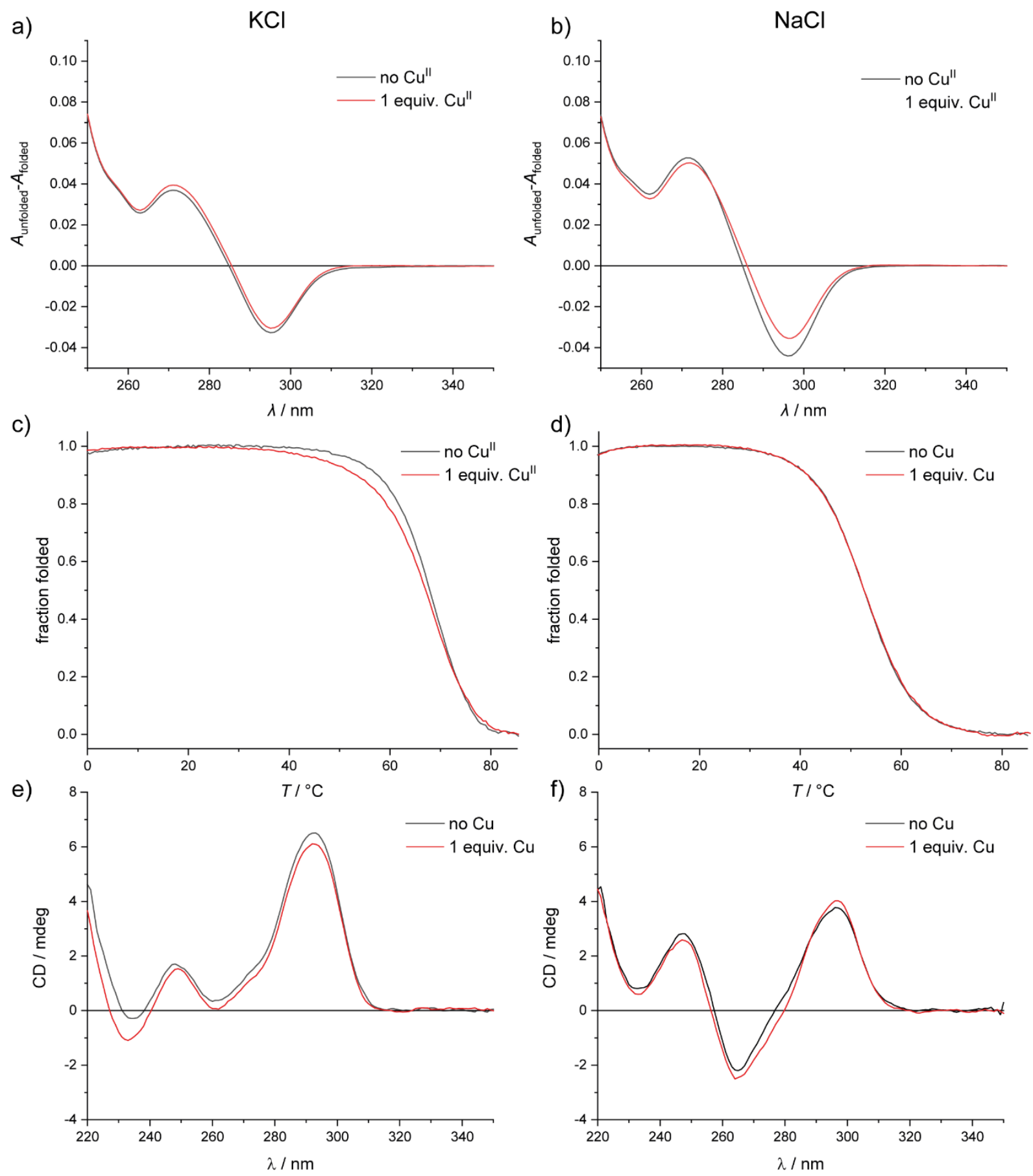

Figure S30. TDS (a, b), Thermal denaturation (c, d) and CD (e, f) spectra of htelL $S_{2}$ F using potassium chloride (a), c) and e)) and sodium chloride (b), d) and f)) as electrolyte. Conditions: $2 \mu \mathrm{M}$ DNA, $10 \mathrm{mM}$ HEPES pH 8, $100 \mathrm{mM}$ electrolyte and if used $2 \mu \mathrm{M}$ $\mathrm{CuSO}_{4}$. 


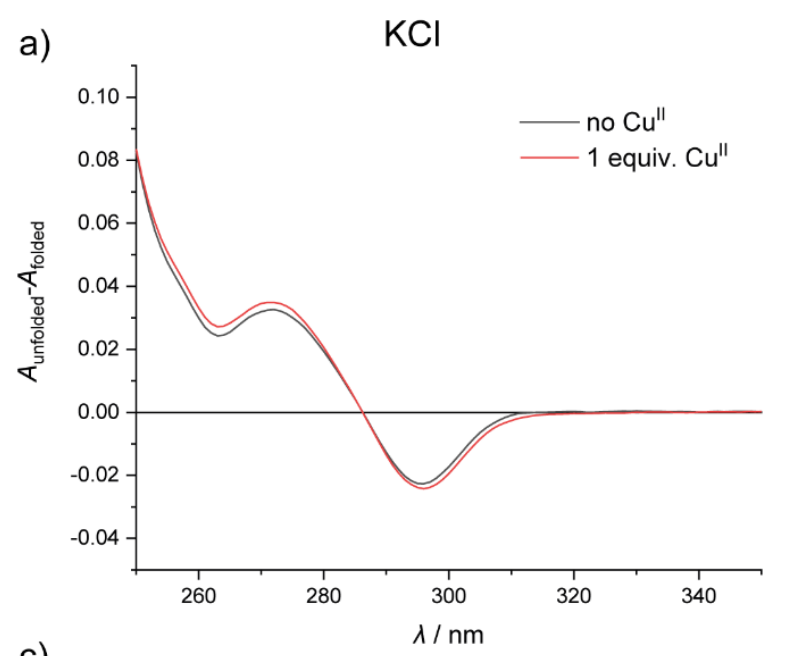

c)
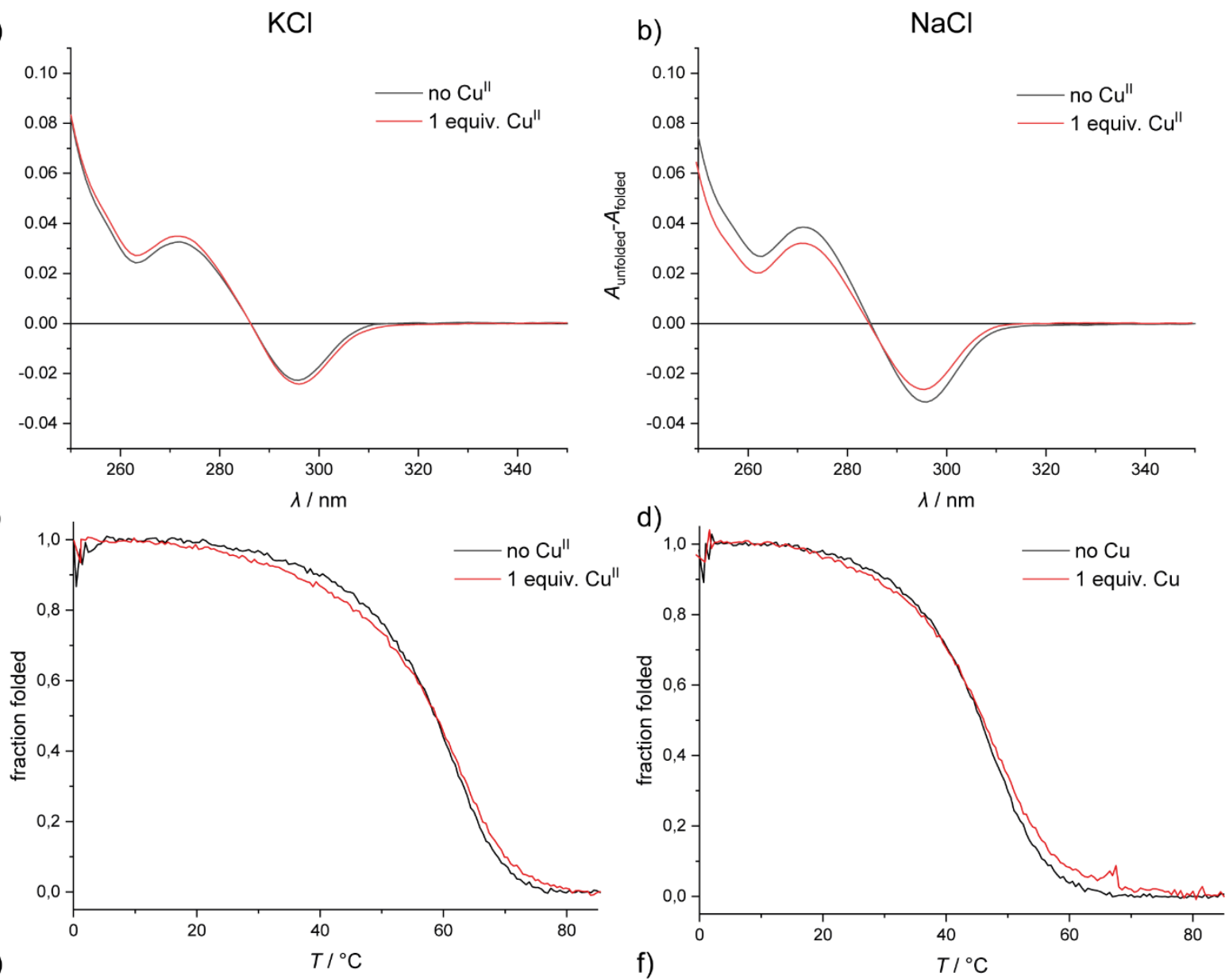

e)
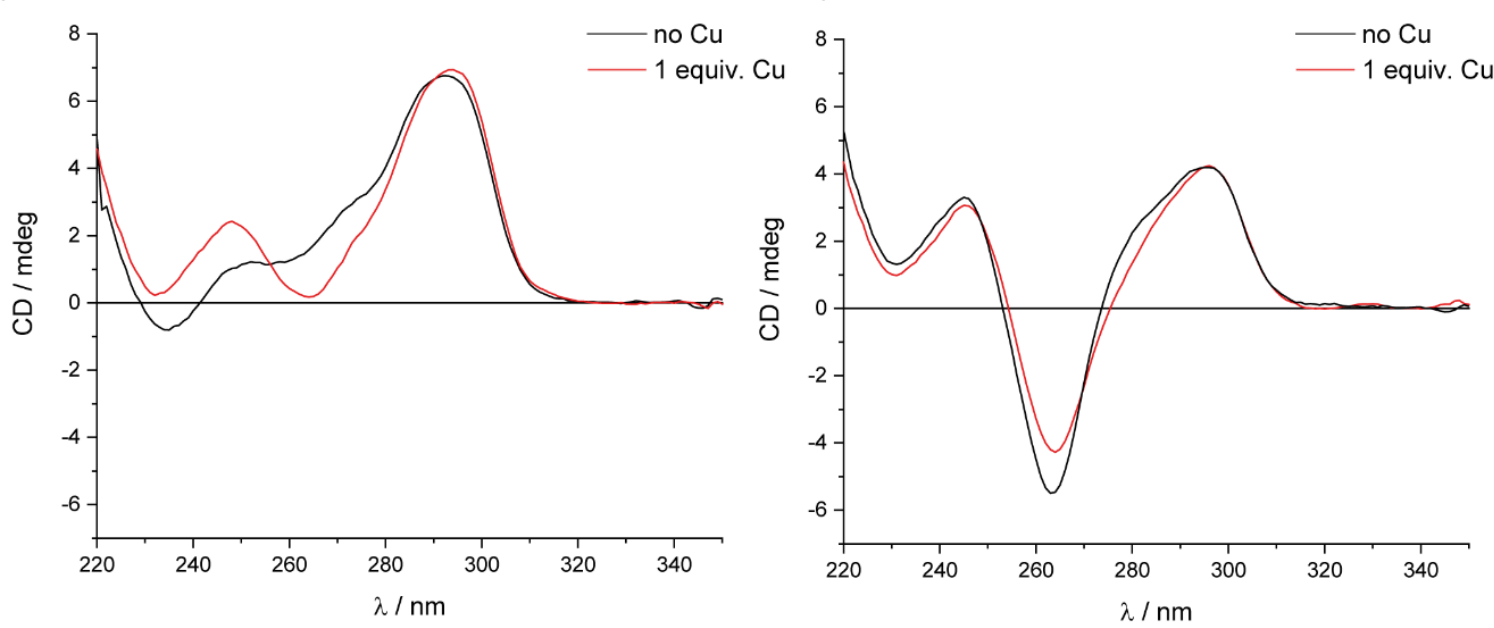

Figure S31. TDS (a, b), Thermal denaturation (c, d) and CD (e, f) spectra of htel $\boldsymbol{S}_{2} \mathrm{G}$ using potassium chloride (a), c) and e)) and sodium chloride (b), d) and f)) as electrolyte. Conditions: $2 \mu \mathrm{M}$ DNA, $10 \mathrm{mM}$ HEPES pH 8, $100 \mathrm{mM}$ electrolyte and if used $2 \mu \mathrm{M}$ $\mathrm{CuSO}_{4}$. 

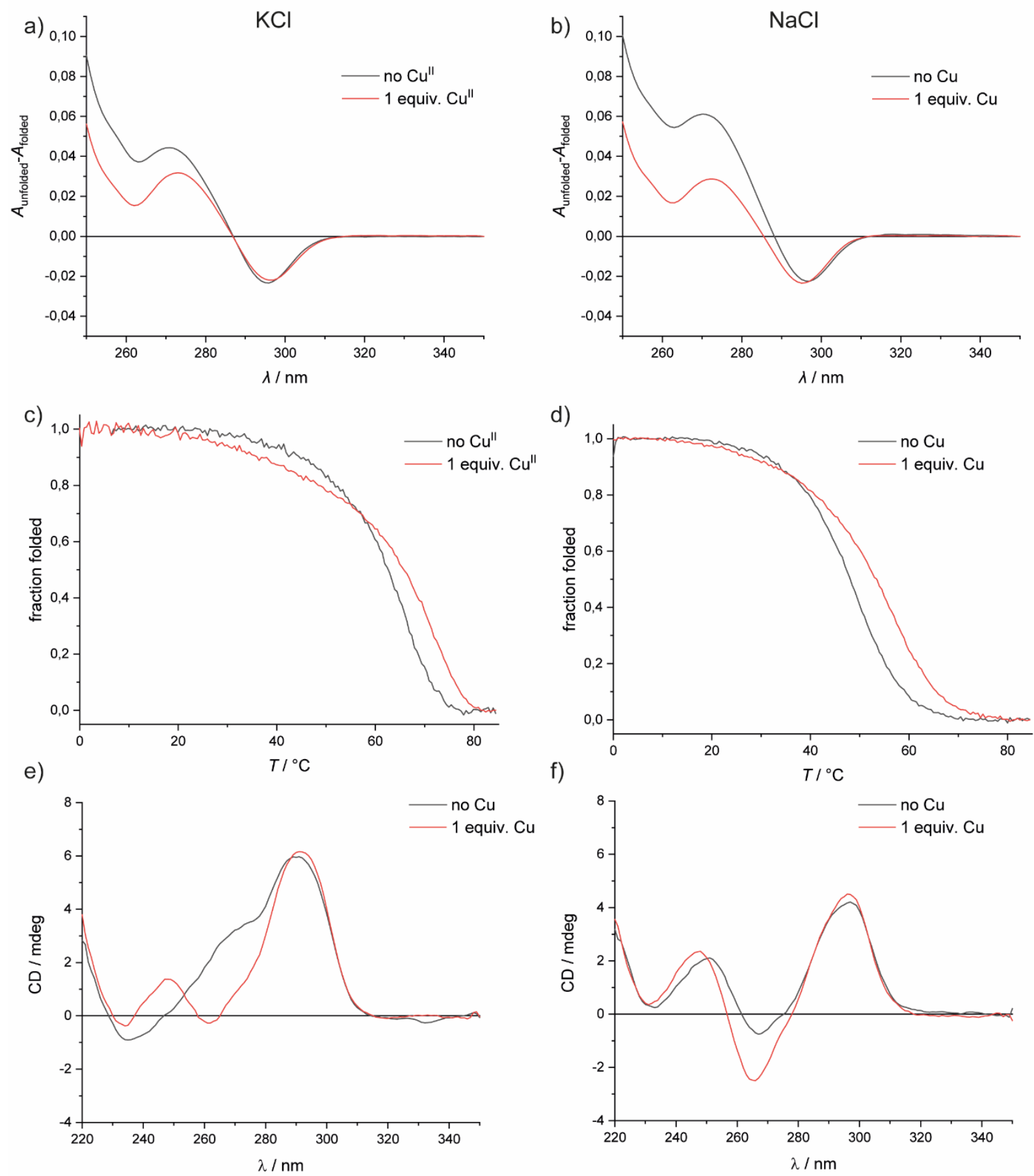

Figure S32. TDS (a, b), Thermal denaturation (c, d) and CD (e, f) spectra of htelL $S_{3} \mathrm{~B}$ using potassium chloride (a), c) and e)) and sodium chloride (b), d) and f)) as electrolyte. Conditions: $2 \mu \mathrm{M}$ DNA, $10 \mathrm{mM}$ HEPES pH 8, $100 \mathrm{mM}$ electrolyte and if used $2 \mu \mathrm{M}$ $\mathrm{CuSO}_{4}$. 
$\mathrm{KCl}$
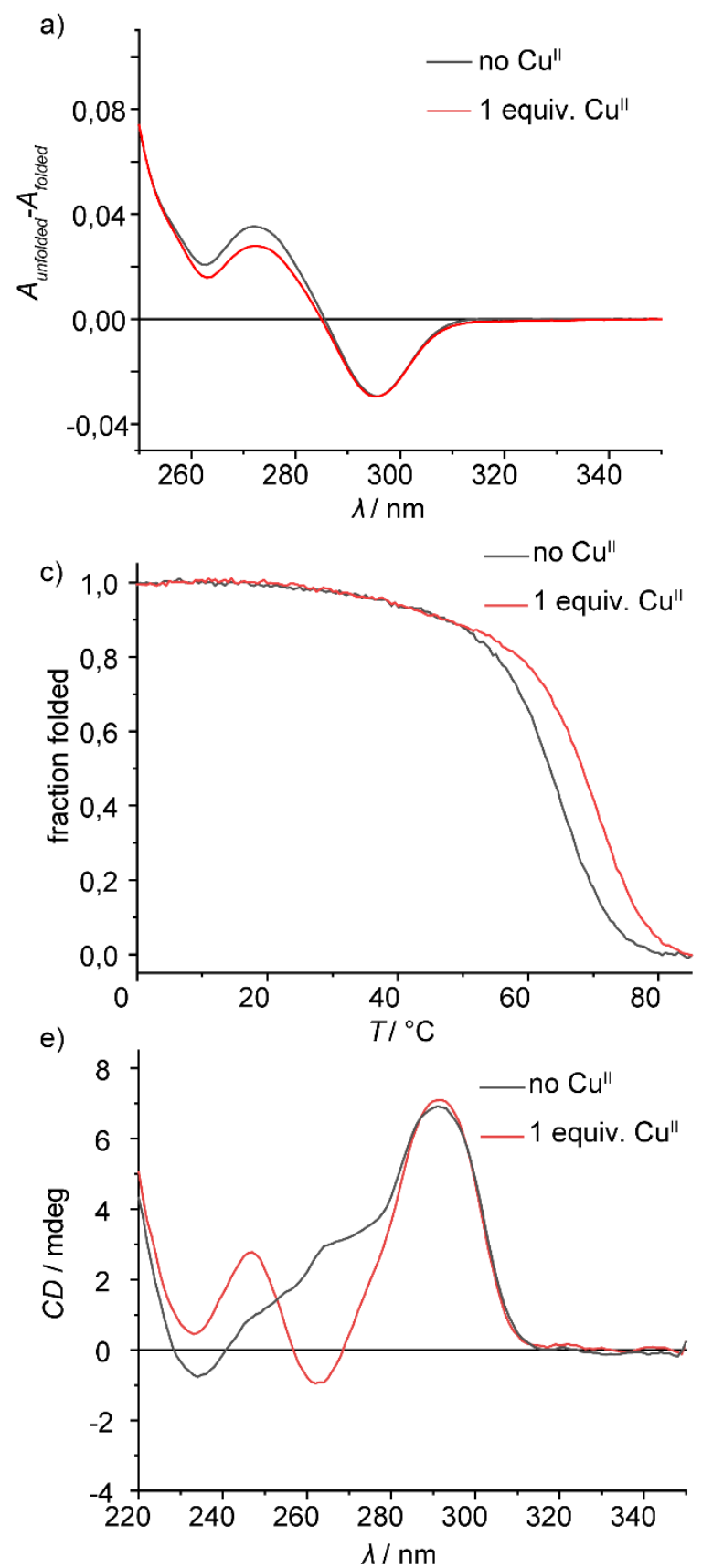

$\mathrm{NaCl}$
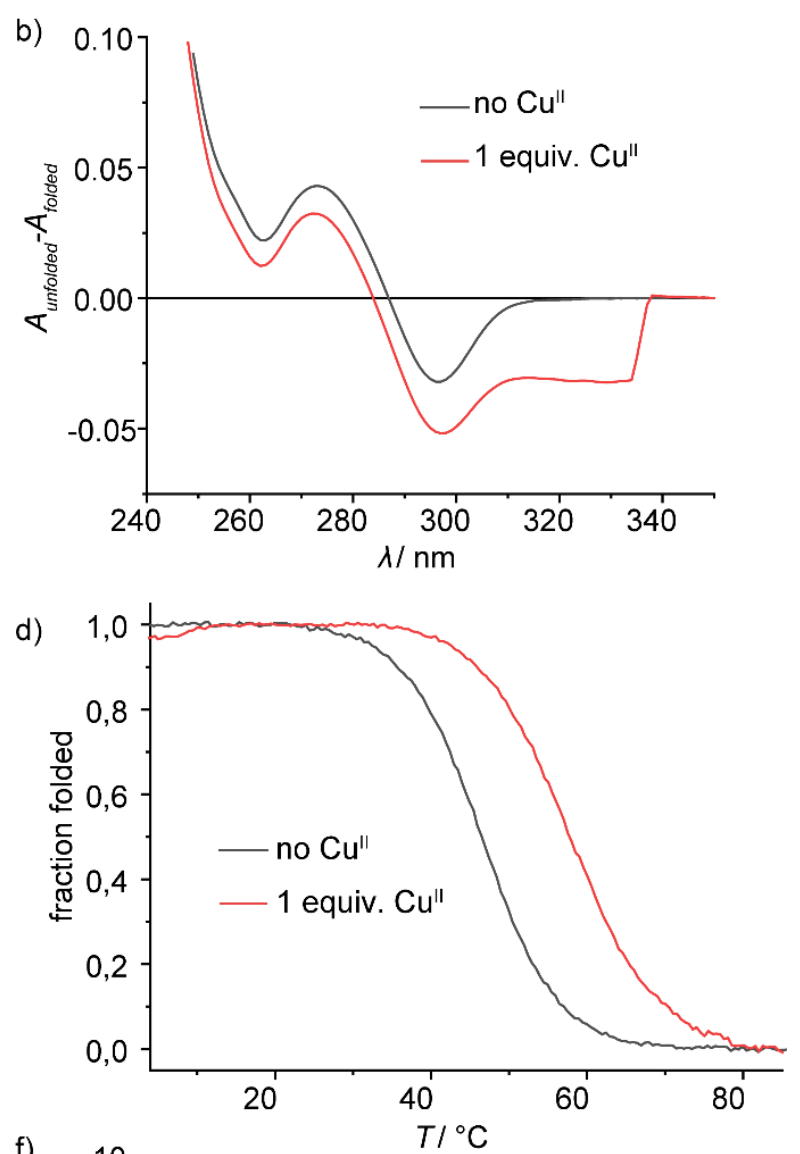

f)

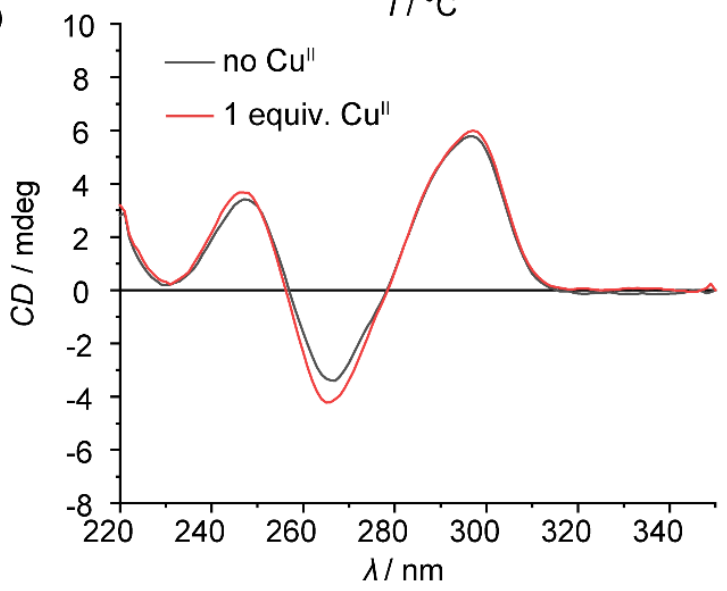

Figure S33. TDS (a, b), Thermal denaturation (c, d) and CD (e, f) spectra of htelL ${ }^{R_{3}} \mathrm{D}$ using potassium chloride $(\mathrm{a}, \mathrm{c}$ and $\mathrm{e})$ and sodium chloride (b, $d$ and f) as electrolyte. Conditions: $2 \mu \mathrm{M}$ DNA, $10 \mathrm{mM}$ HEPES pH 8, $100 \mathrm{mM}$ electrolyte and if used $2 \mu \mathrm{M}$ $\mathrm{CuSO}_{4}$. 
$\mathrm{KCl}$
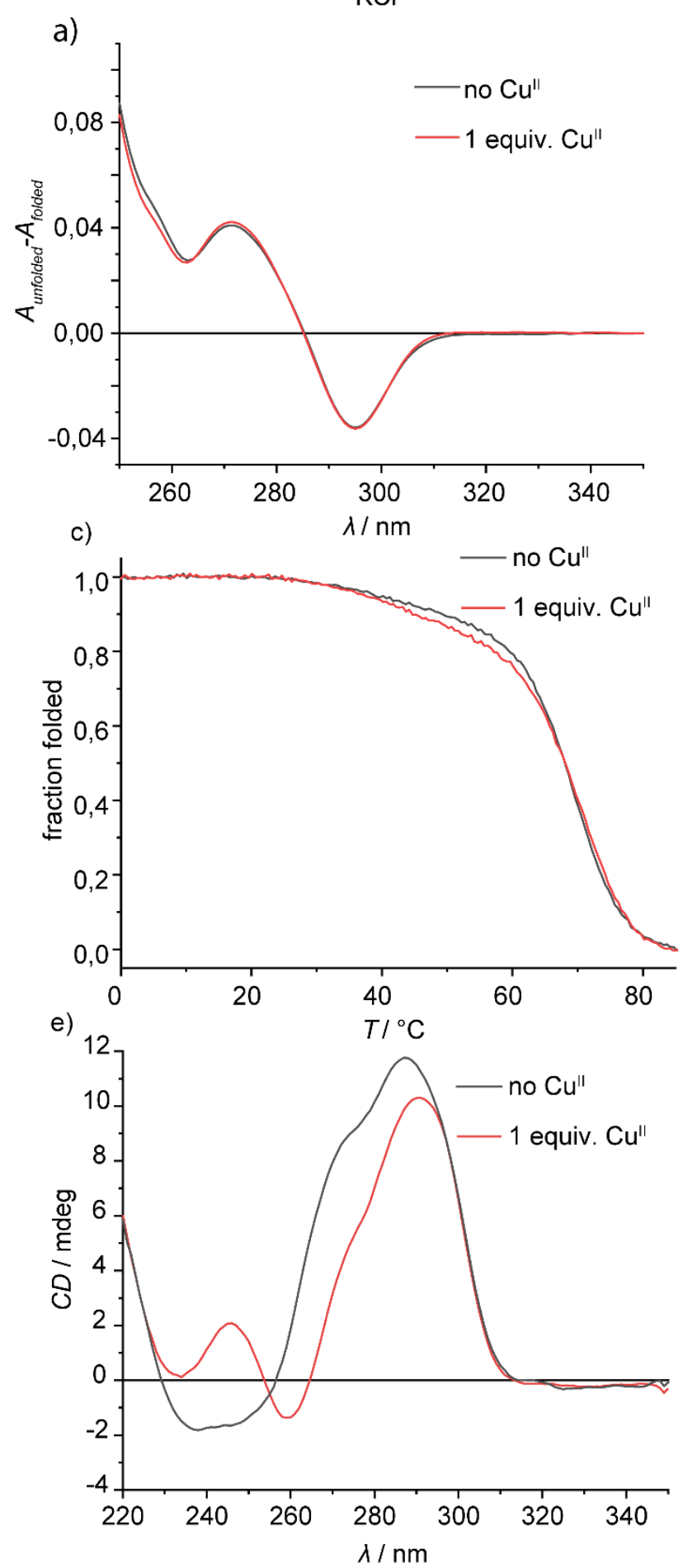
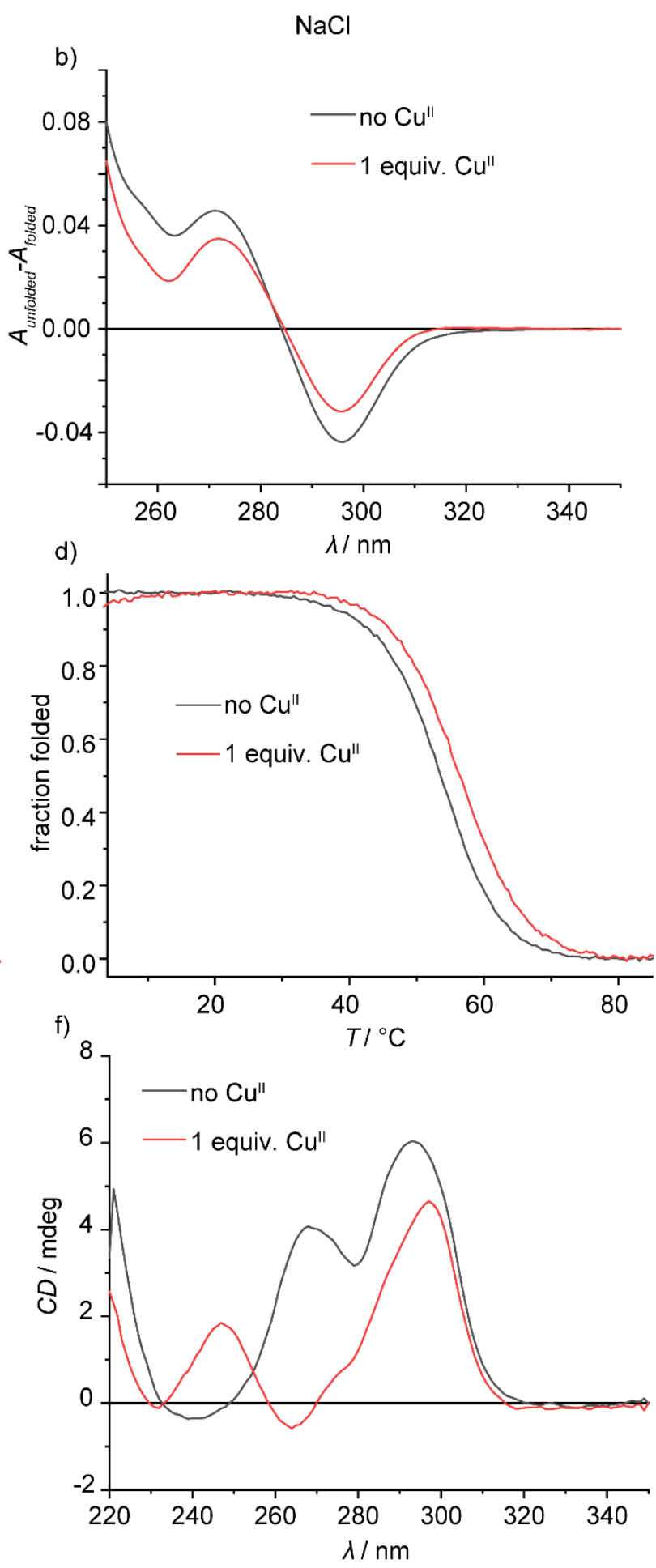

Figure S34. TDS (a, b), Thermal denaturation (c, d) and CD (e, f) spectra of htel $\mathbf{L}^{\boldsymbol{R}}{ }_{3} \mathrm{H}$ using potassium chloride (a, c and e) and sodium chloride (b, $\mathrm{d}$ and $\mathrm{f}$ ) as electrolyte. Conditions: $2 \mu \mathrm{M}$ DNA, $10 \mathrm{mM}$ HEPES pH 8, $100 \mathrm{mM}$ electrolyte and if used $2 \mu \mathrm{M}$ $\mathrm{CuSO}_{4}$. 
$\mathrm{KCl}$

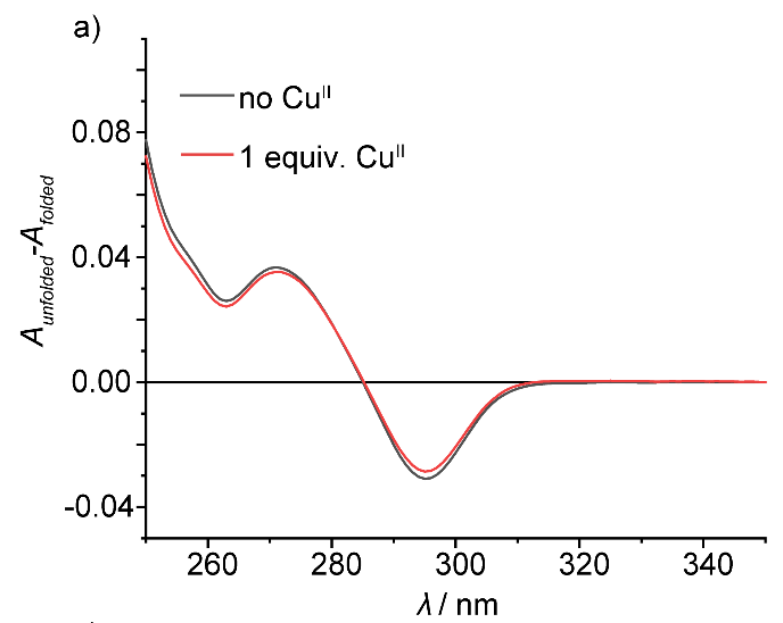

$$
\text { c) }
$$
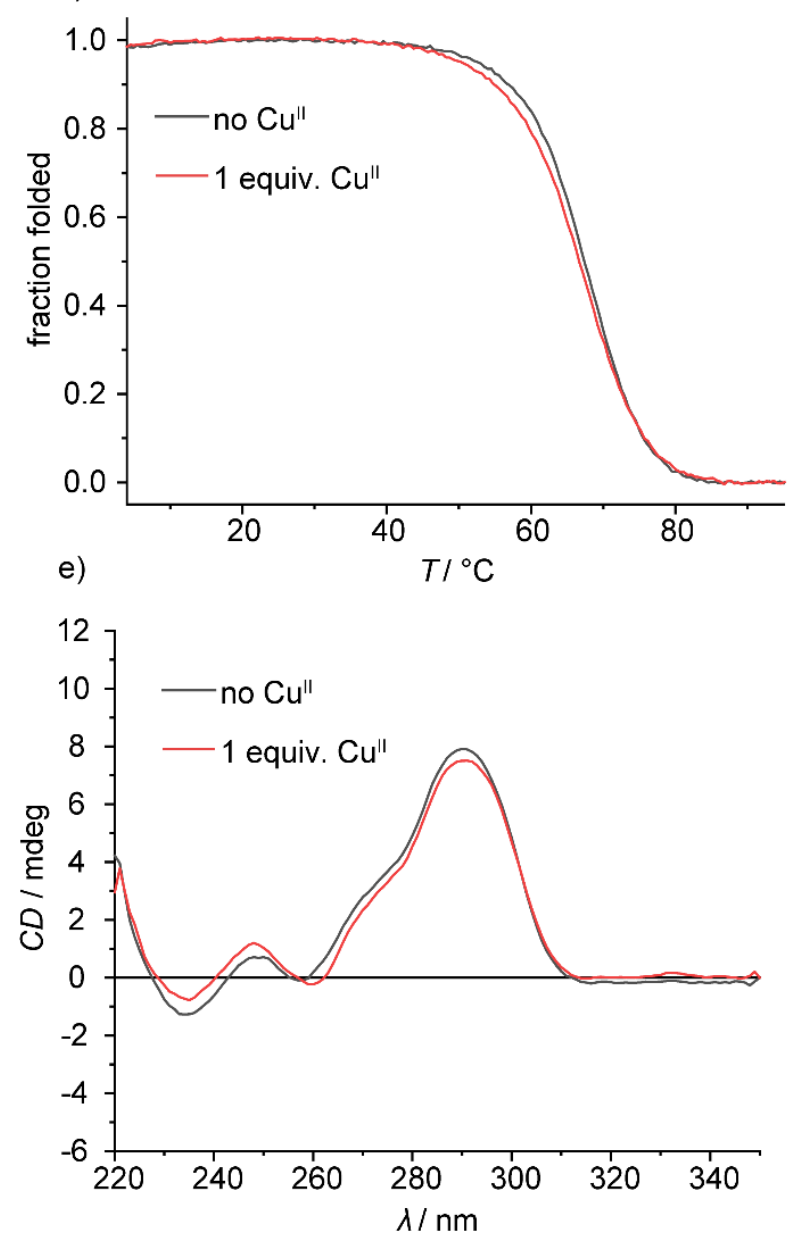
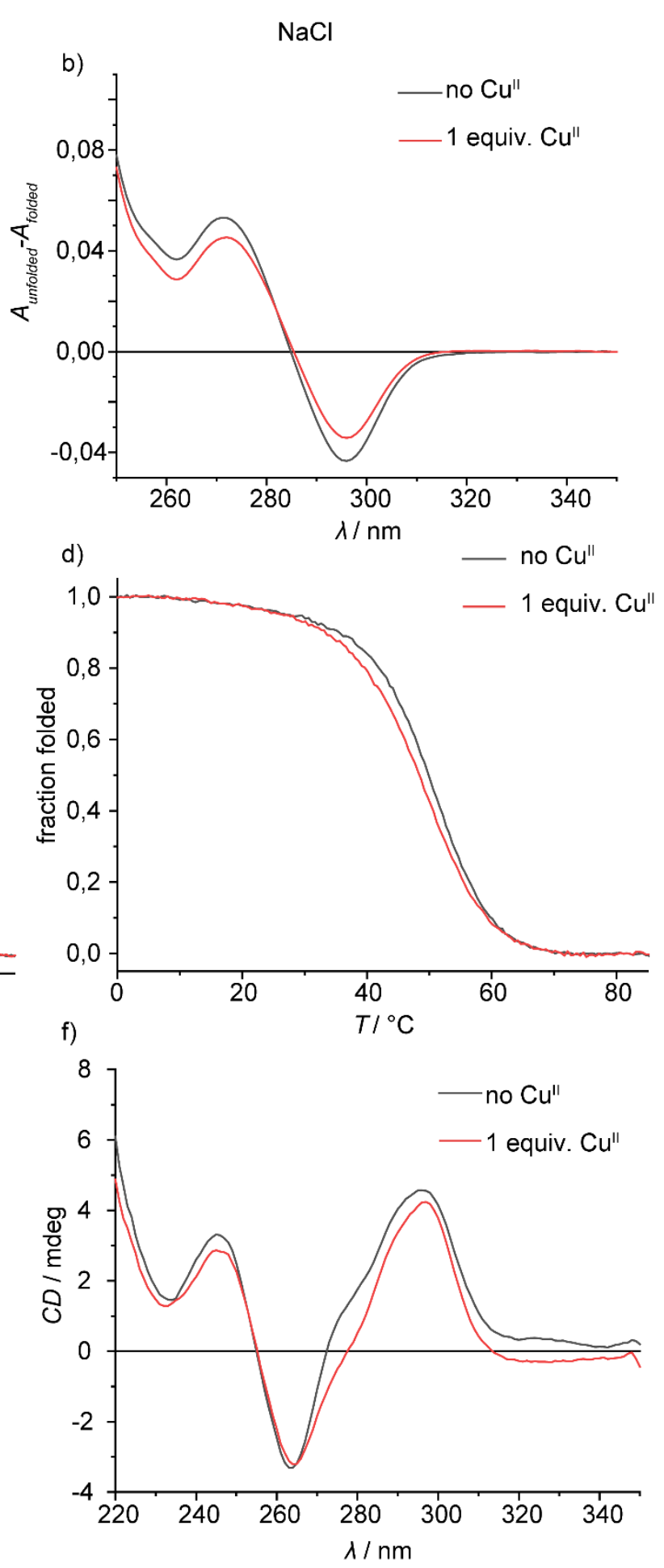

Figure S35. TDS (a, b), Thermal denaturation (c, d) and CD (e, f) spectra of htel $\mathbf{L}^{\boldsymbol{R}}{ }_{2} \mathrm{~F}$ using potassium chloride (a, c and e) and sodium chloride (b, $\mathrm{d}$ and $\mathrm{f}$ ) as electrolyte. Conditions: $2 \mu \mathrm{M}$ DNA, $10 \mathrm{mM}$ HEPES pH 8, $100 \mathrm{mM}$ electrolyte and if used $2 \mu \mathrm{M}$ $\mathrm{CuSO}_{4}$ 

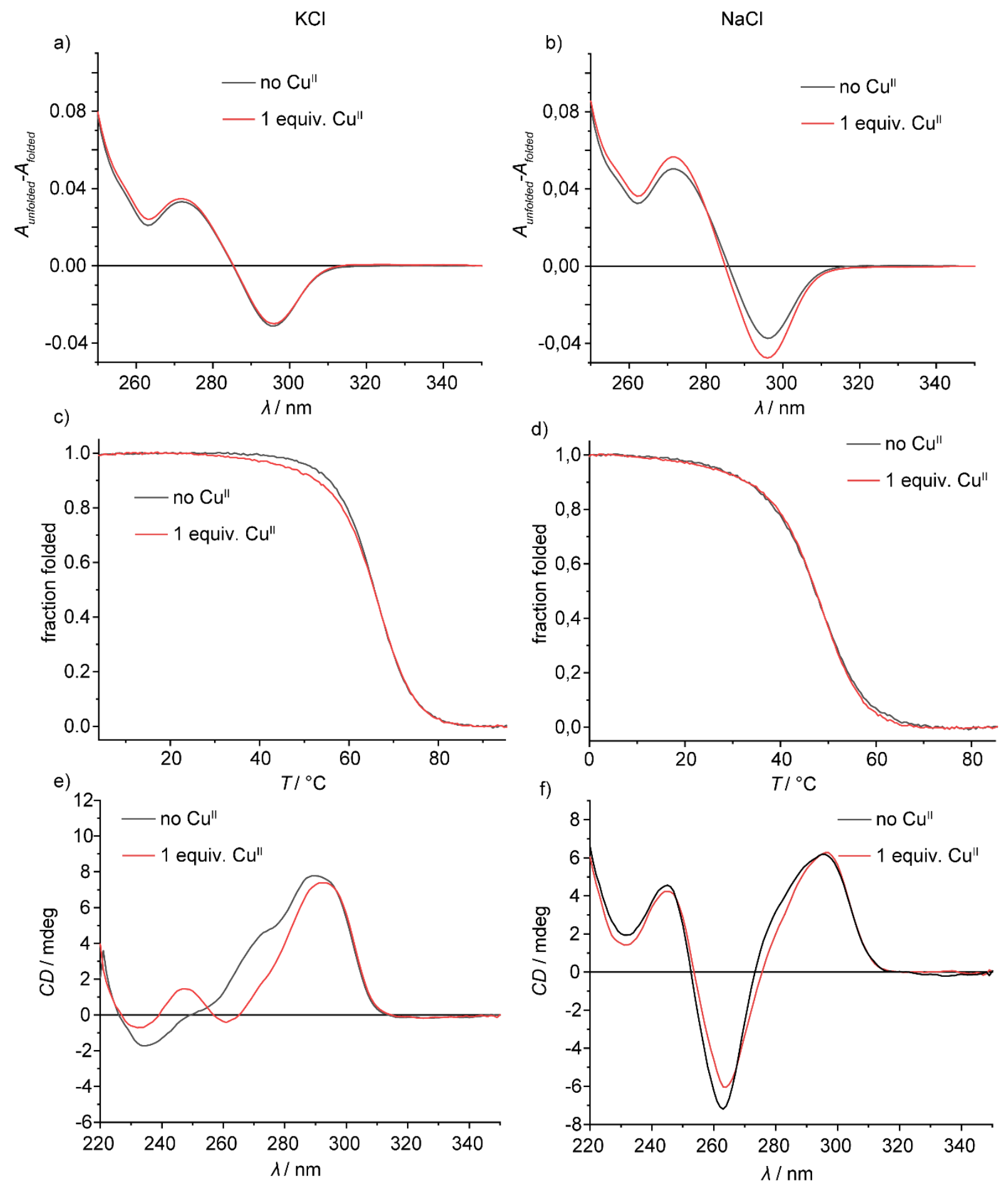

Figure S36. TDS (a, b), Thermal denaturation (c, d) and CD (e, f) spectra of htel $\mathbf{L}^{\boldsymbol{R}_{2}} \mathrm{G}$ using potassium chloride (a, c and e) and sodium chloride (b, $\mathrm{d}$ and $\mathrm{f}$ ) as electrolyte. Conditions: $2 \mu \mathrm{M}$ DNA, $10 \mathrm{mM}$ HEPES pH 8, $100 \mathrm{mM}$ electrolyte and if used $2 \mu \mathrm{M}$ $\mathrm{CuSO}_{4}$ 

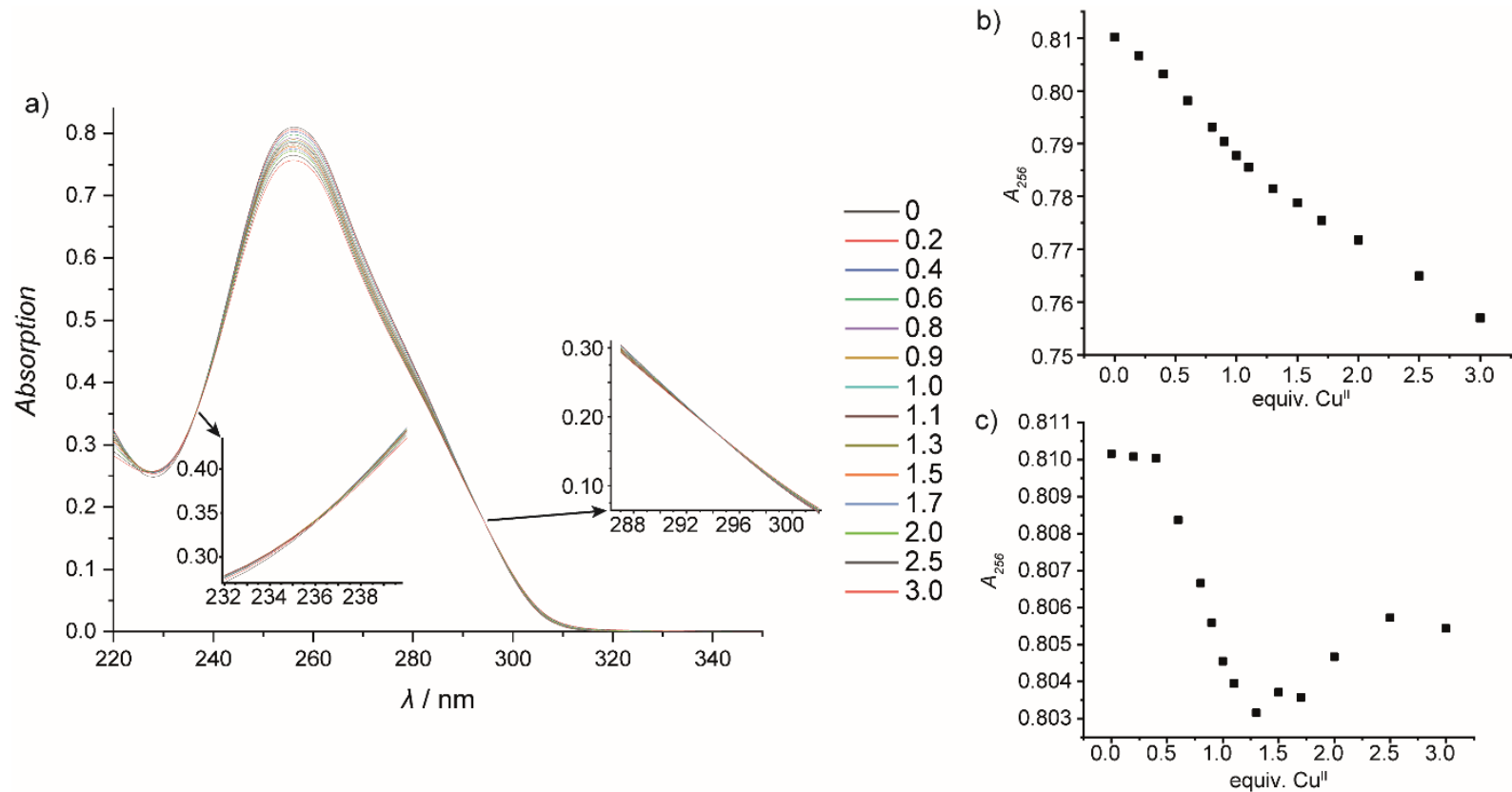

Figure S37. $\mathrm{Cu}^{\mathrm{II}}$ titration to htel $\mathbf{L}^{R_{3}} \mathrm{D}$. a) A UV spectrum was recorded after every $\mathrm{Cu}^{\mathrm{II}}$ addition. The insets show the isosbestic points. The absorption at $256 \mathrm{~nm}$ was plotted against the $\mathrm{Cu}^{\mathrm{II}}$ equivalents in b) with the uncorrected absorption and c) after correcting for dilution. $4 \mu \mathrm{M}$ DNA, $10 \mathrm{mM}$ HEPES $\mathrm{pH} 8,100 \mathrm{mM} \mathrm{KCl}$ and $\mathrm{CuSO}_{4}, 25^{\circ} \mathrm{C}$.

\section{Native ESI-MS}

Native ESI mass spectrometry was performed on a Bruker ESI-timsTOF mass spectrometer (negative mode, capillary voltage: $4500 \mathrm{~V}$, end plate offset voltage: $500 \mathrm{~V}$, nebulizer gas pressure: $0.4 / 0.6$ bar, dry gas flow rate: $6 / 9 \mathrm{~L} / \mathrm{min}$, dry temperature: 303 K). For calibration of the TOF device, Agilent ESI-Low Concentration Tuning Mix was used.

In native ESI-MS, the secondary structure is kept intact. To differentiate between folded and unfolded G-quadruplexes in the gas phase, two phenomena are most instructive. First, if tetramolecular G-quadruplexes are investigated and the structure is denatured, single-stranded DNA instead of a tetramer would be observed and second, valid for tetra- and unimolecular G-quadruplexes, ESI mass spectrometry from electrolyte-containing solutions always gives rise to series of unspecific adducts with sodium or potassium cations. For fully denatured species, a statistical distribution of adducts starting with zero cations would be observed and for a native, folded species a distribution is observed starting with n1 explicitly bound cations where $\mathrm{n}$ is the number of G-tetrads. ${ }^{[10,11]}$ Samples containing $25 \mu \mathrm{M}$ DNA, 25 or $30 \mu \mathrm{M}$ $\mathrm{CuSO}_{4}, 1 \mathrm{mM} \mathrm{KCl}$ and $100 \mathrm{mM}$ TMAA pH 6.8 were annealed by heating them to $85^{\circ} \mathrm{C}$ and cooling them down to 4 ${ }^{\circ} \mathrm{C}$ with $0.5{ }^{\circ} \mathrm{C} \mathrm{min}^{-1}$. After annealing samples were diluted $1: 1$ with acetonitrile resulting in a final concentration of $12.5 \mu \mathrm{M}$ DNA, 12.5 or $15 \mu \mathrm{M} \mathrm{CuSO}_{4}, 0.5 \mathrm{mM} \mathrm{KCl}$ and $50 \mathrm{mM}$ TMAA $\mathrm{pH} 6.8$. 


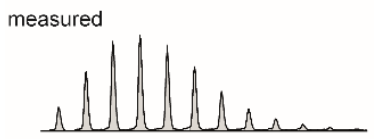

calculated for: $\mathrm{C}_{214} \mathrm{H}_{267} \mathrm{~N}_{86} \mathrm{O}_{130} \mathrm{P}_{21} \mathrm{~K}_{2}$

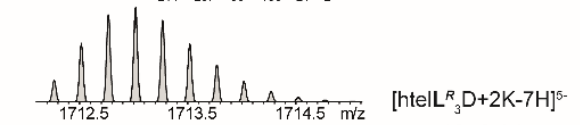

[htelL $\left.{ }^{R} \mathrm{D}+2 \mathrm{~K}-6 \mathrm{H}\right]^{4-}$

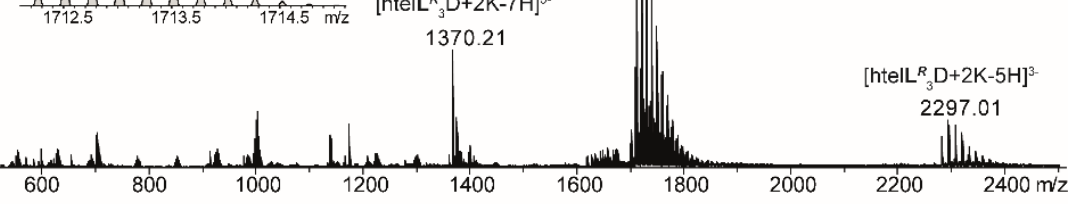

b)

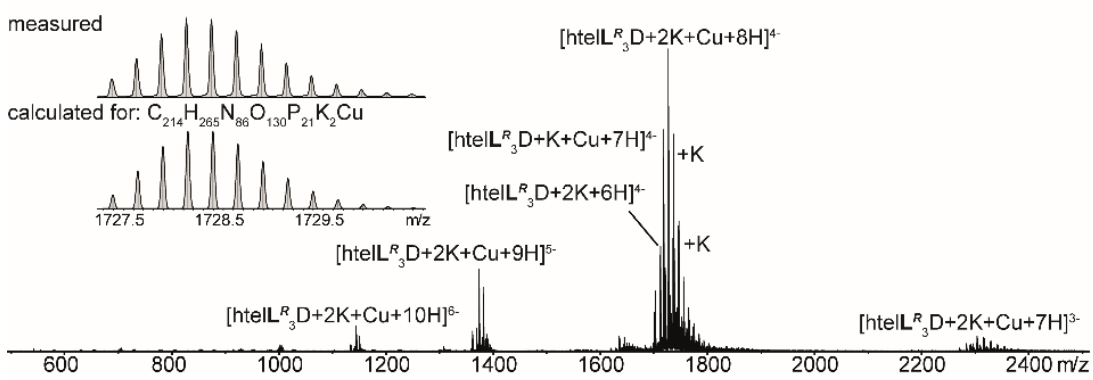

Figure S38. Native ESI-MS of htel: ${ }^{R_{3}} \mathrm{D}$ in a) absence and b) presence of $\mathrm{Cu}^{\mathrm{II}}$. Conditions: $12.5 \mu \mathrm{M}$ DNA, $15 \mu \mathrm{M} \mathrm{CuSO}, 0.5 \mathrm{mM}$ $\mathrm{KCl}, 50$ mM TMAA pH 6.8, $\mathrm{H}_{2} \mathrm{O}: \mathrm{ACN}, 1: 1$.

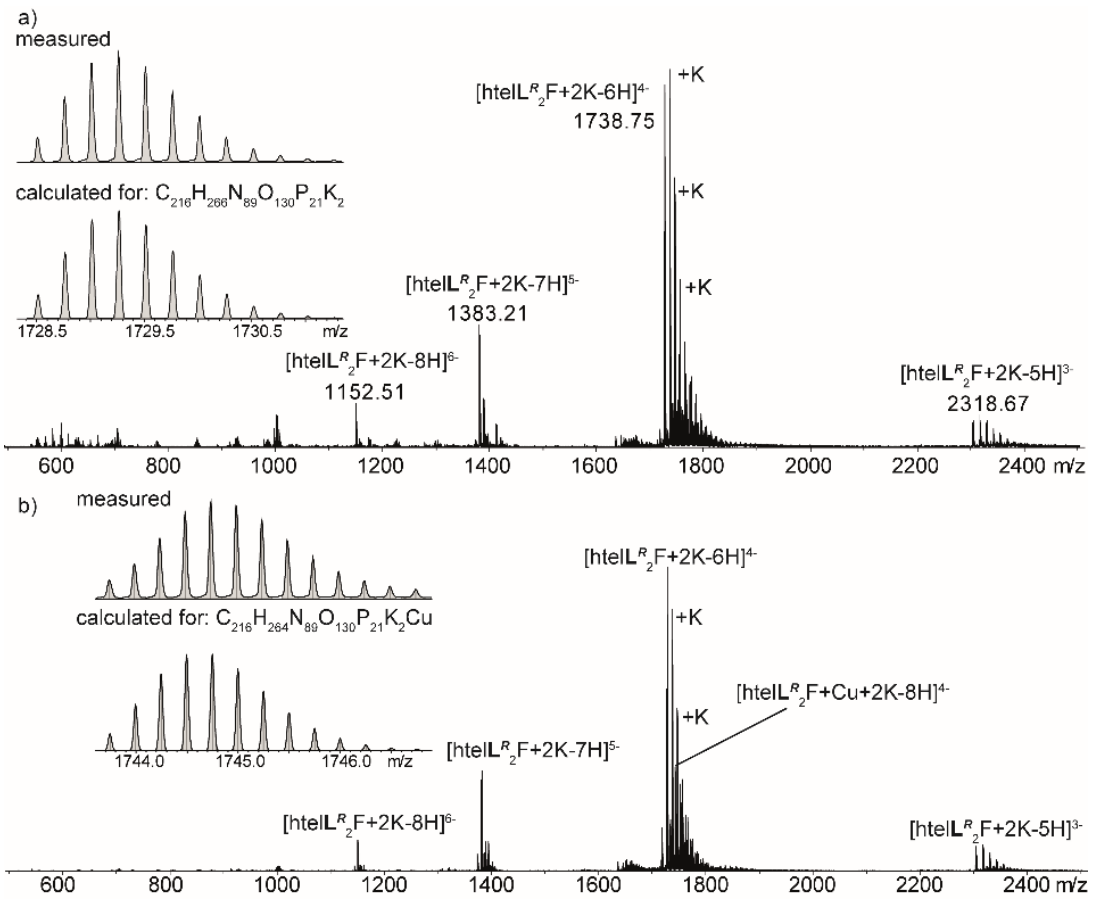

Figure S39. Native ESI-MS of htel: ${ }^{\boldsymbol{R}_{2} \mathrm{~F}}$ in a) absence and b) presence of $\mathrm{Cu}^{\mathrm{II}}$. Conditions: $12.5 \mu \mathrm{M}$ DNA, $15 \mu \mathrm{M} \mathrm{CuSO}, 0.5 \mathrm{mM}$ $\mathrm{KCl}, 50$ mM TMAA pH 6.8, $\mathrm{H}_{2} \mathrm{O}: \mathrm{ACN}, 1: 1$. 
a)
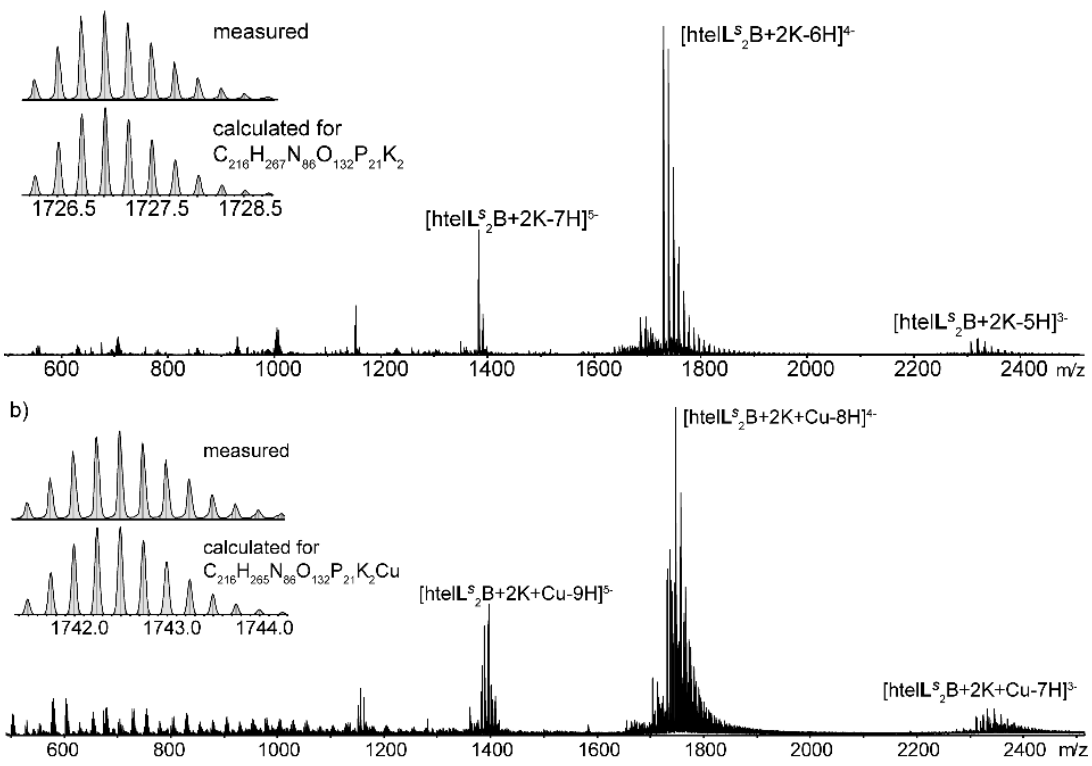

Figure S40. Native ESI-MS of htel $\mathrm{L}_{2}{ }_{2} \mathrm{~B}$ in a) absence and b) presence of $\mathrm{Cu}^{\mathrm{II}}$. Conditions: $12.5 \mu \mathrm{M}$ DNA, $12.5 \mu \mathrm{M} \mathrm{CuSO}, 0.5$ $\mathrm{mM} \mathrm{KCl}, 50 \mathrm{mM}$ TMAA pH 6.8, $\mathrm{H}_{2} \mathrm{O}: \mathrm{ACN}, 1: 1$.

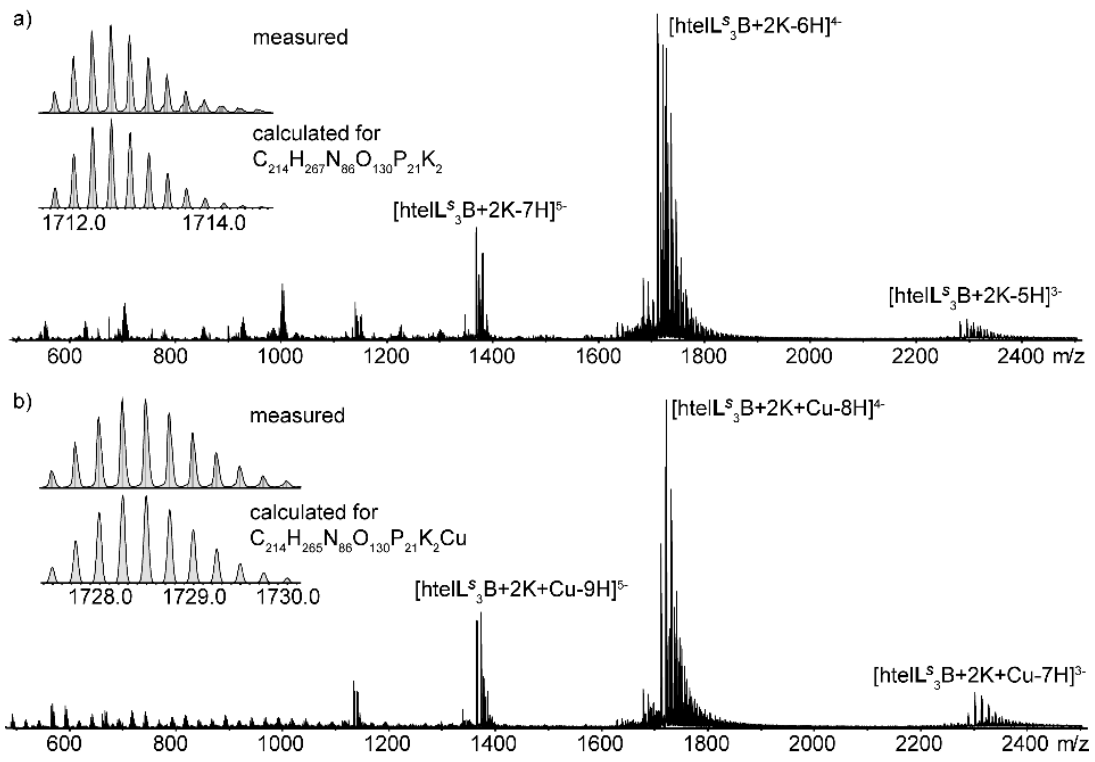

Figure S41. Native ESI-MS of htel $\mathbf{L}_{3} \mathrm{~B}$ in a) absence and b) presence of $\mathrm{Cu}^{\mathrm{II}}$. Conditions: $12.5 \mu \mathrm{M} \mathrm{DNA}, 12.5 \mu \mathrm{M} \mathrm{CuSO}_{4}, 0.5$ mM KCl, 50 mM TMAA pH 6.8, $\mathrm{H}_{2} \mathrm{O}: \mathrm{ACN}, 1: 1$.

When comparing the ESI mass spectra recorded for htel $\mathbf{L}^{R} \mathrm{D}$ and htel $\mathbf{L}^{R_{2}} \mathrm{~F}$ in absence or presence of $\mathrm{Cu}^{\mathrm{II}}$ cations, it can be seen that the former sequence featuring three ligands seems to form a more stable $\mathrm{Cu}$-complex (highest peak in adduct distribution), while the latter G-quadruplex affords the $\mathrm{Cu}$-free DNA peak as highest signal even in presence of $\mathrm{Cu}^{\mathrm{II}}$ cations. We see the reason for this observation in the unfavourable positioning of only two contained ligands in the same loop. For htelL ${ }_{2}{ }_{2} \mathrm{~B}$, however, also featuring two ligands, the signal corresponding to $\mathrm{Cu} @$ htel $\mathrm{L}^{S_{2} \mathrm{~B}}$ was observed to show about as high intensity as its three-ligand relative htel $\mathbf{L}_{3} \mathrm{~B}$, carrying three ligands. The reason for these differing stabilities might be the positioning of the ligandosides within the loops: While in htel $\mathbf{L}_{2}{ }_{2} \mathrm{~F}$ both ligandosides were incorporated next to each other in loop 1 , for $h_{t e l} \mathbf{L}_{2} \mathrm{~B}$ the ligandosides were incorporated in the oppositely arranged loops 1 and 3 . This might result in a more favorable coordination geometry and hence a more stable complexation of $\mathrm{Cu}^{\mathrm{II}}$. 


\section{Native ESI-MS based titrations}

For the titration, samples with different amounts of $\mathrm{Cu}^{\mathrm{II}}(0 \ldots 2$ equiv.; steps of 0.2 equiv) were analyzed by highresolution ESI-TOF mass spectrometry keeping conditions constant. The relative intensities of $\mathrm{Cu}^{\mathrm{II}}$-bound and -unbound G-quadruplex were then plotted against the equiv. of added $\mathrm{Cu}^{\mathrm{II}}$ (Figure S42; normalized plot). For htelL $\mathbf{L}_{3} \mathrm{D}$, the signal corresponding to the $\mathrm{Cu}$-bound form $\mathrm{Cu} @$ htel $^{R_{3} \mathrm{D}}$ showed significantly higher intensities compared to $\mathrm{Cu} @$ htel $\mathbf{L}^{{ }_{2}} \mathrm{~F}$ at same copper equiv. added (with earlier onset of stabilization from 0 to 0.5 equiv.), which can again be explained by stronger $\mathrm{Cu}^{\mathrm{II}}$ binding of htel $\mathbf{L}^{R}{ }_{3} \mathrm{D}$, featuring three ligands $\mathbf{L}^{R}$ as opposed to only two neighbouring $\mathbf{L}^{R}$ donors in sequence htel $\mathbf{L}^{R_{2}} \mathrm{~F}$. Samples containing $25 \mu \mathrm{M}$ DNA, $1 \mathrm{mM} \mathrm{KCl}$ and $100 \mathrm{mM} \mathrm{TMAA} \mathrm{pH}$ 6.8 were annealed by heating them to $85^{\circ} \mathrm{C}$ and cooling them down to $4{ }^{\circ} \mathrm{C}$ with $0.5^{\circ} \mathrm{C} \mathrm{min}^{-1}$. After annealing samples were diluted 1:1 with acetonitrile resulting in a final concentration of $12.5 \mu \mathrm{M}$ DNA, 12.5 or $15 \mu \mathrm{M} \mathrm{CuSO}_{4}, 0.5 \mathrm{mM}$ $\mathrm{KCl}$ and 50 mM TMAA pH 6.8 .

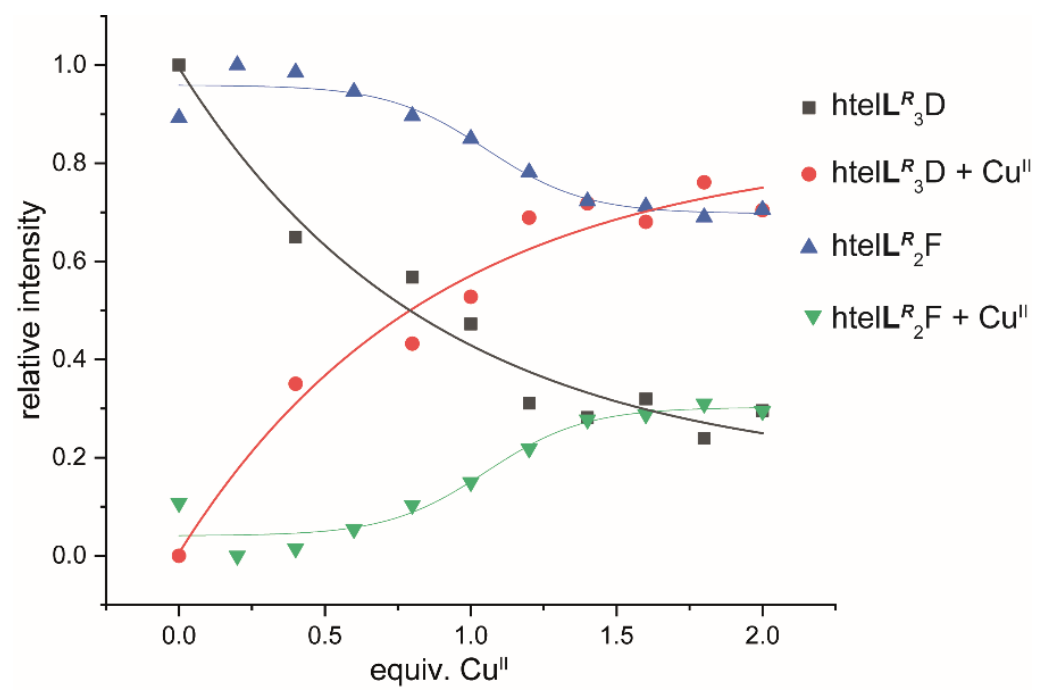

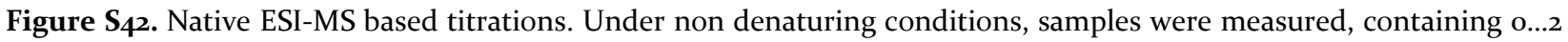
equiv. $\mathrm{Cu}^{\mathrm{II}}$ in 0.2 equiv. steps. The normalized peak intensities of $\mathrm{Cu}^{\mathrm{II}}$-free $\mathrm{G}$-quadruplex and $\mathrm{Cu}^{\mathrm{II}}$-bound $\mathrm{G}$-quadruplex

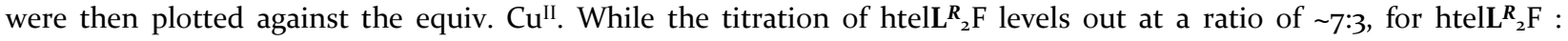

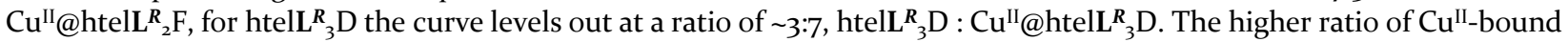
G-quadruplex for htel $L^{R} \mathrm{D}$, featuring three ligands, is explained with a stronger binding of $\mathrm{Cu}^{\mathrm{II}}$ as compred to htel $\mathrm{L}_{2} \mathrm{~F}$, containing only two ligands (in the same loop). Conditions: $12.5 \mu \mathrm{M}$ DNA, 0.5 mM KCl, 50 mM TMAA pH 6.8, $\mathrm{H}_{2} \mathrm{O}: \mathrm{ACN}$ 1:1. 


\section{MD simulations}

\section{Determination of force field parameters}

Simulations were performed using GROMACS 2020 ${ }^{[12-18]}$ The AMBER force field ff99bsc1 was modified with new force field parameters for the imidazole ligandosides based on analogy to existing force field parameters. ${ }^{[19,20]}$ Adjacent ligands were named differently, to avoid ambiguity in the parameterization. Forcefield parameters for the imidazole ligands were based on a previous publication. ${ }^{[1]}$ To calculate the parameters for the Michael-acceptor and the $\mathrm{Cu}^{\mathrm{II}}$ substrate complex, first the complex was geometry optimized with Gaussian on the B3LYP/6-31G(d) level of theory (Fig. 12). ${ }^{[21-25]}$ The geometry optimized complex was used as the input for parmchk2 and MCPB.py from AmberTools 19. ${ }^{[22,26,27]}$ In MCPB.py, the Seminario Method was used. ${ }^{[28]}$ The new forcefield parameters are summarized in Tab. 10-12. Improper dihedrals were used to maintain coplanarity between the imidazole plane and the imidazole - metal plane. The respective parameters for $\mathrm{Cu}^{\mathrm{II}}$ were estimated based on literature values. ${ }^{[25]}$

The crystal structure of a unimolecular antiparallel basket-type (pdb: 143D) G-quadruplex was modified with the Imidazole ligandosides to obtain the starting structure. ${ }^{[29]}$ The modified starting structures were placed in a periodic rhombic bounding box and energy minimized for 2000 steps using steepest descent $\left(600 \mathrm{~kJ} \mathrm{~mol}^{-1} \mathrm{~nm}^{1}\right.$ tolerance). The system was solvated using a TIP3P water model and negative charges on the phosphates were neutralized with $\mathrm{Na}^{+}$or $\mathrm{K}^{+}$and the electrolyte concentration was set to $100 \mathrm{mM}$.

After neutralization, the structure was energy minimized using at first 500 steps of steepest descent with a tolerance of $500 \mathrm{~kJ} \mathrm{~mol}^{-1} \mathrm{~nm}^{-1}$, followed by 3000 steps of conjugate gradient minimization with a tolerance of $300 \mathrm{~kJ} \mathrm{~mol}^{-1} \mathrm{~nm}^{-1}$. A cut-off of the non-bonded Lennard Jones Potential was set to $1.3 \mathrm{~nm}$. Coulombic interactions were treated using a Particle-mesh Ewald summation. Prior to the MD simulation, the starting structure was subjected to three rounds of energy minimization. In a first round using an NVT ensemble (constraints $1000 \mathrm{~kJ} \mathrm{~mol}^{-1} \AA^{-2}$, time step $2 \mathrm{fs}$; Temperature coupling modified Berendsen, $298 \mathrm{~K}$ ) and positional constraints on heavy atoms was simulated for 100 ps, followed by 100 ps with additional pressure coupling (isotropic, Berendsen, 1 bar, time constant for coupling 0.1 ps, compressibility $\left.4.510^{-5}\right)$ and in a last run $100 \mathrm{ps}$ were simulated with lower applied positional constraints (100 kJ $\mathrm{mol}^{-1} \AA^{-2}$, Nose-Hoover temperature coupling 2 ps coupling, Parinello-Rahman isotropic pressure coupling, 2 ps coupling time). Prior to the production run a short MD simulation was run for $200 \mathrm{ps}$ using the same settings as for the production run. The final simulation was simulated for 50/100 ns, writing coordinates after every 10 ps. After the production run, trajectories were centred and aligned. After clustering of the MD trajectory with Chimera, a representative snapshot was taken from one of the most abundant clusters. In the rmsd plots, the first frame was used as the reference. 


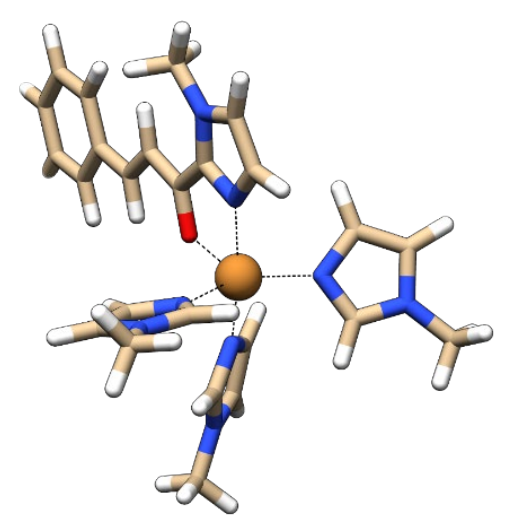

Figure S43. Geometry optimized $\mathrm{Cu}^{\mathrm{II}}$-substrate complex on the B3LYP/6-31G(d) level of theory.
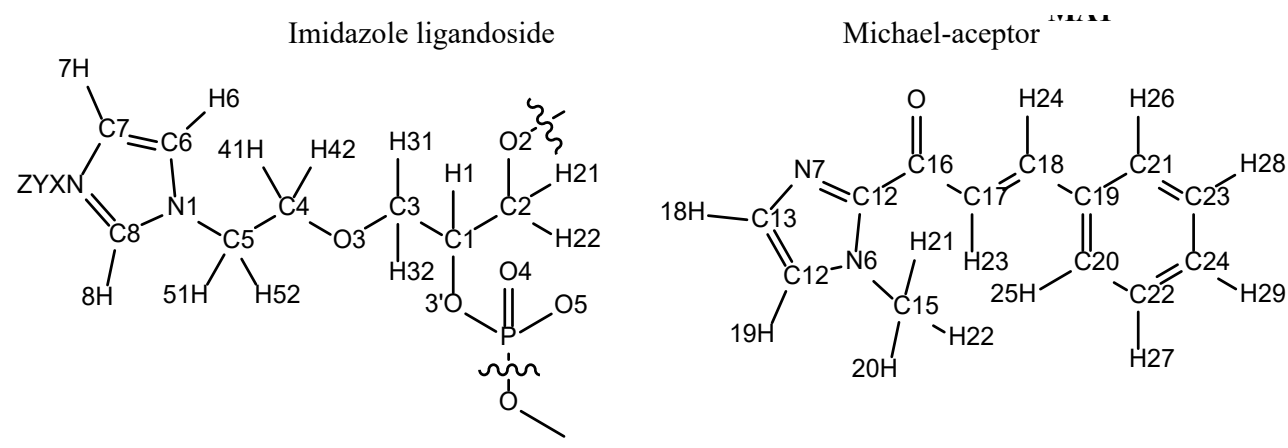

Figure S44. Numbering of the imidazole ligand $\mathbf{L}$ and Michael-acceptor 1.

Table S12: Added bond parameters.

$\begin{array}{lllccl}\mathrm{CT} & \mathrm{NB} & 1 & 0.1475 & 282001.6 & \text { same as CT N* } \\ \mathrm{CE} & \mathrm{CI} & 1 & 0.1526 & 259408 & \text { same as CE CT } \\ \mathrm{CV} & \mathrm{NB} & 1 & 0.1394 & 343088 & \text { same as CV NB } \\ \mathrm{CV} & \mathrm{CW} & 1 & 0.1375 & 428441.6 & \text { same as CC CV } \\ \mathrm{CR} & \mathrm{NX} / \mathrm{Y} / \mathrm{Z} & 1 & 0.1335 & 408358.4 & \text { same as CR NB } \\ \mathrm{CW} & \mathrm{NX} / \mathrm{Y} / \mathrm{Z} & 1 & 0.1394 & 343088 & \text { same as CV NB } \\ \mathrm{CC} & \mathrm{H} 4 & 1 & 0.10817 & 147276.8 & \text { parmchk2 } \\ \mathrm{CC} & \mathrm{CD} & 1 & 0.13729 & 209576.6 & \text { parmchk2 } \\ \mathrm{CD} & \mathrm{H} 4 & 1 & 0.10817 & 147276.8 & \text { parmchk2 } \\ \mathrm{C} 3 & \mathrm{NA} & 1 & 0.14629 & 137109.7 & \text { parmchk2 } \\ \mathrm{C} 3 & \mathrm{H} 1 & 1 & 0.10969 & 138323 & \text { parmchk2 } \\ \mathrm{CC} & \mathrm{CF} & 1 & 0.13656 & 214639.2 & \text { parmchk2 } \\ \mathrm{CF} & \mathrm{CF} & 1 & 0.14574 & 160163.5 & \text { parmchk2 } \\ \mathrm{CJ} & \mathrm{CF} & 1 & 0.13509 & 223250.2 & \text { parmchk2 } \\ \mathrm{CJ} & \mathrm{HA} & 1 & 0.10883 & 143302 & \text { parmchk2 } \\ \mathrm{CA} & \mathrm{CJ} & 1 & 0.14763 & 151167.9 & \text { parmchk2 } \\ \mathrm{CF} & \mathrm{OS} & 1 & 0.1371 & 156858.2 & \text { parmchk2 } \\ \mathrm{CF} & \mathrm{HA} & 1 & 0.10883 & 143302 & \text { parmchk2 } \\ \mathrm{CD} & \mathrm{NA} & 1 & 0.13802 & 178154.7 & \text { parmchk2 } \\ \mathrm{CU} & \mathrm{NX} & 1 & 0.20314 & 22342.6 & \text { MCPB.py } \\ \mathrm{CU} & \mathrm{NY} & 0.19842 & 32760.7 & \text { MCPB.py }\end{array}$




$\begin{array}{lllccl}\text { CU } & \text { NZ } & 1 & 0.20597 & 18911.7 & \text { MCPB.py } \\ \text { CU } & \text { NA } & 1 & 0.20087 & 26275.5 & \text { MCPB.py } \\ \text { CU } & \text { OS } & 1 & 0.22003 & 3138 & \text { MCPB.py }\end{array}$

Table S13: Added angle parameters.

\begin{tabular}{|c|c|c|c|c|c|c|}
\hline OS & $\mathrm{CE}$ & CI & 1 & 109.5 & 418.4 & same as OS CE CT \\
\hline OS & $\mathrm{CI}$ & $\mathrm{CE}$ & 1 & 109.5 & 418.4 & same as OS CE CT \\
\hline $\mathrm{CE}$ & $\mathrm{CI}$ & $\mathrm{H} 1$ & 1 & 109.5 & 418.4 & same as CE CT H1 \\
\hline $\mathrm{CI}$ & $\mathrm{CE}$ & $\mathrm{H} 1$ & 1 & 109.5 & 418.4 & same as CE CT H1 \\
\hline $\mathrm{CT}$ & $\mathrm{CE}$ & $\mathrm{CI}$ & 1 & 109.5 & 334.7 & same as CT CE CT \\
\hline $\mathrm{CT}$ & $\mathrm{CT}$ & NB & 1 & 109.5 & 418.4 & same as CT CT N* \\
\hline $\mathrm{CE}$ & $\mathrm{CI}$ & $\mathrm{OH}$ & 1 & 109.5 & 418.4 & same as $\mathrm{CT} \mathrm{CI} \mathrm{OH}$ \\
\hline H1 & $\mathrm{CT}$ & NB & 1 & 109.5 & 418.4 & same as $\mathrm{H} 1 \mathrm{CT} \mathrm{N}^{*}$ \\
\hline $\mathrm{CW}$ & $\mathrm{CV}$ & $\mathrm{H} 4$ & 1 & 120.0 & 418.4 & same as $\mathrm{CC} \mathrm{CV} \mathrm{H4}$ \\
\hline $\mathrm{CV}$ & $\mathrm{CW}$ & $\mathrm{H} 4$ & 1 & 120.0 & 418.4 & same as $\mathrm{CC} \mathrm{CV} \mathrm{H4}$ \\
\hline $\mathrm{CR}$ & NB & $\mathrm{CT}$ & 1 & 128.8 & 585.8 & same as $\mathrm{CK} \mathrm{N}^{*} \mathrm{CT}$ \\
\hline $\mathrm{CV}$ & NB & $\mathrm{CT}$ & 1 & 128.8 & 585.8 & same as $\mathrm{CK}^{*} \mathrm{CT}$ \\
\hline $\mathrm{CW}$ & $\mathrm{CV}$ & NB & 1 & 120.0 & 585.8 & same as CW CC NB \\
\hline CR & NX/NY/NZ & $\mathrm{CW}$ & 1 & 120.0 & 585.8 & same as CR NA CW \\
\hline NB & $\mathrm{CR}$ & NX/NY/NZ & 1 & 120.0 & 585.8 & same as NA CR NA \\
\hline NX/NY/NZ & $\mathrm{CW}$ & $\mathrm{CV}$ & 1 & 120.0 & 585.8 & same as CC CW NA \\
\hline $\mathrm{H} 4$ & $\mathrm{CW}$ & $\mathrm{NX} / \mathrm{NY} / \mathrm{NZ}$ & 1 & 120.0 & 418.4 & same as $\mathrm{H} 4 \mathrm{CW}$ NA \\
\hline H5 & $\mathrm{CR}$ & NX/NY/NZ & 1 & 120.0 & 418.4 & same as H5 CR NA \\
\hline $\mathrm{CU}$ & NX & $\mathrm{CW}$ & 1 & 127.1 & 318.6 & MCPB.py \\
\hline $\mathrm{CU}$ & NY & $\mathrm{CW}$ & 1 & 126.8 & 374.0 & MCPB.py \\
\hline $\mathrm{CU}$ & NZ & $\mathrm{CW}$ & 1 & 126.9 & 265.6 & MCPB.py \\
\hline $\mathrm{CU}$ & NA & $\mathrm{CC}$ & 1 & 126.1 & 301.0 & MCPB.py \\
\hline $\mathrm{CU}$ & OS & $\mathrm{CF}$ & 1 & 115.3 & 149.5 & MCPB.py \\
\hline $\mathrm{CU}$ & NX & $\mathrm{CR}$ & 1 & 127.1 & 318.6 & MCРB.py \\
\hline $\mathrm{CU}$ & NY & $\mathrm{CR}$ & 1 & 126.8 & 372.0 & MCPB.py \\
\hline $\mathrm{CU}$ & $\mathrm{NZ}$ & $\mathrm{CR}$ & 1 & 126.9 & 265.6 & МСРВ.py \\
\hline $\mathrm{CU}$ & NA & $\mathrm{CC}$ & 1 & 126.1 & 301.0 & MCPB.py \\
\hline NY & $\mathrm{CU}$ & NX & 1 & 92.5 & 244.8 & MCPB.py \\
\hline $\mathrm{NZ}$ & $\mathrm{CU}$ & $\mathrm{NX}$ & 1 & 135.6 & 100.2 & МСРВ.py \\
\hline $\mathrm{NZ}$ & $\mathrm{CU}$ & NY & 1 & 93.2 & 229.2 & МСРВ.py \\
\hline NA & $\mathrm{CU}$ & $\mathrm{NX}$ & 1 & 93.2 & 209.6 & МCPB.py \\
\hline NA & $\mathrm{CU}$ & NY & 1 & 165.3 & 281.6 & MCРB.py \\
\hline NA & $\mathrm{CU}$ & NZ & 1 & 92.1 & 175.5 & МСРВ.py \\
\hline OS & $\mathrm{CU}$ & $\mathrm{NX}$ & 1 & 118.3 & 58.5 & MCPB.py \\
\hline OS & $\mathrm{CU}$ & NY & 1 & 88.6 & 174.3 & МСРВ.py \\
\hline OS & $\mathrm{CU}$ & NZ & 1 & 105.9 & 70.1 & MCPB.py \\
\hline OS & $\mathrm{CU}$ & NA & 1 & 76.8 & 190.0 & MCPB.py \\
\hline $\mathrm{H} 4$ & $\mathrm{CC}$ & NA & 1 & 120.5 & 208.4 & parmchk2 \\
\hline $\mathrm{CD}$ & $\mathrm{CC}$ & $\mathrm{H} 4$ & 1 & 128.5 & 197.9 & parmchk2 \\
\hline $\mathrm{CD}$ & $\mathrm{CC}$ & NA & 1 & 107.0 & 307.1 & parmchk2 \\
\hline $\mathrm{CC}$ & $\mathrm{CD}$ & $\mathrm{H} 4$ & 1 & 128.5 & 197.9 & parmchk2 \\
\hline $\mathrm{H} 4$ & $\mathrm{CD}$ & NA & 1 & 120.5 & 208.4 & parmchk2 \\
\hline $\mathrm{C} 3$ & NA & $\mathrm{CD}$ & 1 & 126.5 & 259.0 & parmchk2 \\
\hline
\end{tabular}




$\begin{array}{lllllll}\text { CC } & \text { NA } & \text { CD } & 1 & 128.0 & 265.7 & \text { parmchk2 } \\ \text { H1 } & \text { C3 } & \text { NA } & 1 & 108.8 & 208.4 & \text { parmchk2 } \\ \text { NA } & \text { CC } & \text { NA } & 1 & 106.6 & 314.6 & \text { parmchk2 } \\ \text { CC } & \text { NA } & \text { CC } & 1 & 109.9 & 286.6 & \text { parmchk2 } \\ \text { CF } & \text { CC } & \text { NA } & 1 & 124.9 & 284.9 & \text { parmchk2 } \\ \text { CC } & \text { CF } & \text { OS } & 1 & 115.9 & 286.2 & \text { parmchk2 } \\ \text { CC } & \text { CF } & \text { CF } & 1 & 124.4 & 270.7 & \text { parmchk2 } \\ \text { CF } & \text { CF } & \text { OS } & 1 & 115.2 & 289.5 & \text { parmchk2 } \\ \text { CJ } & \text { CF } & \text { CF } & 1 & 124.2 & 272.0 & \text { parmchk2 } \\ \text { CF } & \text { CF } & \text { HA } & 1 & 116.7 & 197.5 & \text { parmchk2 } \\ \text { CF } & \text { CJ } & \text { HA } & 1 & 118.2 & 208.4 & \text { parmchk2 } \\ \text { CA } & \text { CJ } & \text { CF } & 1 & 127.5 & 266.5 & \text { parmchk2 } \\ \text { CA } & \text { CJ } & \text { HA } & 1 & 115.1 & 196.6 & \text { parmchk2 } \\ \text { CA } & \text { CA } & \text { CJ } & 1 & 120.8 & 269.9 & \text { parmchk2 } \\ \text { C3 } & \text { NA } & \text { CC } & 1 & 126.5 & 259.0 & \text { parmchk2 } \\ \text { H1 } & \text { C3 } & \text { H1 } & 1 & 108.5 & 164.0 & \text { parmchk2 } \\ \text { CJ } & \text { CF } & \text { HA } & 1 & 118.2 & 208.4 & \text { parmchk2 } \\ \text { CC } & \text { CD } & \text { NA } & 1 & 107.0 & 307.1 & \text { parmchk2 }\end{array}$

Table S14: Added dihedral parameters.

\begin{tabular}{|c|c|c|c|c|c|c|c|}
\hline $\mathrm{CC}$ & $\mathrm{H} 4$ & $\mathrm{CD}$ & NA & 4 & 180.0 & 4.6 & parmchk2 \\
\hline $\mathrm{CD}$ & $\mathrm{H} 4$ & $\mathrm{CC}$ & NA & 4 & 180.0 & 4.6 & parmchk2 \\
\hline $\mathrm{C} 3$ & $\mathrm{CC}$ & NA & $\mathrm{CC}$ & 4 & 180.0 & 4.6 & parmchk2 \\
\hline $\mathrm{C} 3$ & $\mathrm{CD}$ & NA & $\mathrm{CD}$ & 4 & 180.0 & 4.6 & parmchk2 \\
\hline $\mathrm{C} 3$ & $\mathrm{CC}$ & NA & $\mathrm{CD}$ & 4 & 180.0 & 4.6 & parmchk2 \\
\hline $\mathrm{CF}$ & NA & $\mathrm{CC}$ & NA & 4 & 180.0 & 4.6 & parmchk2 \\
\hline $\mathrm{CC}$ & $\mathrm{CF}$ & $\mathrm{CF}$ & OS & 4 & 180.0 & 4.6 & parmchk2 \\
\hline CJ & $\mathrm{CF}$ & $\mathrm{CF}$ & HA & 4 & 180.0 & 4.6 & parmchk2 \\
\hline $\mathrm{CA}$ & $\mathrm{CF}$ & CJ & HA & 4 & 180.0 & 4.6 & parmchk2 \\
\hline CA & CA & CA & CJ & 4 & 180.0 & 4.6 & parmchk2 \\
\hline CA & CA & $\mathrm{CA}$ & HA & 4 & 180.0 & 4.6 & parmchk2 \\
\hline $\mathrm{CW}$ & $\mathrm{CR}$ & NX & $\mathrm{CU}$ & 4 & 180.0 & 30.0 & estimated based on literature \\
\hline $\mathrm{CW}$ & $\mathrm{CR}$ & NY & $\mathrm{CU}$ & 4 & 180.0 & 30.0 & estimated based on literature \\
\hline $\mathrm{CW}$ & $\mathrm{CR}$ & NX & $\mathrm{CU}$ & 4 & 180.0 & 30.0 & estimated based on literature \\
\hline $\mathrm{CW}$ & $\mathrm{CR}$ & NY & $\mathrm{CU}$ & 4 & 180.0 & 30.0 & estimated based on literature \\
\hline $\mathrm{CW}$ & CR & $\mathrm{NZ}$ & $\mathrm{CU}$ & 4 & 180.0 & 30.0 & estimated based on literature \\
\hline $\mathrm{CC}$ & $\mathrm{CC}$ & NA & $\mathrm{CU}$ & 4 & 180.0 & 30.0 & estimated based on literature \\
\hline $\mathrm{X}$ & $\mathrm{CE}$ & $\mathrm{CI}$ & $\mathrm{X}$ & 9 & 0.0 & 0.7 & same as X CT CT X \\
\hline CT & $\mathrm{CE}$ & CI & $\mathrm{OH}$ & 9 & 191.0 & 4.9 & same as $\mathrm{CE} \mathrm{CT} \mathrm{CI} \mathrm{OH}$ \\
\hline $\mathrm{P}$ & OS & CI & $\mathrm{CE}$ & 9 & 297.8 & 4.5 & same as P OS CE CT \\
\hline $\mathrm{P}$ & OS & $\mathrm{CI}$ & $\mathrm{CE}$ & 9 & 200.6 & 1.3 & same as P OS CE CT \\
\hline $\mathrm{P}$ & OS & $\mathrm{CI}$ & $\mathrm{CE}$ & 9 & 151.6 & 0.2 & same as P OS CE CT \\
\hline $\mathrm{X}$ & $\mathrm{CV}$ & $\mathrm{CW}$ & $\mathrm{X}$ & 9 & 180.0 & 2.2 & same as X CC CW X \\
\hline $\mathrm{X}$ & CT & NB & $\mathrm{X}$ & 9 & 0.0 & 0.0 & same as $\mathrm{X} \mathrm{CT} \mathrm{N*X}$ \\
\hline $\mathrm{X}$ & $\mathrm{CW}$ & $\mathrm{NX} / \mathrm{Y} / \mathrm{Z}$ & $\mathrm{X}$ & 9 & 180.0 & 6.3 & same as X CW NA X \\
\hline $\mathrm{X}$ & $\mathrm{NX} / \mathrm{Y} / \mathrm{Z}$ & $\mathrm{CR}$ & $\mathrm{X}$ & 9 & 180.0 & 9.7 & same as X CR NA X \\
\hline $\mathrm{X}$ & $\mathrm{CW}$ & NA & $\mathrm{X}$ & 9 & 180.0 & 6.3 & same as X CW NA X \\
\hline $\mathrm{X}$ & NA & $\mathrm{CR}$ & $\mathrm{X}$ & 9 & 180.0 & 9.7 & same as X CR NA X \\
\hline
\end{tabular}




$\begin{array}{llllllll}\mathrm{CT} & \mathrm{CE} & \mathrm{CI} & \mathrm{OS} & 9 & 191.0 & 4.9 & \text { same as OS CI CT CT } \\ \mathrm{CT} & \mathrm{CE} & \mathrm{CI} & \mathrm{OS} & 9 & 295.6 & 0.4 & \text { same as OS CI CT CT } \\ \mathrm{CT} & \mathrm{CE} & \mathrm{CI} & \mathrm{OS} & 9 & 348.1 & 4.0 & \text { same as OS CI CT CT } \\ \mathrm{CC} & \mathrm{CF} & \mathrm{OS} & \mathrm{C} 3 & 9 & 180.0 & 8.8 & \text { parmchk2 } \\ \mathrm{CF} & \mathrm{CF} & \mathrm{OS} & \mathrm{C} 3 & 9 & 180.0 & 8.8 & \text { parmchk2 } \\ \mathrm{NA} & \mathrm{CC} & \mathrm{CF} & \mathrm{OS} & 9 & 180.0 & 66.9 & \text { parmchk2 } \\ \mathrm{NA} & \mathrm{CC} & \mathrm{CF} & \mathrm{CF} & 9 & 180.0 & 66.9 & \text { parmchk2 } \\ \mathrm{CA} & \mathrm{CA} & \mathrm{CJ} & \mathrm{CF} & 9 & 180.0 & 11.7 & \text { parmchk2 } \\ \mathrm{CA} & \mathrm{CA} & \mathrm{CJ} & \mathrm{HA} & 9 & 180.0 & 11.7 & \text { parmchk2 } \\ \mathrm{X} & \mathrm{CF} & \mathrm{CF} & \mathrm{X} & 9 & 180.0 & 16.7 & \text { parmchk2 } \\ \mathrm{X} & \mathrm{C} 3 & \mathrm{NA} & \mathrm{X} & 9 & 0.0 & 0.0 & \text { parmchk2 } \\ \mathrm{X} & \mathrm{CD} & \mathrm{NA} & \mathrm{X} & 9 & 180.0 & 28.5 & \text { parmchk2 } \\ \mathrm{X} & \mathrm{CC} & \mathrm{CD} & \mathrm{X} & 9 & 180.0 & 66.9 & \text { parmchk2 } \\ \mathrm{X} & \mathrm{CJ} & \mathrm{CF} & \mathrm{X} & 9 & 180.0 & 111.3 & \text { parmchk2 }\end{array}$

\section{Calculation of RESP charges}

Partial point charges were derived by RESP charge fitting. Therefore the imidazole ligands and the $\mathrm{Cu}^{\mathrm{II}}$ complex were fragmented according to the original capping scheme, ${ }^{[30]}$ structure optimized (B3LYP/6-311+G(d,p)) and submitted to the REDServer-Development (Table S15). ${ }^{[1,31-34]}$ Inter-molecular and intra-molecular charge constraints were used to maintain the correct total charge of the ligand. The Charge of $\mathrm{Cu}^{\mathrm{II}}$ was constrained to 0.5.

Ligand fragment
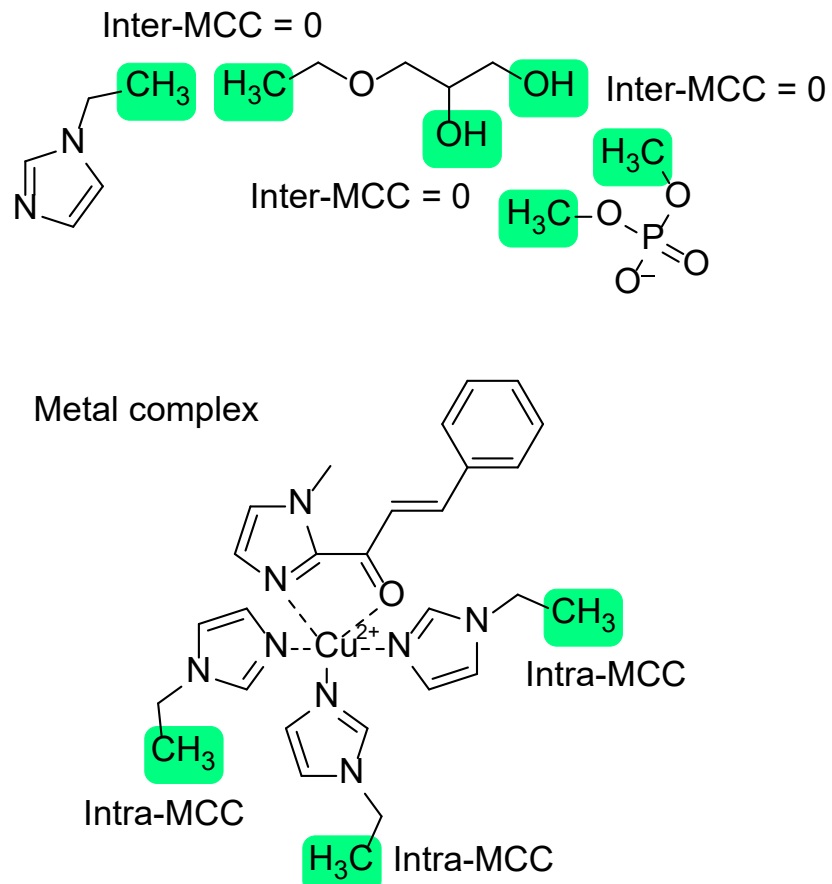

Figure S45. Capping scheme used for the RESP-charge fitting. 
Table S15: Atom types and RESP charges of the imidazole ligands $\mathbf{L}$ and Michael acceptor $\mathbf{1}$.

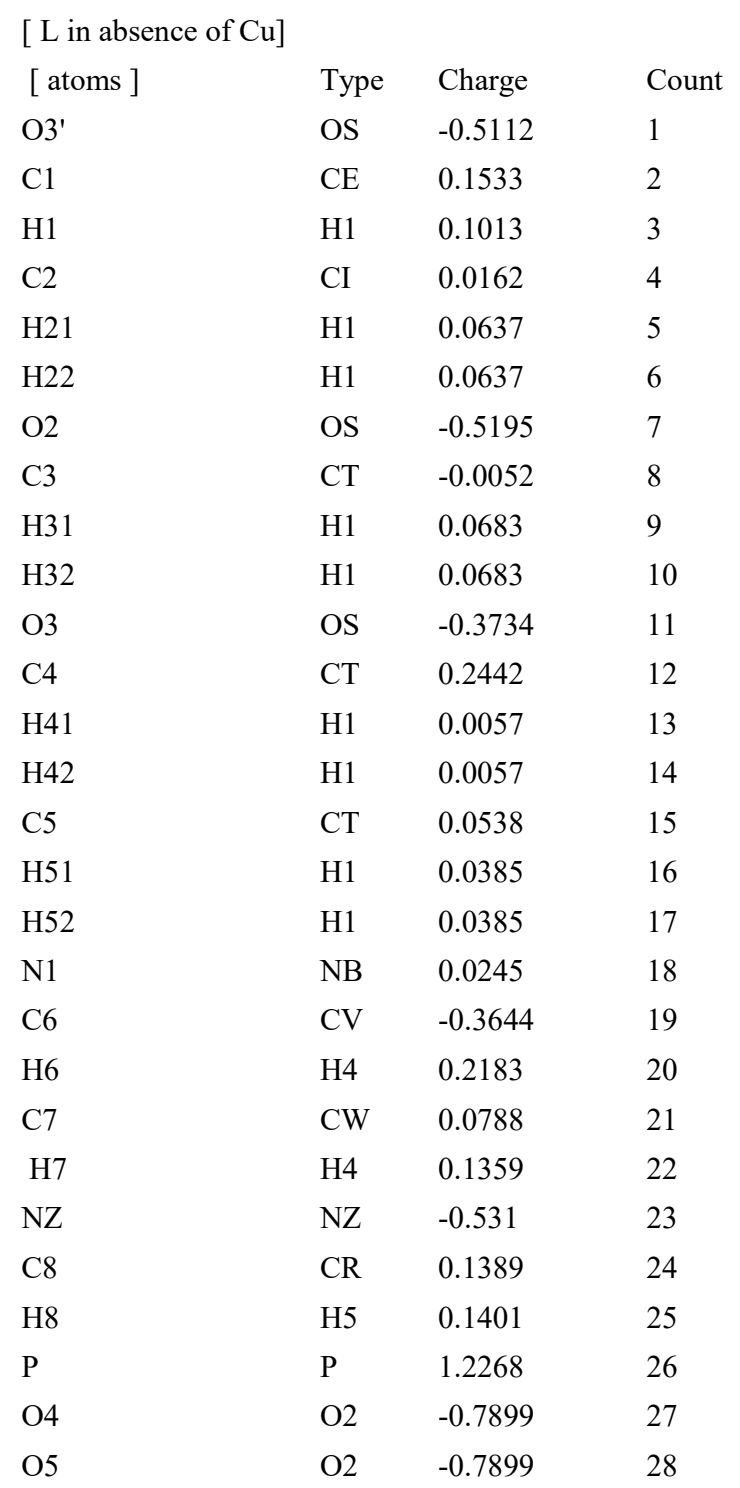

\section{[ LXI ]}

[ atoms ]

O3'

C1

$\mathrm{H} 1$

$\mathrm{C} 2$

$\mathrm{H} 21$

$\mathrm{H} 22$

$\mathrm{O} 2$

C3

H31

H32

O3

C4

H41

$\begin{array}{lll}\text { Type } & \text { Charge } & \text { Count } \\ \text { OS } & -0.5112 & 1 \\ \text { CE } & 0.1533 & 2 \\ \text { H1 } & 0.1013 & 3 \\ \text { CI } & 0.0162 & 4 \\ \text { H1 } & 0.0637 & 5 \\ \text { H1 } & 0.0637 & 6 \\ \text { OS } & -0.5195 & 7 \\ \text { CT } & -0.0052 & 8 \\ \text { H1 } & 0.0683 & 9 \\ \text { H1 } & 0.0683 & 10 \\ \text { OS } & -0.3734 & 11 \\ \text { CT } & 0.2442 & 12 \\ \text { H1 } & 0.0057 & 13\end{array}$

[ LYI ]

$\begin{array}{llll}\text { [atoms ] } & \text { Type } & \text { Charge } & \text { Count } \\ \text { O3' } & \text { OS } & -0.5112 & 1 \\ \text { C1 } & \text { CE } & 0.1533 & 2 \\ \text { H1 } & \text { H1 } & 0.1013 & 3 \\ \text { C2 } & \text { CI } & 0.0162 & 4 \\ \text { H21 } & \text { H1 } & 0.0637 & 5 \\ \text { H22 } & \text { H1 } & 0.0637 & 6 \\ \text { O2 } & \text { OS } & -0.5195 & 7 \\ \text { C3 } & \text { CT } & -0.0052 & 8 \\ \text { H31 } & \text { H1 } & 0.0683 & 9 \\ \text { H32 } & \text { H1 } & 0.0683 & 10 \\ \text { O3 } & \text { OS } & -0.3734 & 11 \\ \text { C4 } & \text { CT } & 0.2442 & 12 \\ \text { H41 } & \text { H1 } & 0.0057 & 13\end{array}$




$\begin{array}{llllllll}\mathrm{H} 42 & \mathrm{H} 1 & 0.0057 & 14 & \mathrm{H} 42 & \mathrm{H} 1 & 0.0057 & 14 \\ \mathrm{C} 5 & \mathrm{CT} & 0.1372 & 15 & \mathrm{C} 5 & \mathrm{CT} & 0.0648 & 15 \\ \mathrm{H} 51 & \mathrm{H} 1 & 0.0493 & 16 & \mathrm{H} 51 & \mathrm{H} 1 & 0.0744 & 16 \\ \mathrm{H} 52 & \mathrm{H} 1 & 0.0493 & 17 & \mathrm{H} 52 & \mathrm{H} 1 & 0.0744 & 17 \\ \mathrm{~N} 1 & \mathrm{NB} & 0.0207 & 18 & \mathrm{~N} 1 & \mathrm{NB} & 0.0467 & 18 \\ \mathrm{C} 6 & \mathrm{CV} & -0.2102 & 19 & \mathrm{C} 6 & \mathrm{CV} & -0.212 & 19 \\ \mathrm{H} 6 & \mathrm{H} 4 & 0.2368 & 20 & \mathrm{H} 6 & \mathrm{H} 4 & 0.2158 & 20 \\ \mathrm{C} 7 & \mathrm{CW} & -0.1525 & 21 & \mathrm{C} 7 & \mathrm{CW} & -0.1453 & 21 \\ \mathrm{H} 7 & \mathrm{H} 4 & 0.2041 & 22 & \mathrm{H} 7 & \mathrm{H} 4 & 0.2103 & 22 \\ \mathrm{NX} & \mathrm{NX} & -0.1956 & 23 & \mathrm{NY} & \mathrm{NY} & -0.1893 & 23 \\ \mathrm{C} 8 & \mathrm{CR} & -0.0578 & 24 & \mathrm{C} 8 & \mathrm{CR} & -0.0077 & 24 \\ \mathrm{H} 8 & \mathrm{H} 5 & 0.2029 & 25 & \mathrm{H} 8 & \mathrm{H} 5 & 0.1924 & 25 \\ \mathrm{P} & \mathrm{P} & 1.2268 & 26 & \mathrm{P} & \mathrm{P} & 1.2268 & 26 \\ \mathrm{O} 4 & \mathrm{O} 2 & -0.7899 & 27 & \mathrm{O} 4 & \mathrm{O} 2 & -0.7899 & 27 \\ \mathrm{O} 5 & \mathrm{O} 2 & -0.7899 & 28 & \mathrm{O} 5 & \mathrm{O} 2 & -0.7899 & 28\end{array}$

[ LZI ]

\begin{tabular}{llll} 
[atoms ] & Type & Charge & Count \\
O3' & OS & -0.5112 & 1 \\
C1 & CE & 0.1533 & 2 \\
H1 & H1 & 0.1013 & 3 \\
C2 & CI & 0.0162 & 4 \\
H21 & H1 & 0.0637 & 5 \\
H22 & H1 & 0.0637 & 6 \\
O2 & OS & -0.5195 & 7 \\
C3 & CT & -0.0052 & 8 \\
H31 & H1 & 0.0683 & 9 \\
H32 & H1 & 0.0683 & 10 \\
O3 & OS & -0.3734 & 11 \\
C4 & CT & 0.2442 & 12 \\
H41 & H1 & 0.0057 & 13 \\
H42 & H1 & 0.0057 & 14 \\
C5 & CT & 0.068 & 15 \\
H51 & H1 & 0.0768 & 16 \\
H52 & H1 & 0.0768 & 17 \\
N1 & NB & 0.051 & 18 \\
C6 & CV & -0.2222 & 19 \\
H6 & H4 & 0.2199 & 20 \\
C7 & CW & -0.1394 & 21 \\
H7 & H4 & 0.1864 & 22 \\
NZ & NZ & -0.2066 & 23 \\
C8 & CR & 0.0073 & 24 \\
H8 & H5 & 0.1684 & 25 \\
P & P & 1.2268 & 26 \\
O4 & O2 & -0.7899 & 27 \\
O5 & O2 & -0.7899 & 28 \\
& & & \\
\hline & &
\end{tabular}

[ Sub ]

$\begin{array}{llll}\text { [atoms ] } & \text { Type } & \text { Charge } & \text { Count } \\ \text { C12 } & \text { CC } & 0.1542 & 1 \\ \text { C13 } & \text { CC } & -0.2378 & 2 \\ \text { C14 } & \text { CD } & -0.1265 & 3 \\ \text { H18 } & \text { H4 } & 0.2183 & 4 \\ \text { H19 } & \text { H4 } & 0.2356 & 5 \\ \text { N6 } & \text { NA } & -0.032 & 6 \\ \text { N7 } & \text { NA } & -0.09 & 7 \\ \text { C15 } & \text { C3 } & -0.0997 & 8 \\ \text { H20 } & \text { H1 } & 0.1098 & 9 \\ \text { H21 } & \text { H1 } & 0.1098 & 10 \\ \text { H22 } & \text { H1 } & 0.1098 & 11 \\ \text { C16 } & \text { CF } & 0.4263 & 12 \\ \text { O } & \text { OS } & -0.4414 & 13 \\ \text { C17 } & \text { CF } & -0.2613 & 14 \\ \text { H23 } & \text { HA } & 0.1316 & 15 \\ \text { C18 } & \text { CJ } & -0.0447 & 16 \\ \text { H24 } & \text { HA } & 0.1407 & 17 \\ \text { C19 } & \text { CA } & 0.0737 & 18 \\ \text { C20 } & \text { CA } & -0.0993 & 19 \\ \text { C21 } & \text { CA } & -0.0993 & 20 \\ \text { C22 } & \text { CA } & -0.1545 & 21 \\ \text { H25 } & \text { HA } & 0.1261 & 22 \\ \text { C23 } & \text { CA } & -0.1545 & 23 \\ \text { H26 } & \text { HA } & 0.1261 & 24 \\ \text { C24 } & \text { CA } & -0.0492 & 25 \\ \text { H27 } & \text { HA } & 0.1532 & 26 \\ \text { H28 } & \text { HA } & 0.1532 & 27 \\ \text { H29 } & \text { HA } & 0.1424 & 28\end{array}$




\section{Simulations}

Simulations were performed using GROMACS 2019.2. ${ }^{[28-34]}$ As starting structure, the NMR solution structure of an unimolecular antiparallel (143D) G-quadruplex was used and modified. ${ }^{[35]}$ Obtained starting structures were solvated in a rhombic bounding box with TIP3P water. Negative charges on the phosphates were neutralized with $\mathrm{K}^{+}$cations and the concentration of $\mathrm{KCl}$ was set to $100 \mathrm{mM}$. Prior to the MD simulation, the starting structure was subjected to three rounds of energy minimization and equilibrated at $298 \mathrm{~K}$ and 1 bar. The final MD was simulated for $100 \mathrm{~ns}$ in an NPT ensemble. For details see previous publication. ${ }^{[4]}$
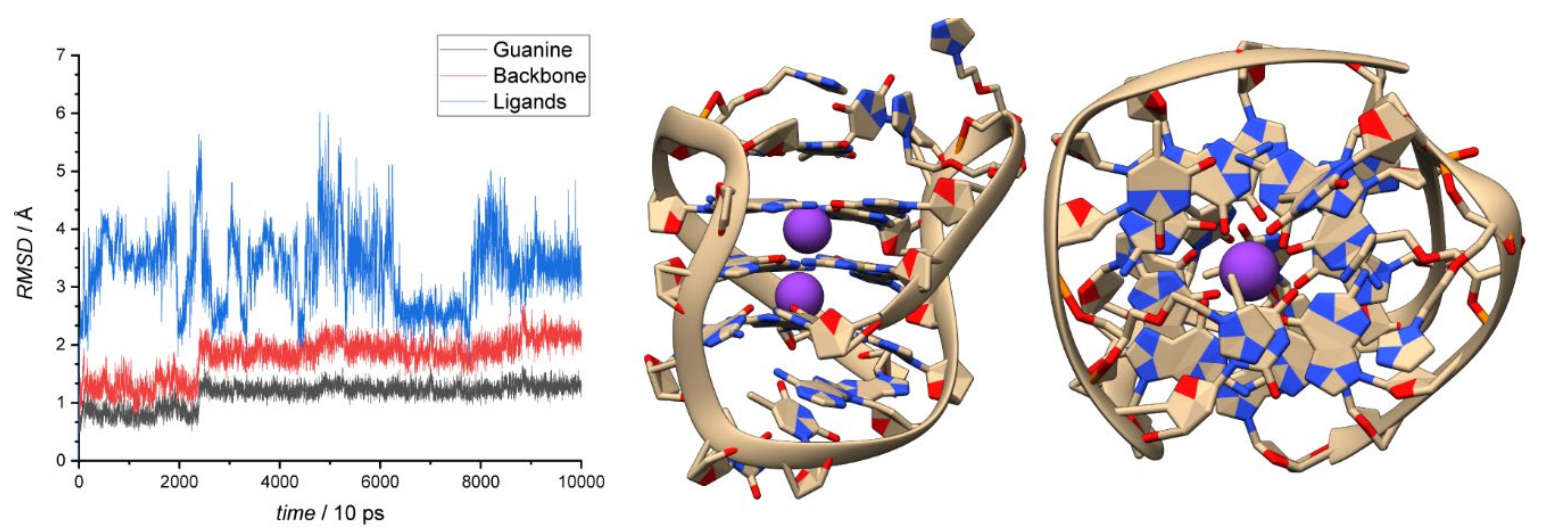

Figure S46. MD simulations of htel $\mathbf{L}^{R_{3} \mathrm{D}}$ in absence of $\mathrm{Cu}^{\mathrm{II}}$.
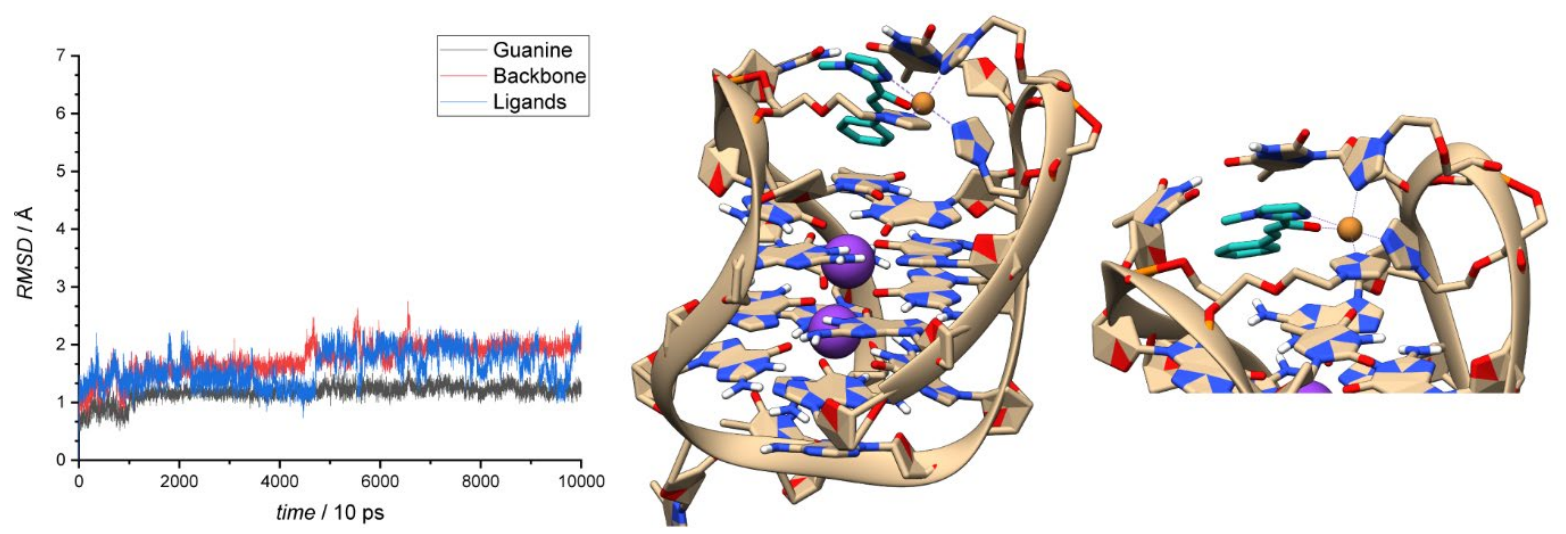

Figure S47. MD simulations of htell ${ }^{R_{3}} \mathrm{D}$ in presence of $\mathrm{Cu}^{\mathrm{II}}$ and the Michael-acceptor.

\section{Analytical ESI-MS}

Oligonucleotide samples were prepared with DNA concentrations of 150 - $300 \mu \mathrm{M}$ in 15 mM TMAA pH 6.8 or TEAA pH 7. ESI mass spectrometry was then performed on a Bruker ESI-timsTOF mass spectrometer (negative mode). For 
calibration of the TOF device, Agilent ESI-Low Concentration Tuning Mix was used. Automatic injection of the samples was achieved with the autosampler of an Agilent Technologies 1260 Infinity system (flow rate: $0.3 \mathrm{~mL} / \mathrm{min}$, solvent: $\mathrm{MeCN} / \mathrm{H}_{2} \mathrm{O}$ 1:1). The ESI mass spectra are shown below.

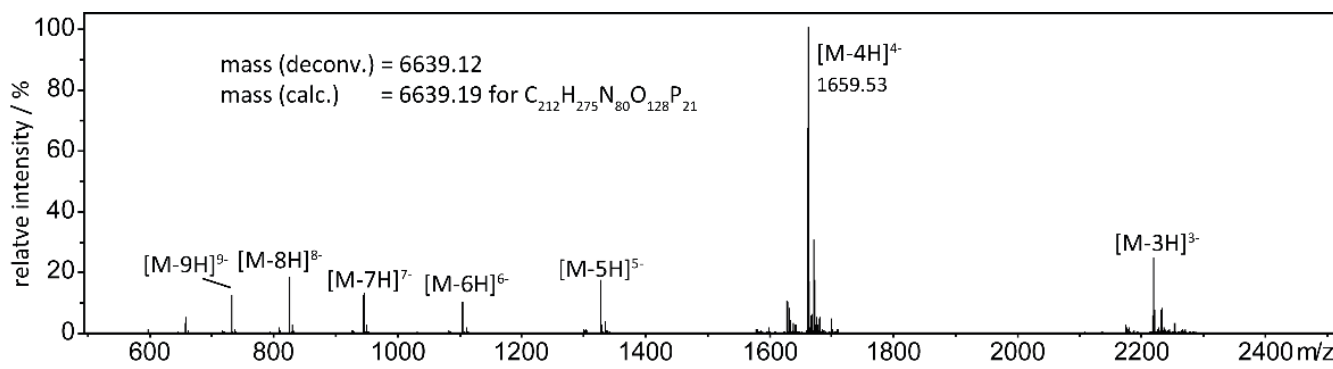

\section{htelL ${ }_{4} \mathrm{~A}$}

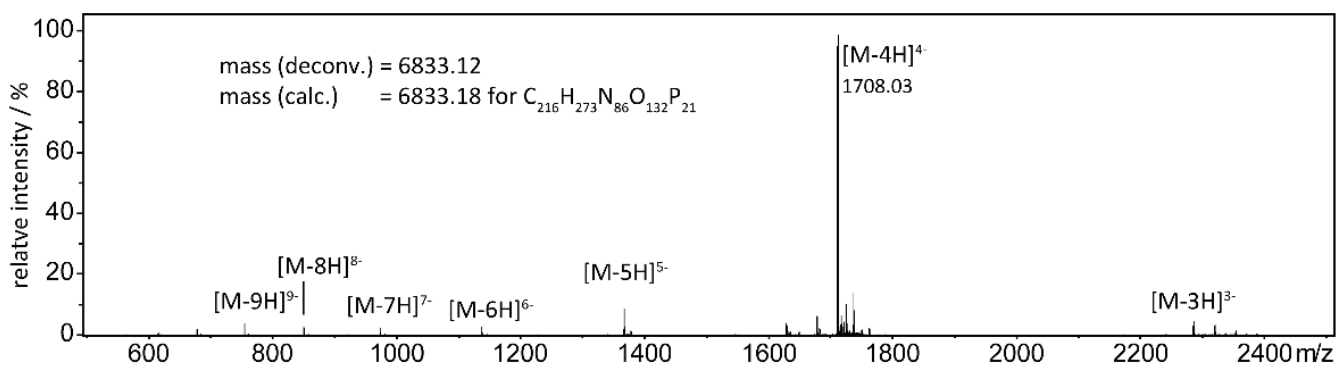

htelL ${ }_{2} \mathrm{~B}$

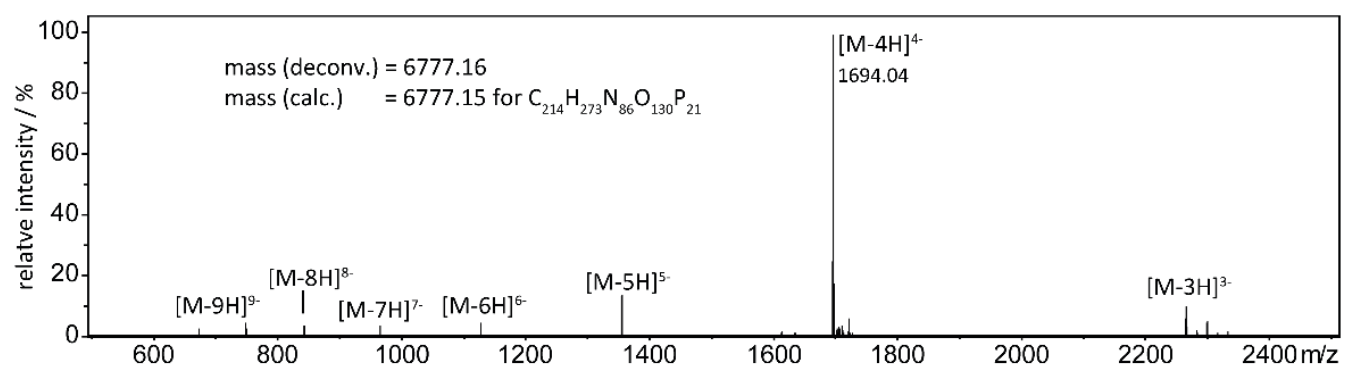

htelL ${ }_{3}^{s} \mathrm{~B}$

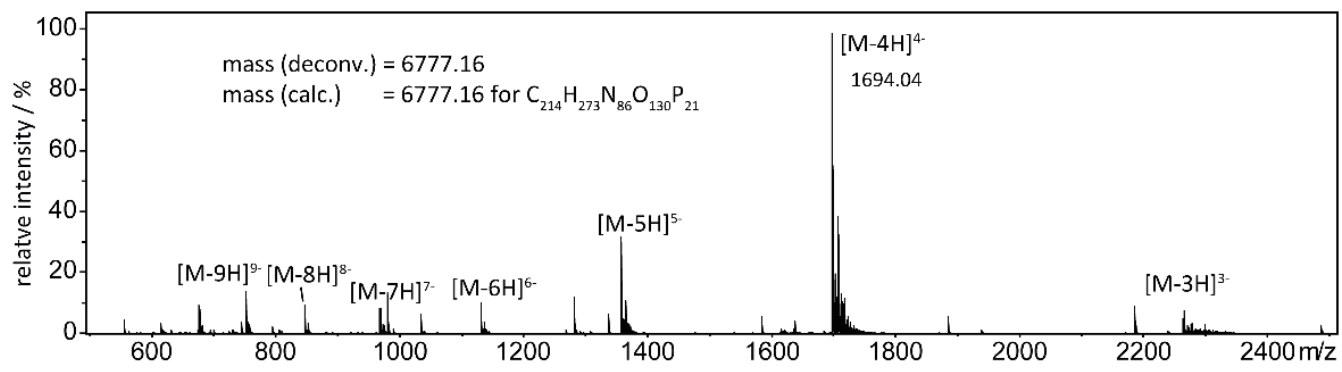

\section{$91 \mathrm{~L}^{R}$}

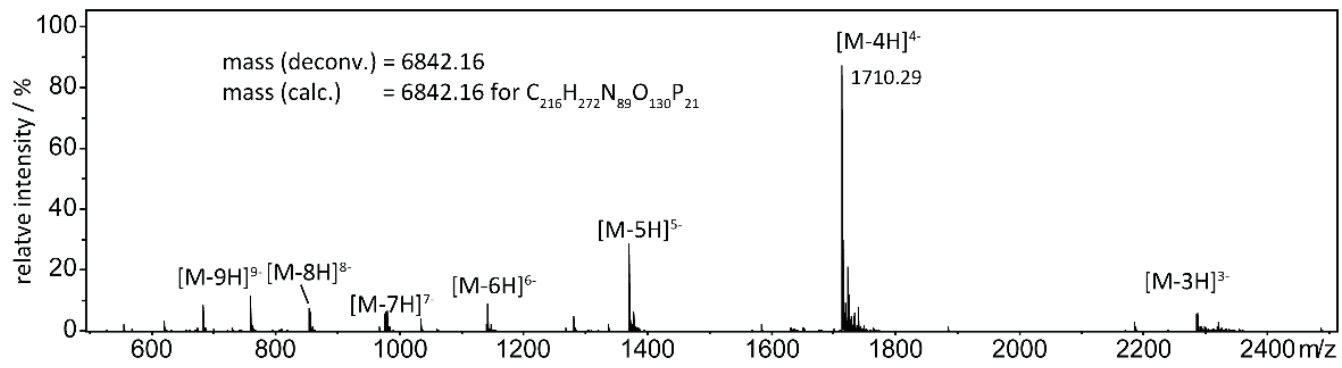

$92 L^{R}$ 


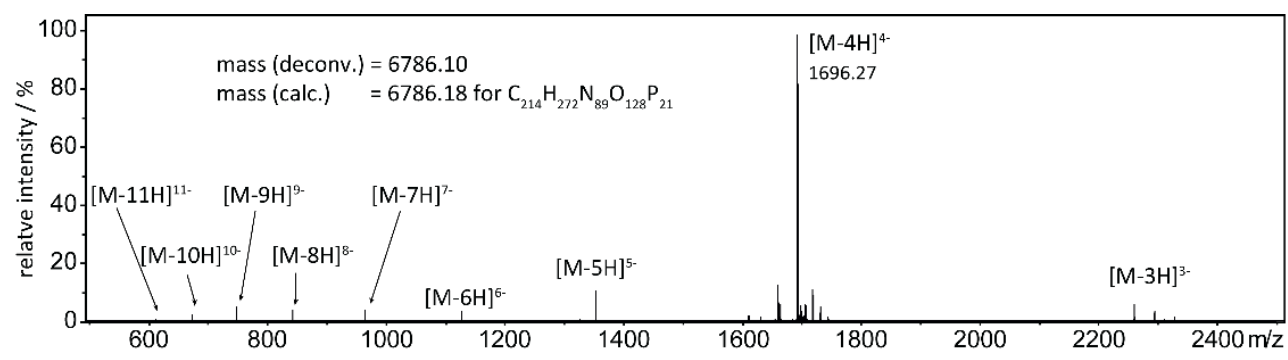

htelL ${ }_{3}^{s} \mathrm{M}$

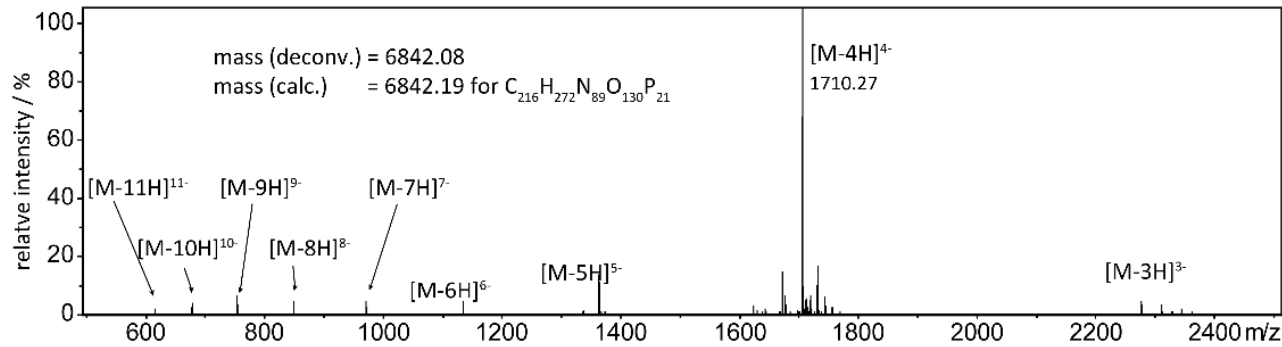

htelL ${ }_{2} \mathrm{~K}$

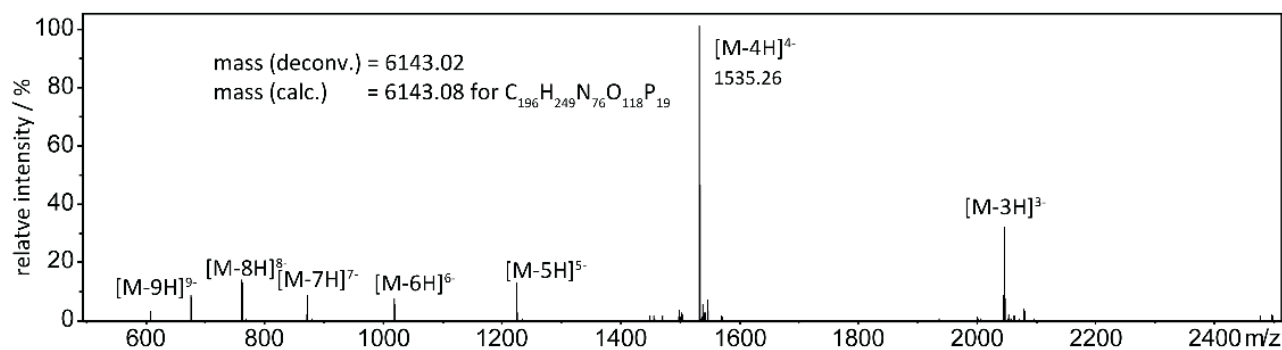

htell ${ }_{2}^{s} \mathrm{~L}$

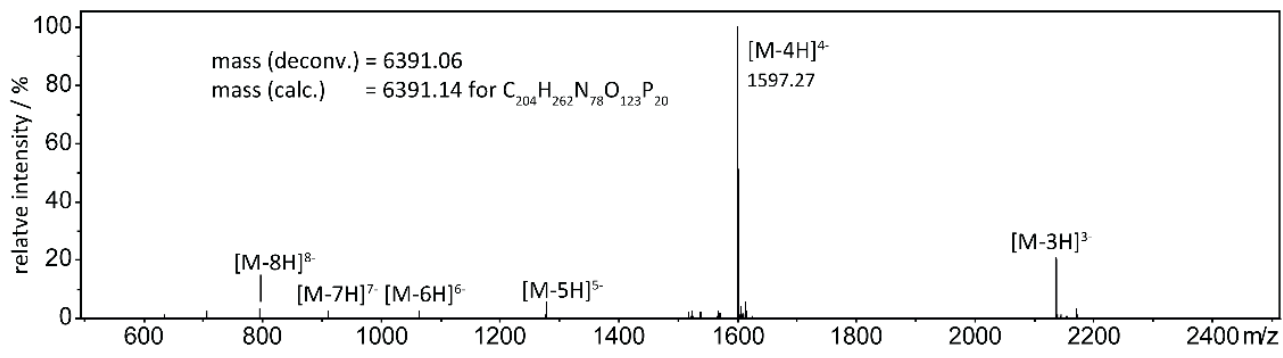

htelL ${ }_{3} \mathrm{~N}$

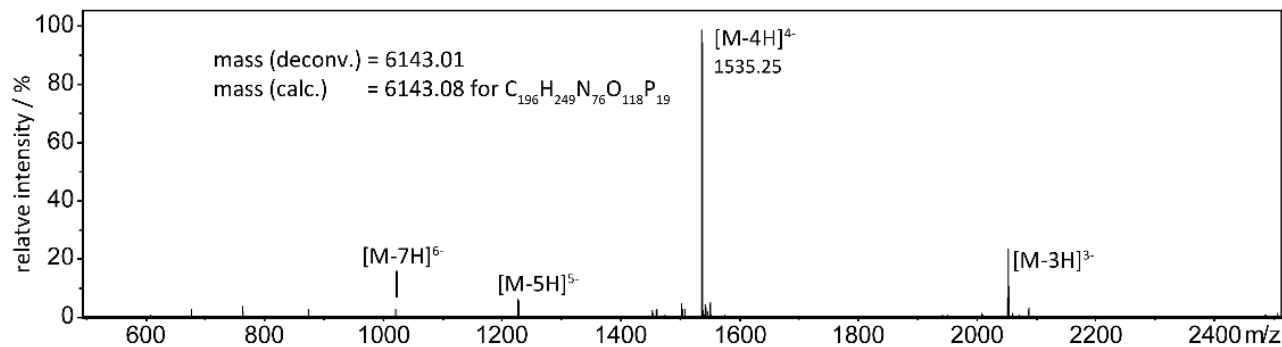

htelL ${ }_{2} \mathrm{M}$ 


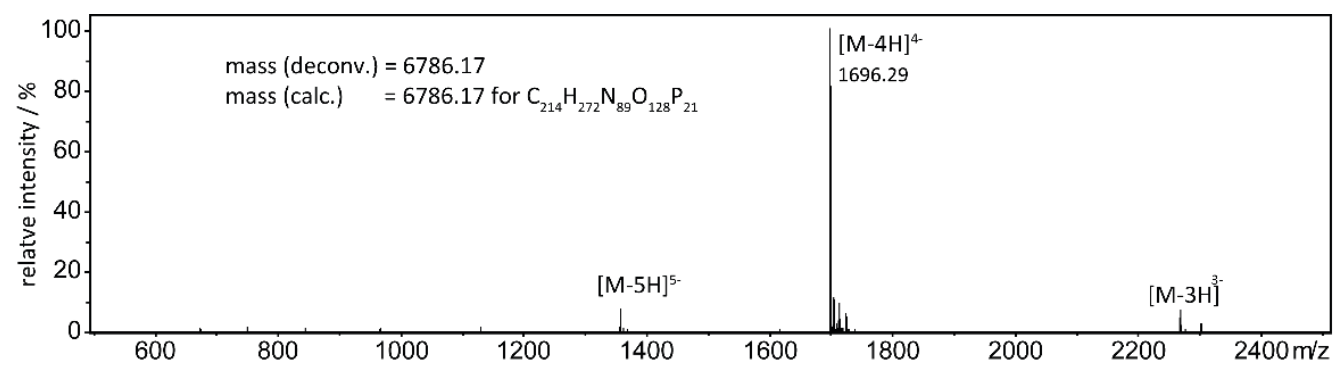

htelL ${ }^{R}{ }_{3}$ I

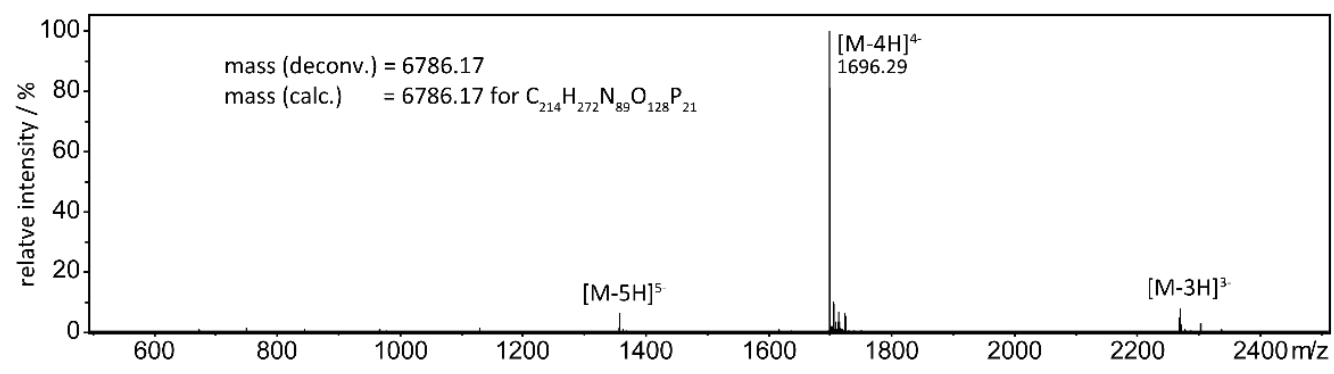

htell ${ }^{R}{ }_{3} \mathrm{~J}$

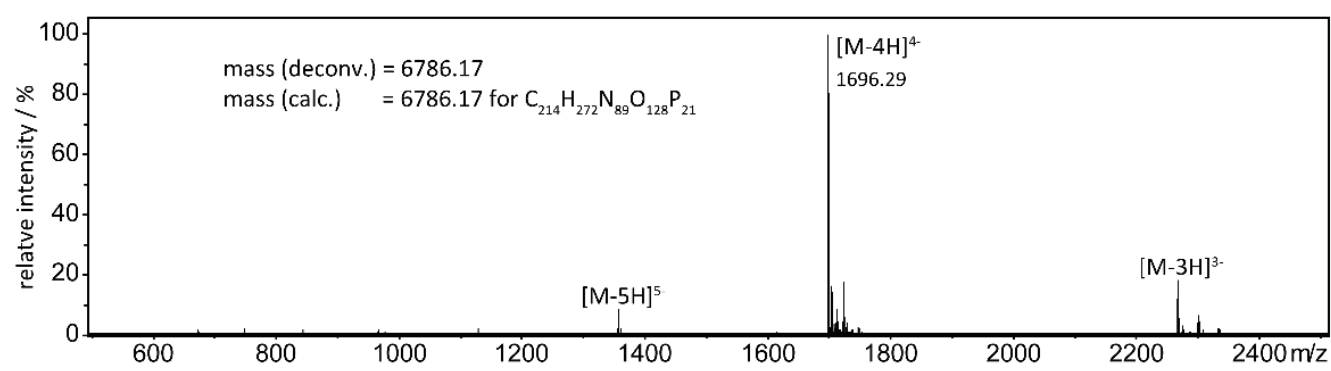

htelL ${ }^{R}{ }_{3} \mathrm{~K}$

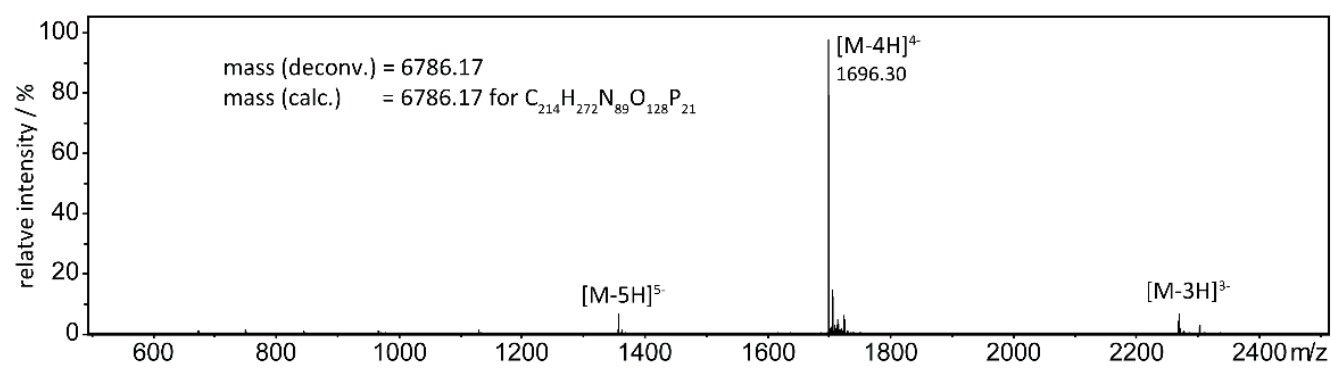

htell ${ }^{R}{ }_{3} \mathrm{~L}$

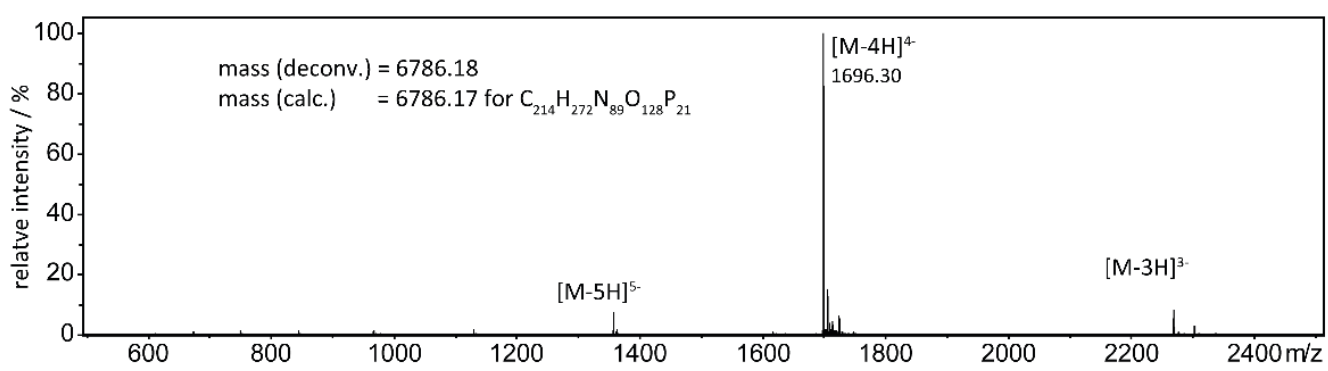

htelL ${ }_{3}^{R} \mathrm{M}$ 


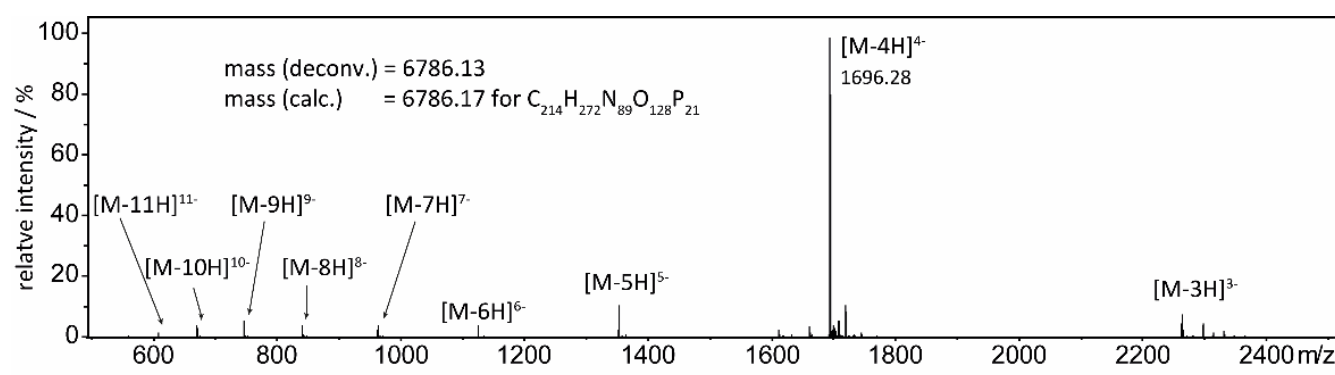

\section{htelL ${ }_{3}^{s} \mathrm{H}$}

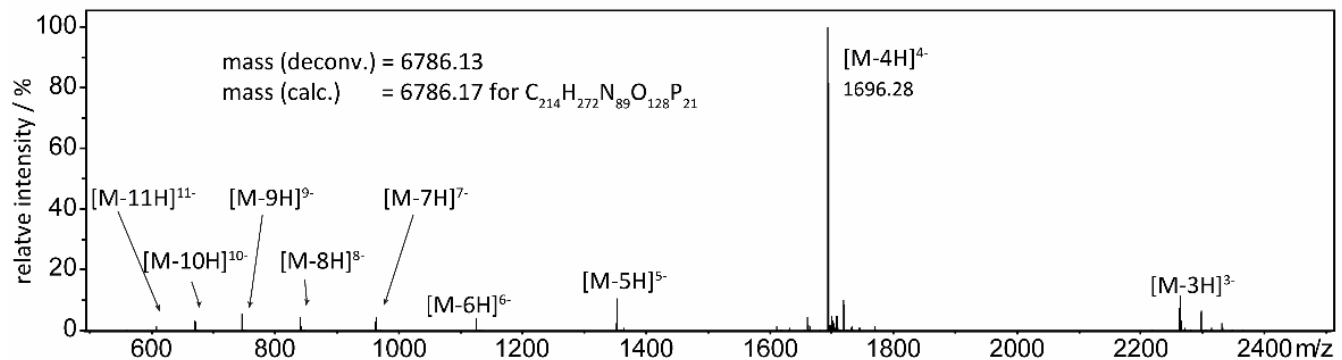

\section{htelL ${ }_{3} \mathbf{I}$}

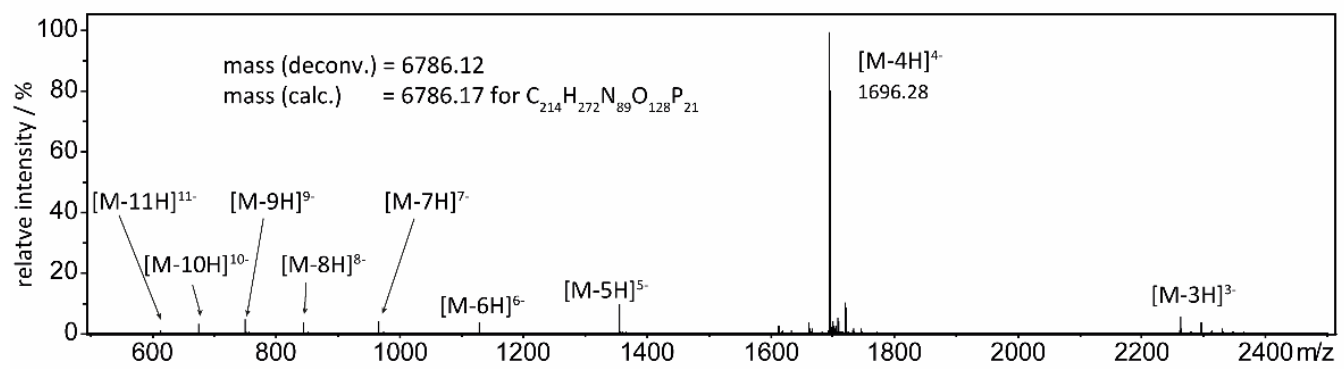

htelL $s_{3} \mathrm{~J}$

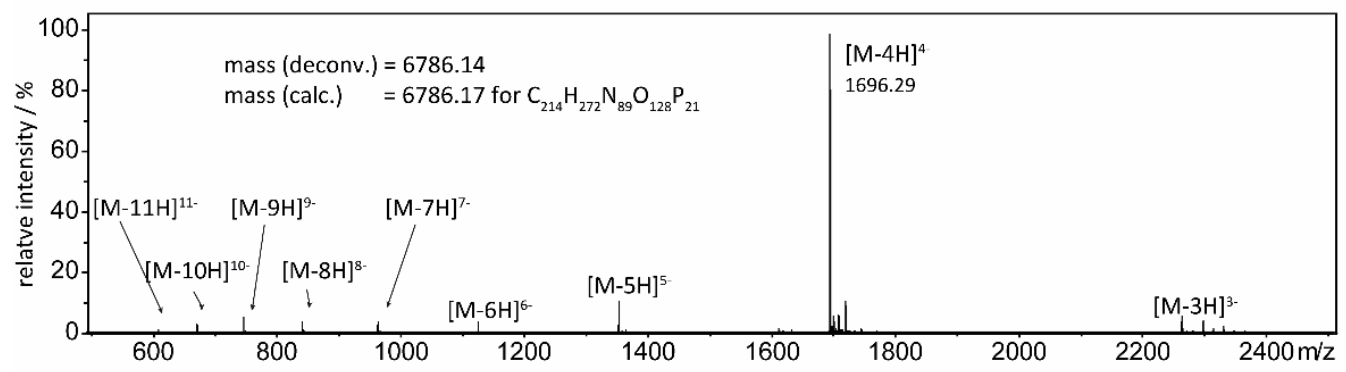

htelL ${ }_{3}{ }_{3} \mathrm{~K}$

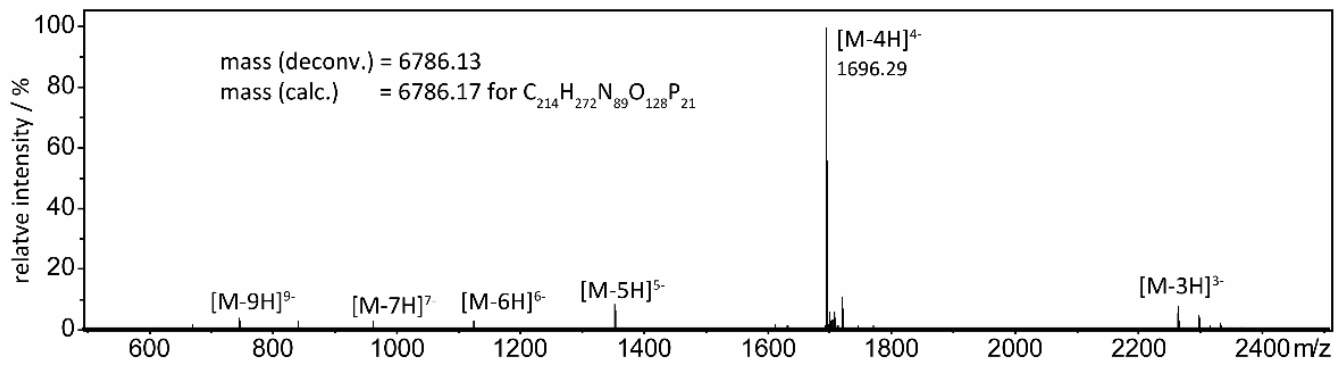

htelL ${ }_{3} \mathrm{~L}$ 


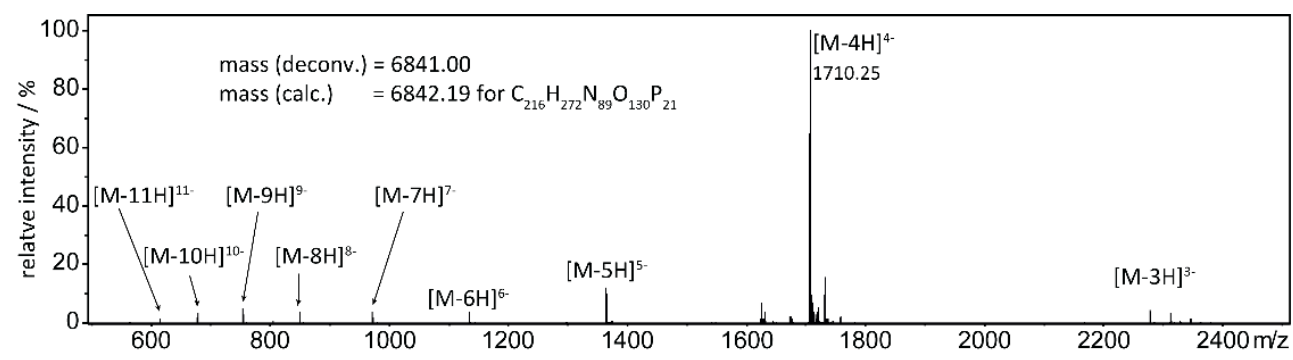

htelL ${ }_{2} G$

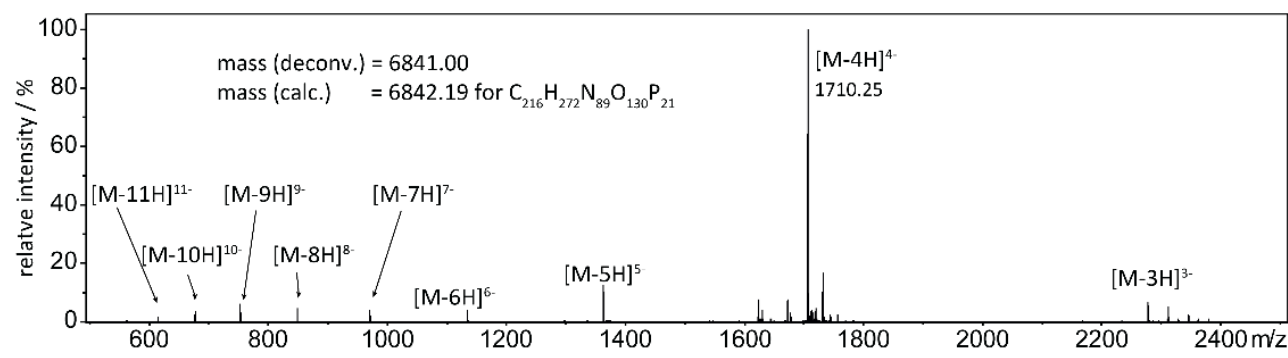

htelL ${ }_{2} \mathrm{H}$

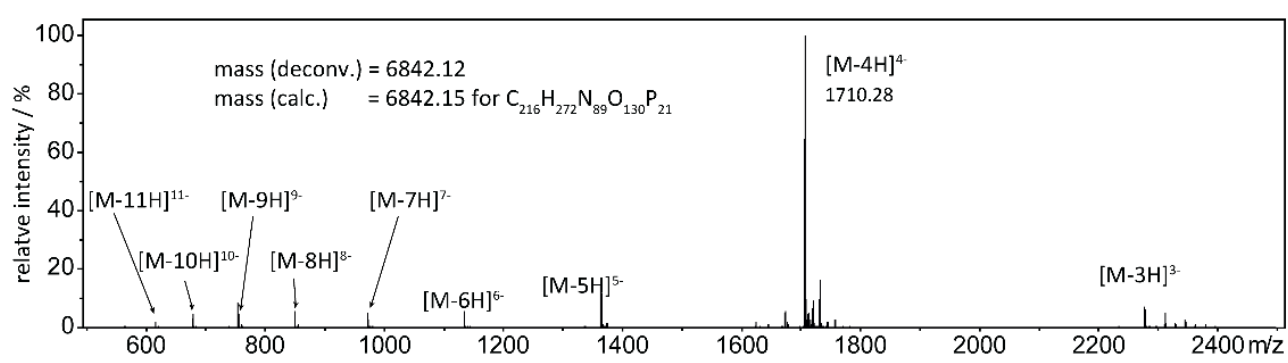

htelL ${ }_{2} \mathbf{I}$

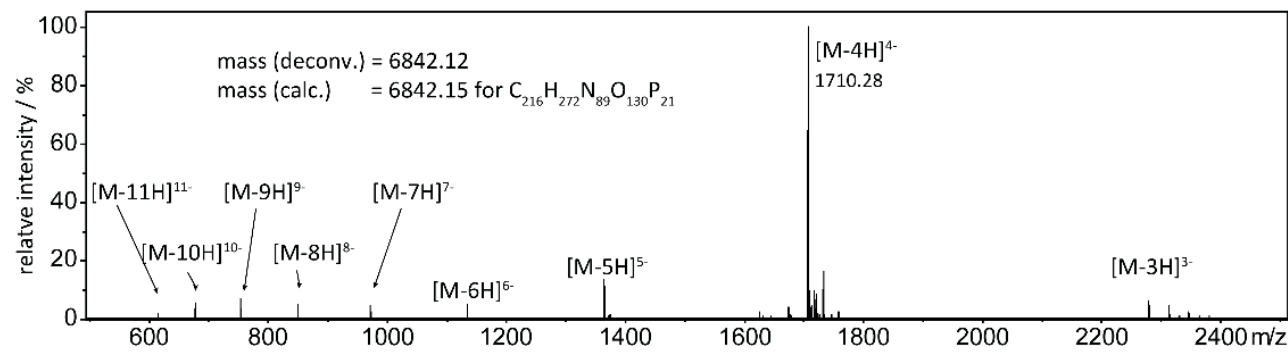

htelLs ${ }_{2} \mathrm{~J}$

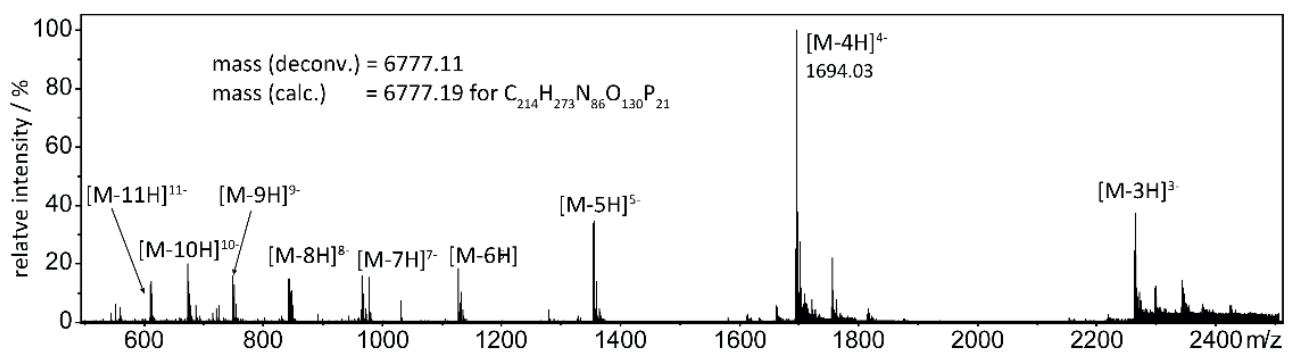

htelL ${ }_{3}^{s} \mathrm{G}$ 


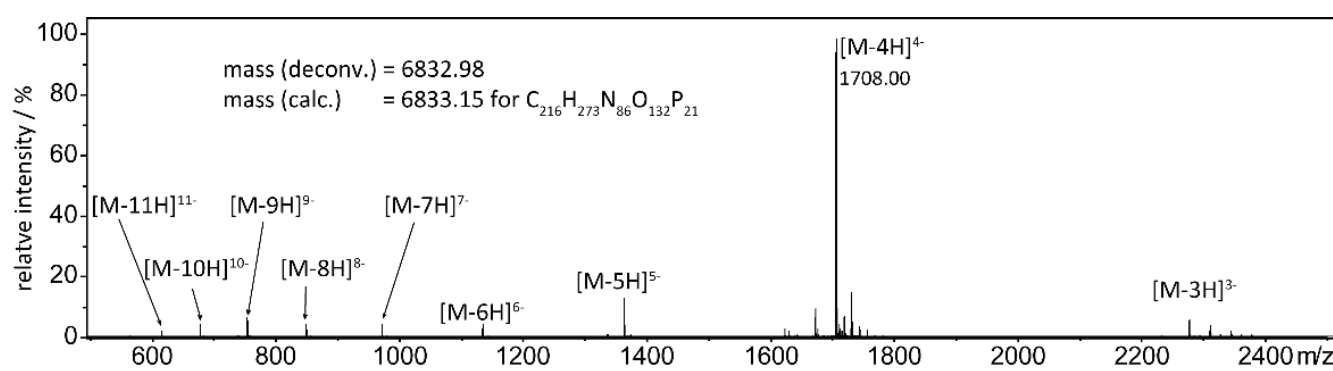

htelL ${ }_{2}^{s} \mathrm{C}$

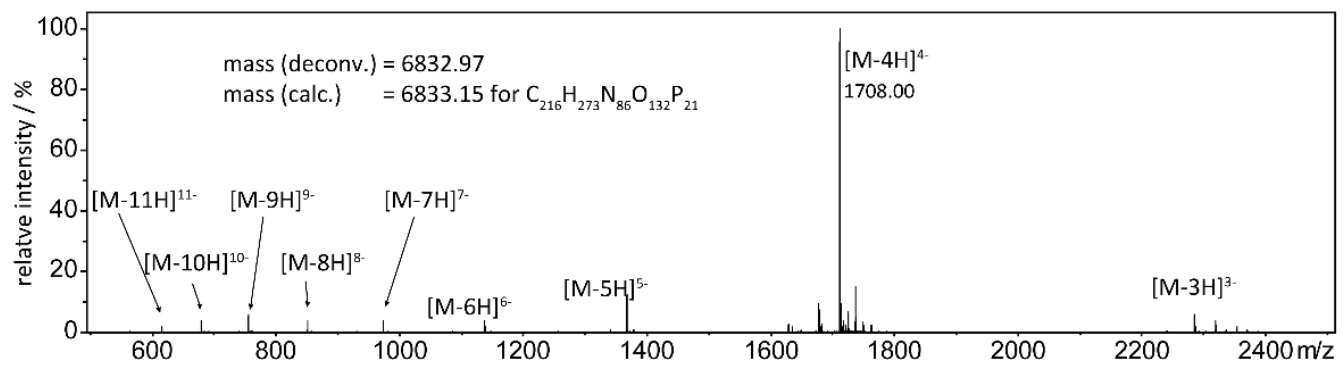

htelL ${ }_{2}{ }_{2} \mathrm{D}$

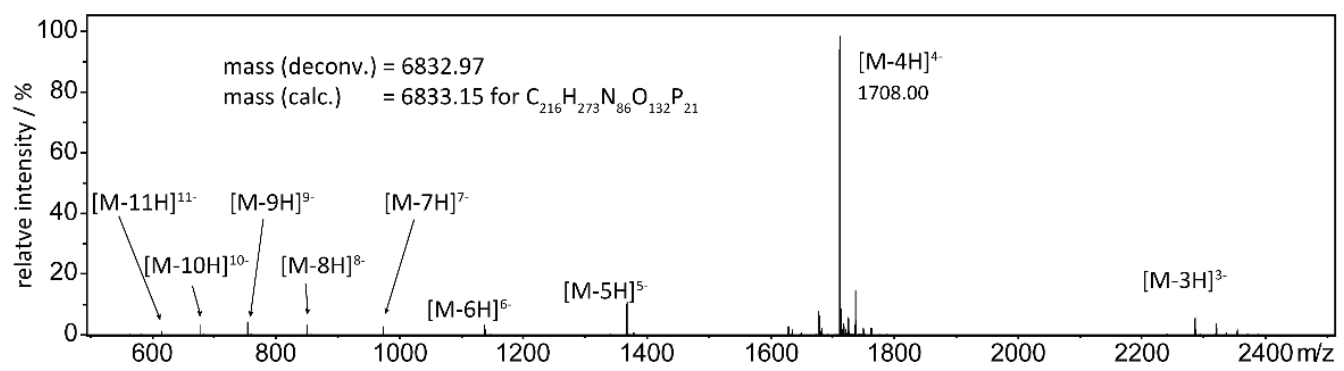

htelL ${ }_{2}{ }_{2} \mathrm{~A}$

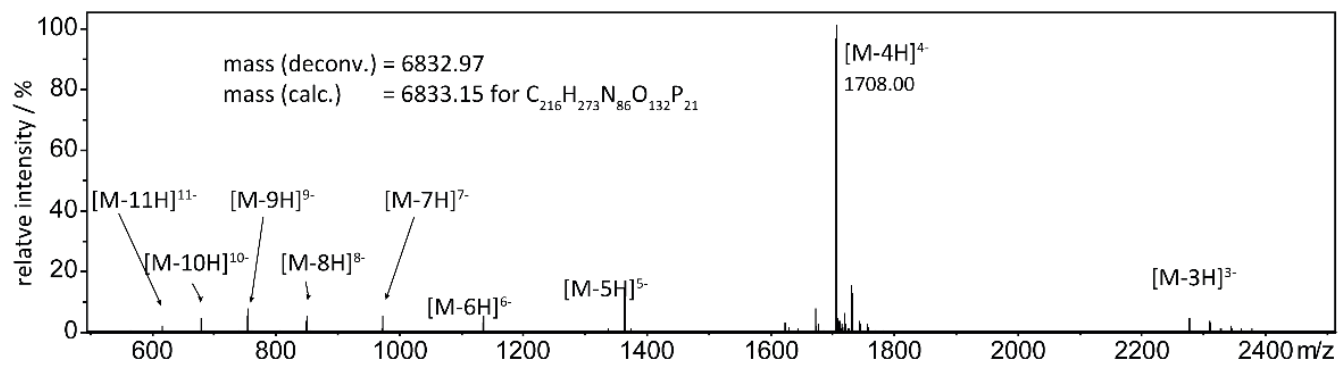

htelL ${ }_{2} \mathrm{E}$

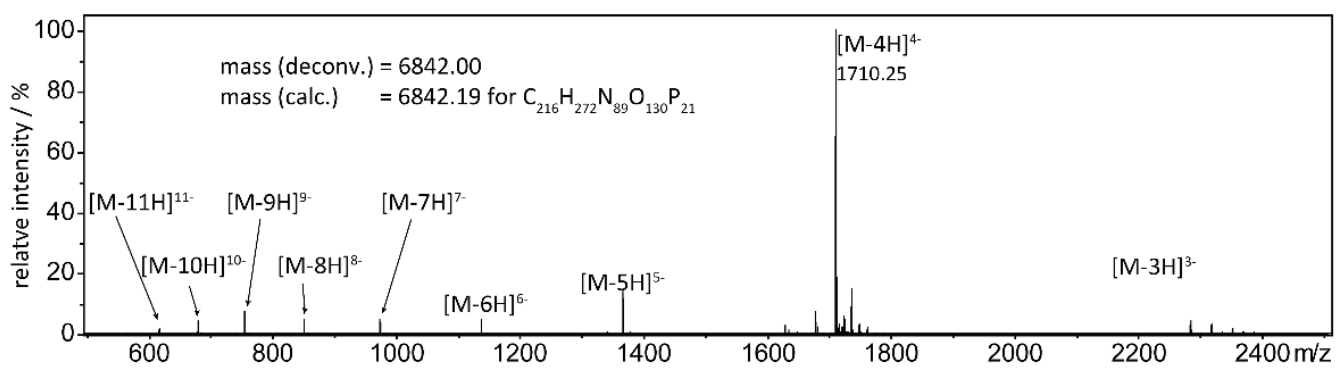

htelLs ${ }_{2} \mathrm{~F}$ 


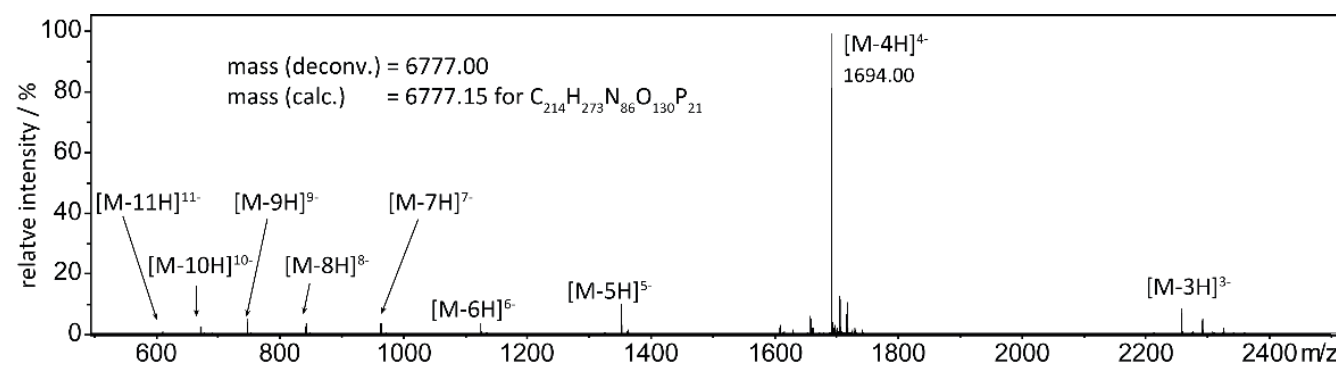

htelL ${ }_{3}^{\mathrm{s}} \mathrm{A}$

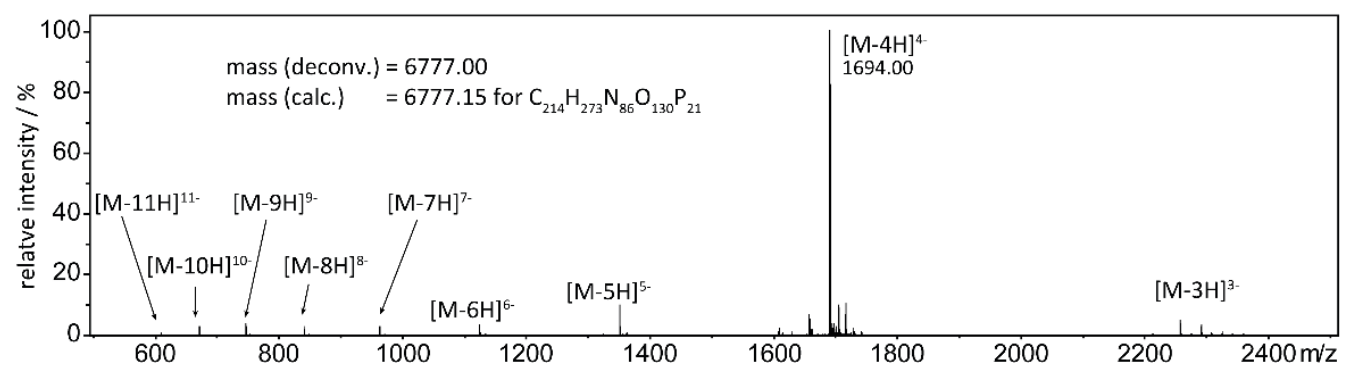

htelL ${ }_{3}^{s} \mathrm{C}$

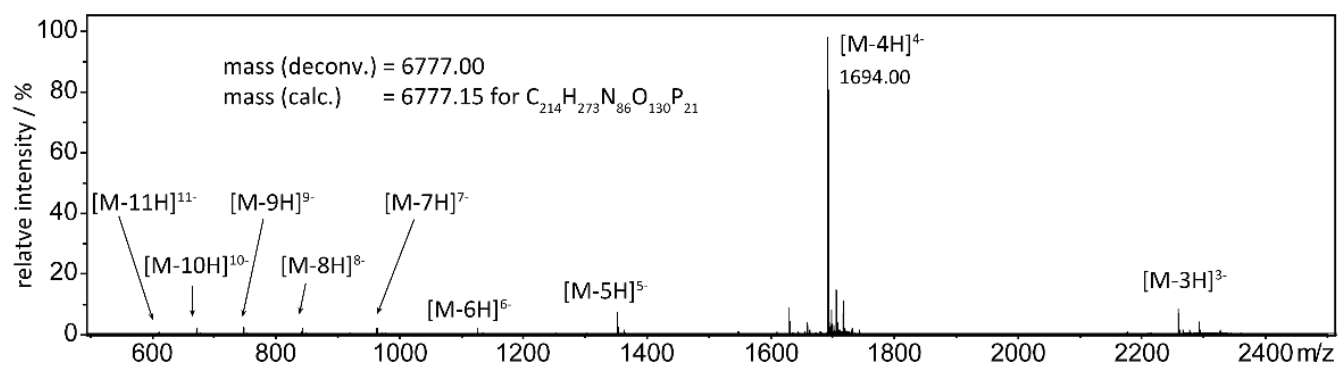

htelL ${ }_{3}^{s} \mathrm{D}$

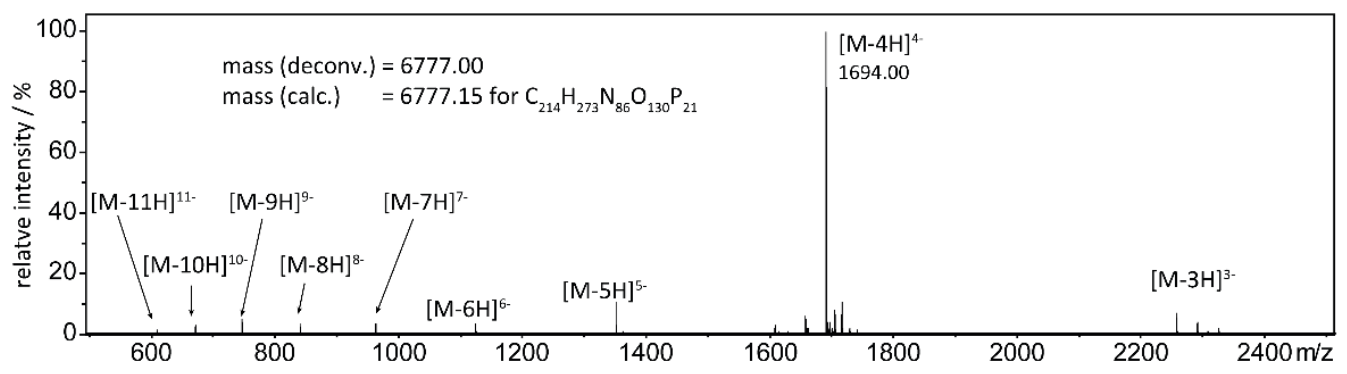

htelL ${ }_{3} \mathrm{E}$

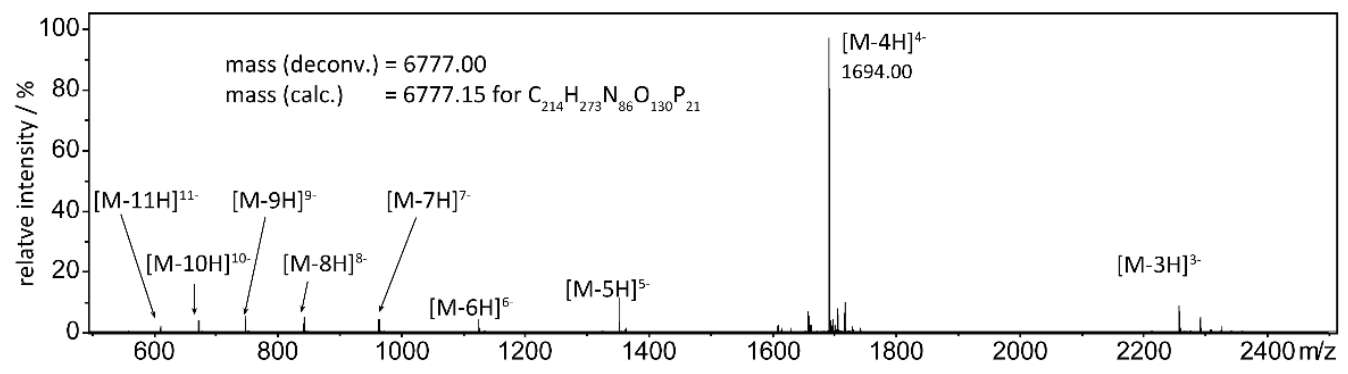

htelL ${ }_{3} \mathrm{~F}$ 


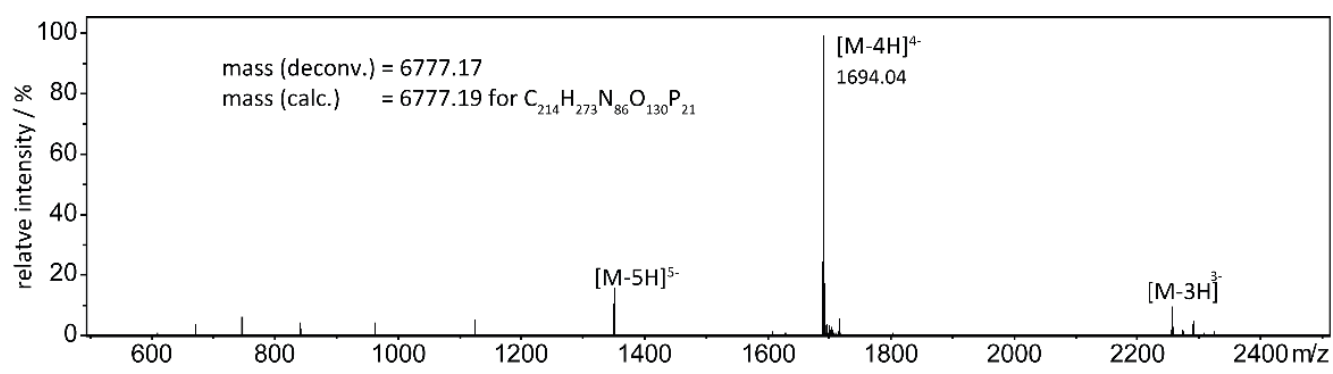

htelL ${ }_{3}^{R} \mathrm{~B}$

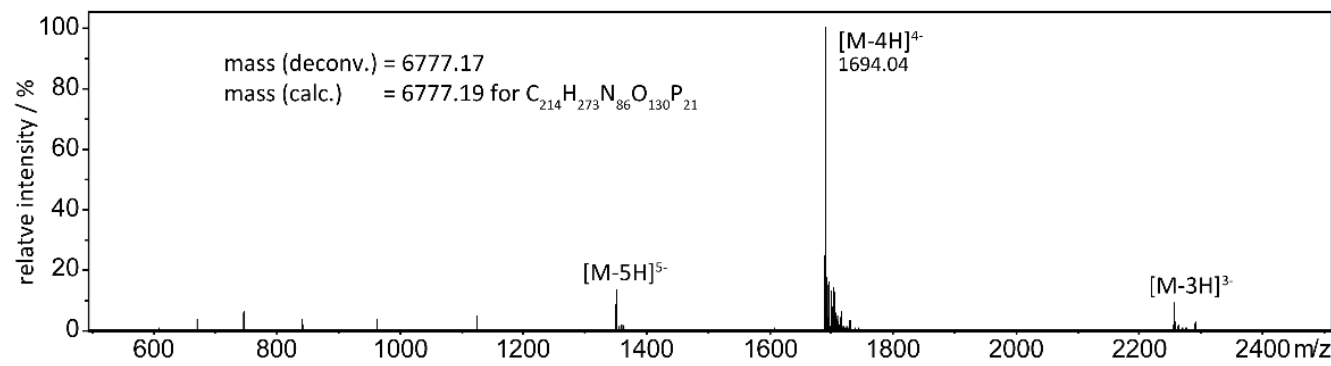

htelL ${ }_{3}^{R} \mathrm{D}$

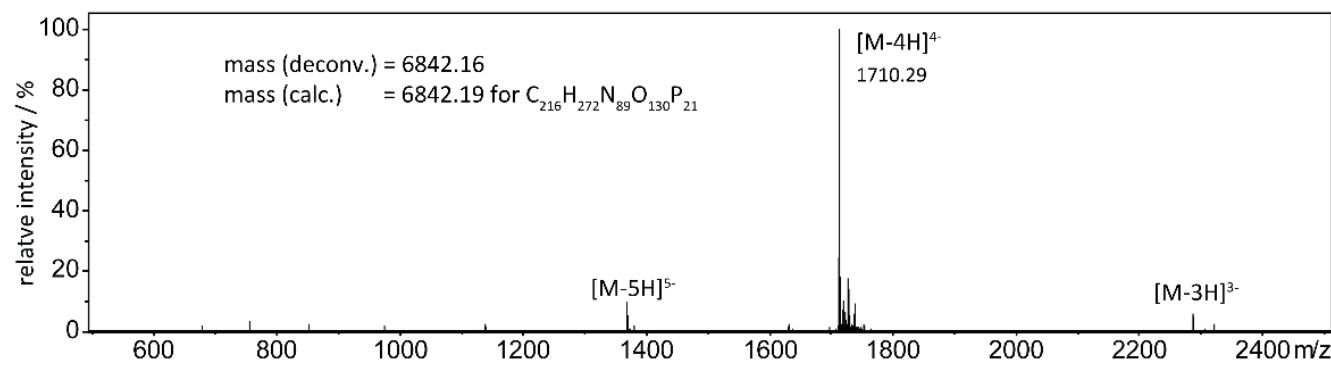

htelL ${ }_{2}^{R} \mathrm{~F}$

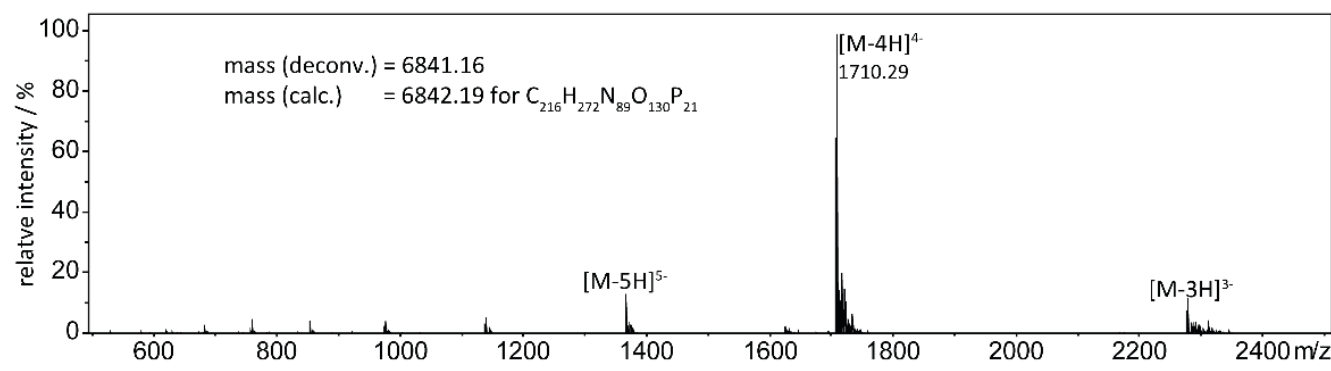

htelL ${ }_{2}^{R} \mathrm{G}$

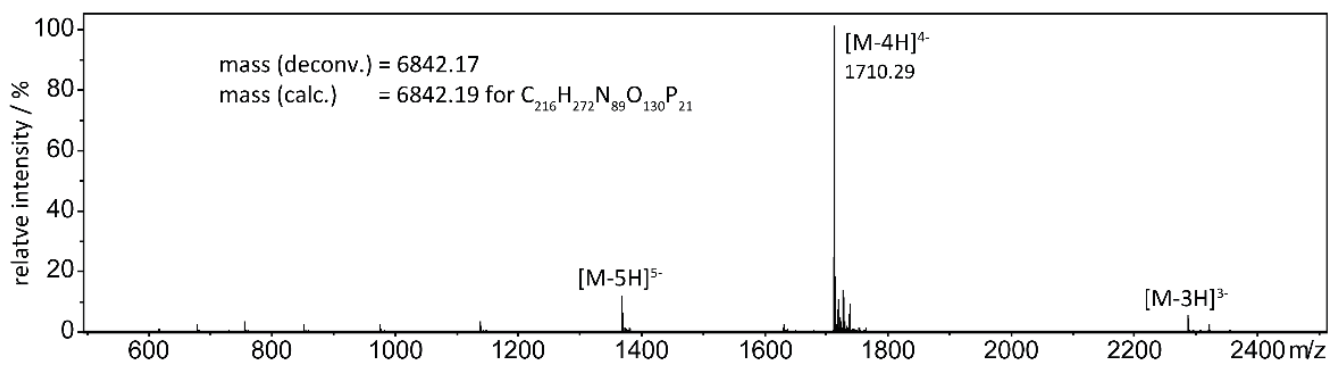

htelL ${ }_{2}^{R} \mathrm{H}$

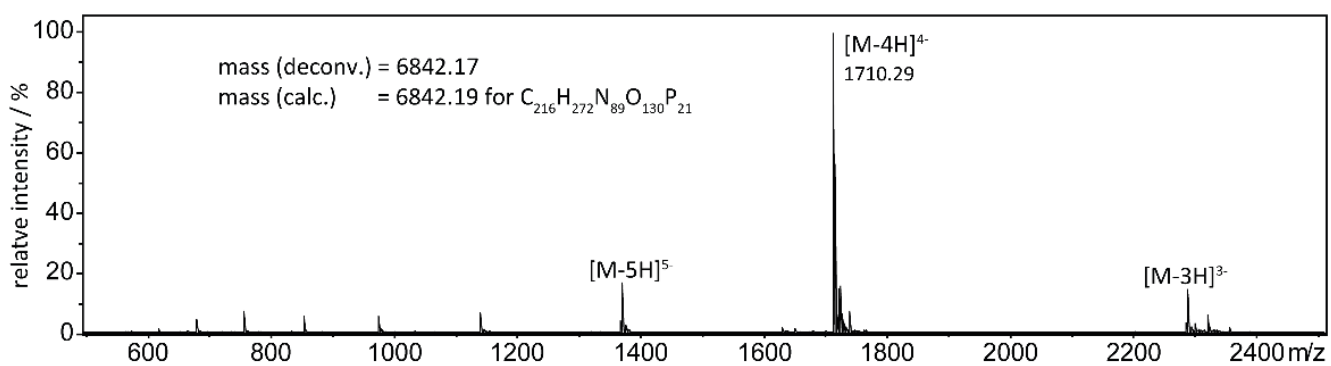

htelL ${ }_{2}^{R} \mid$ 


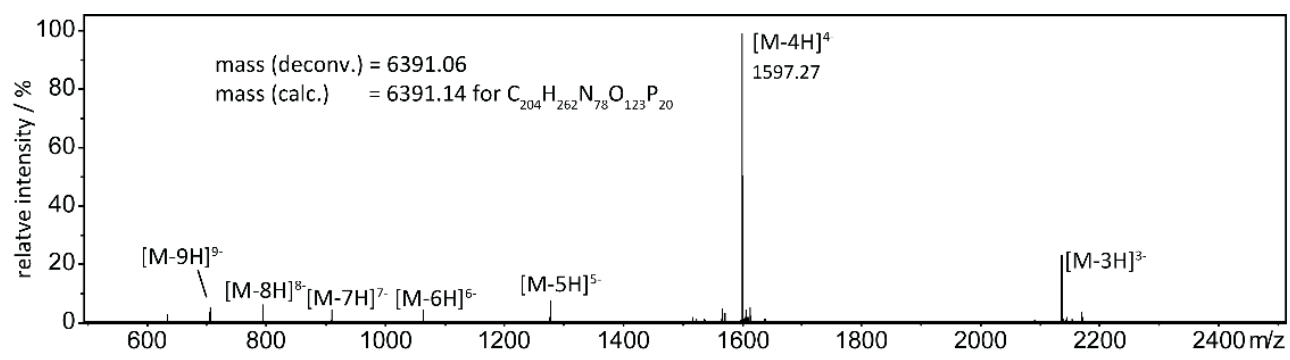

htelLs ${ }_{3} \mathrm{O}$

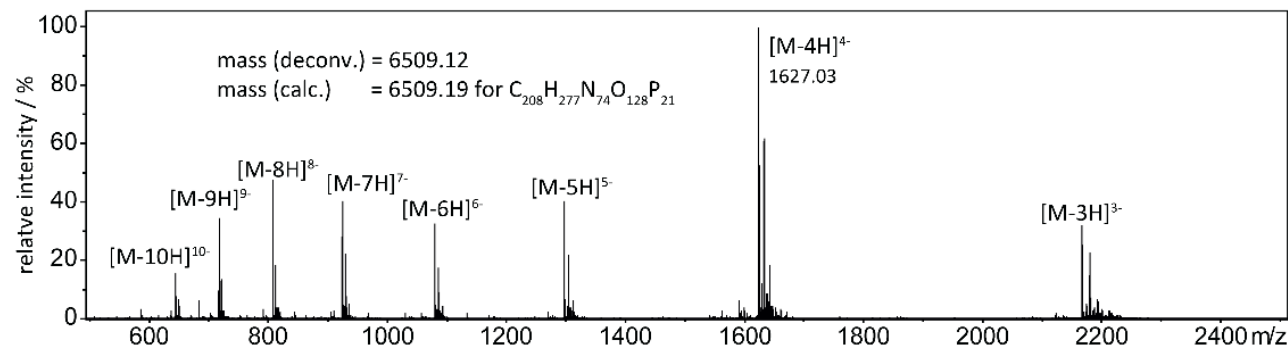

htelLs $_{6}$

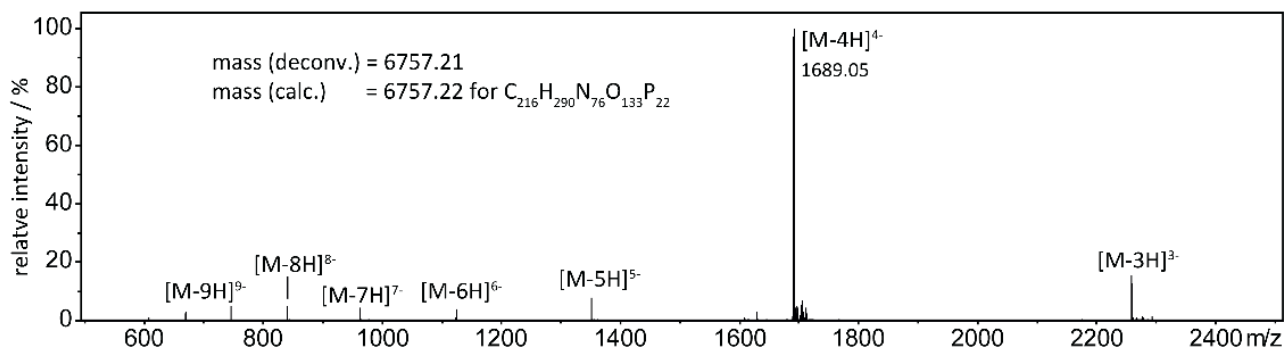

htelLs $_{7}$

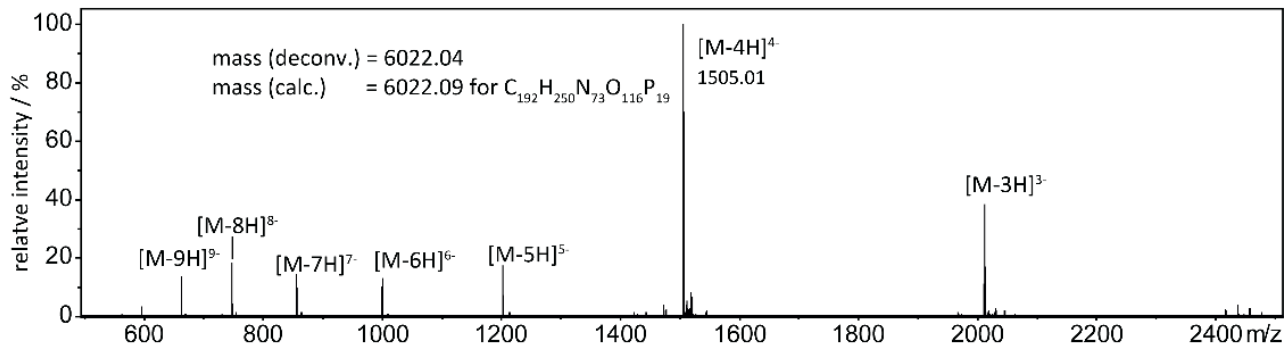

htelLs ${ }_{4} \mathrm{~B}$

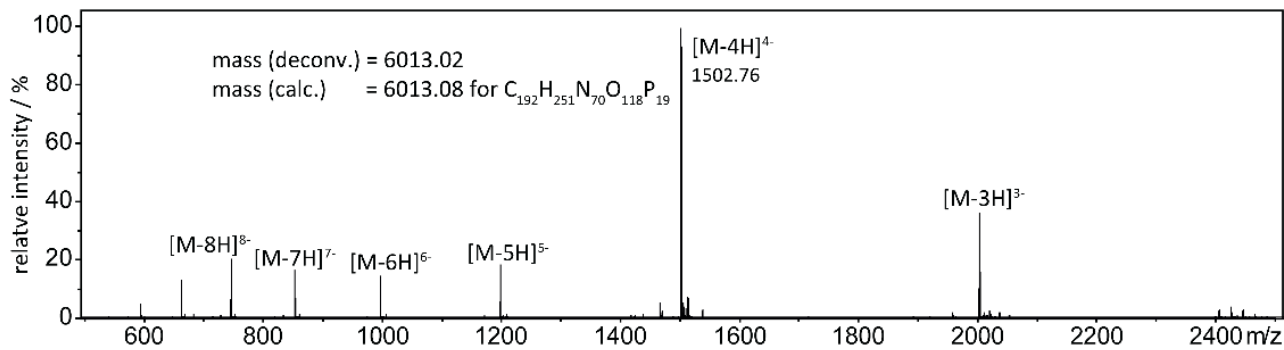

htelLs ${ }_{4} \mathrm{C}$ 


\section{Analytical RP-HPLC}

To check the purity of the synthesized and purified oligonucleotides, samples $(10 \mu \mathrm{L})$ with DNA concentrations of around $500 \mu \mathrm{M}$ in $20 \mathrm{mM}$ TMAA pH 7 or TEAA pH 7 were prepared and analytical RP-HPLC was performed on an Agilent Technologies 1260 Infinity II system equipped with an autosampler, column oven, DAD detector and a Macherey-Nagel EC 250/4.6 NUCLEODUR 100-5 C18ec column (oven temperature: $60^{\circ} \mathrm{C}$, flow rate: $0.75 \mathrm{~mL} / \mathrm{min}$ or $1.00 \mathrm{~mL} / \mathrm{min}$, solvent A: $50 \mathrm{mM}$ TEAA pH 7, solvent B: 70:30 MeCN/50 mM TEAA pH 7). The RP-HPLC traces including the used solvent gradient are shown below.
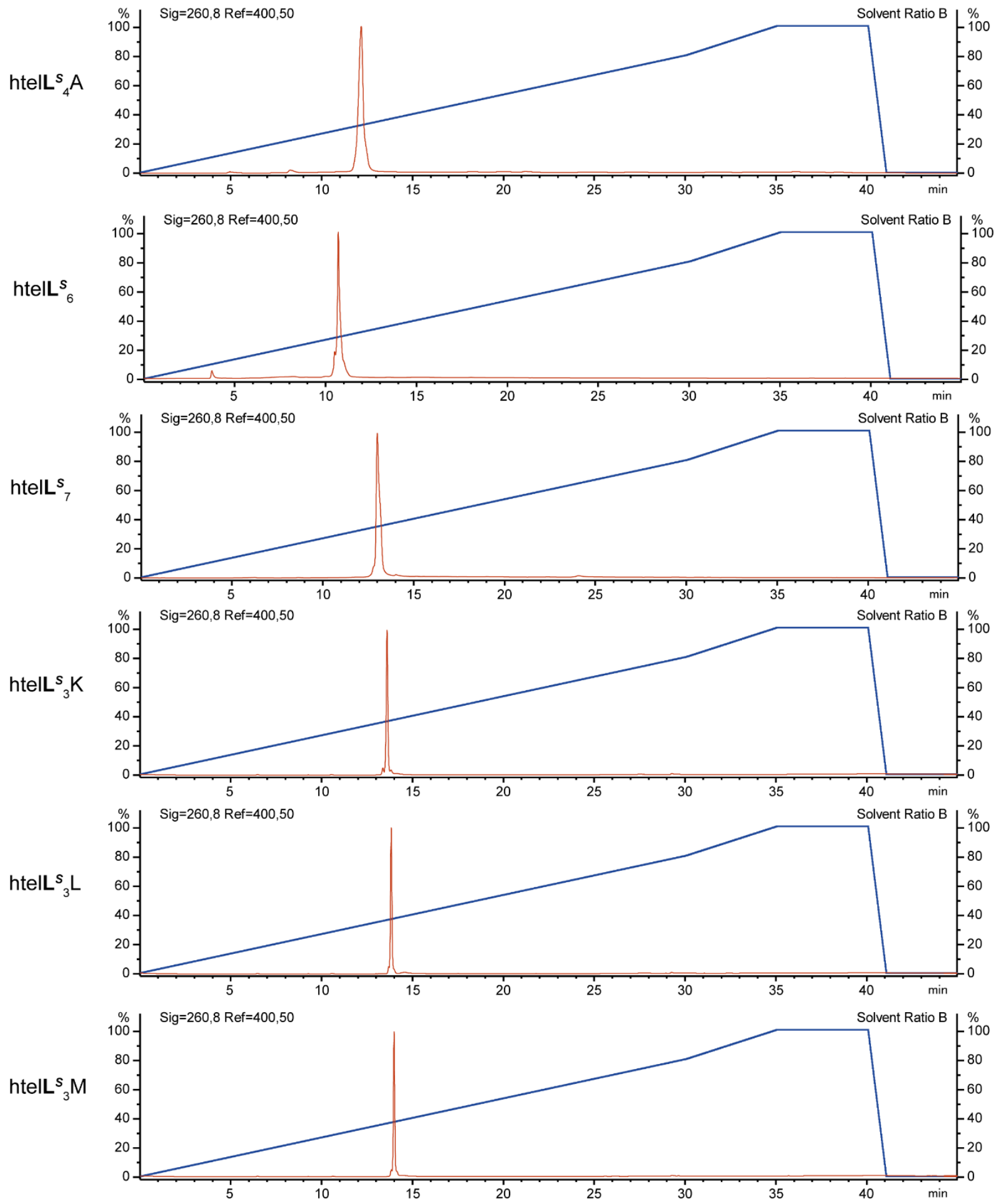

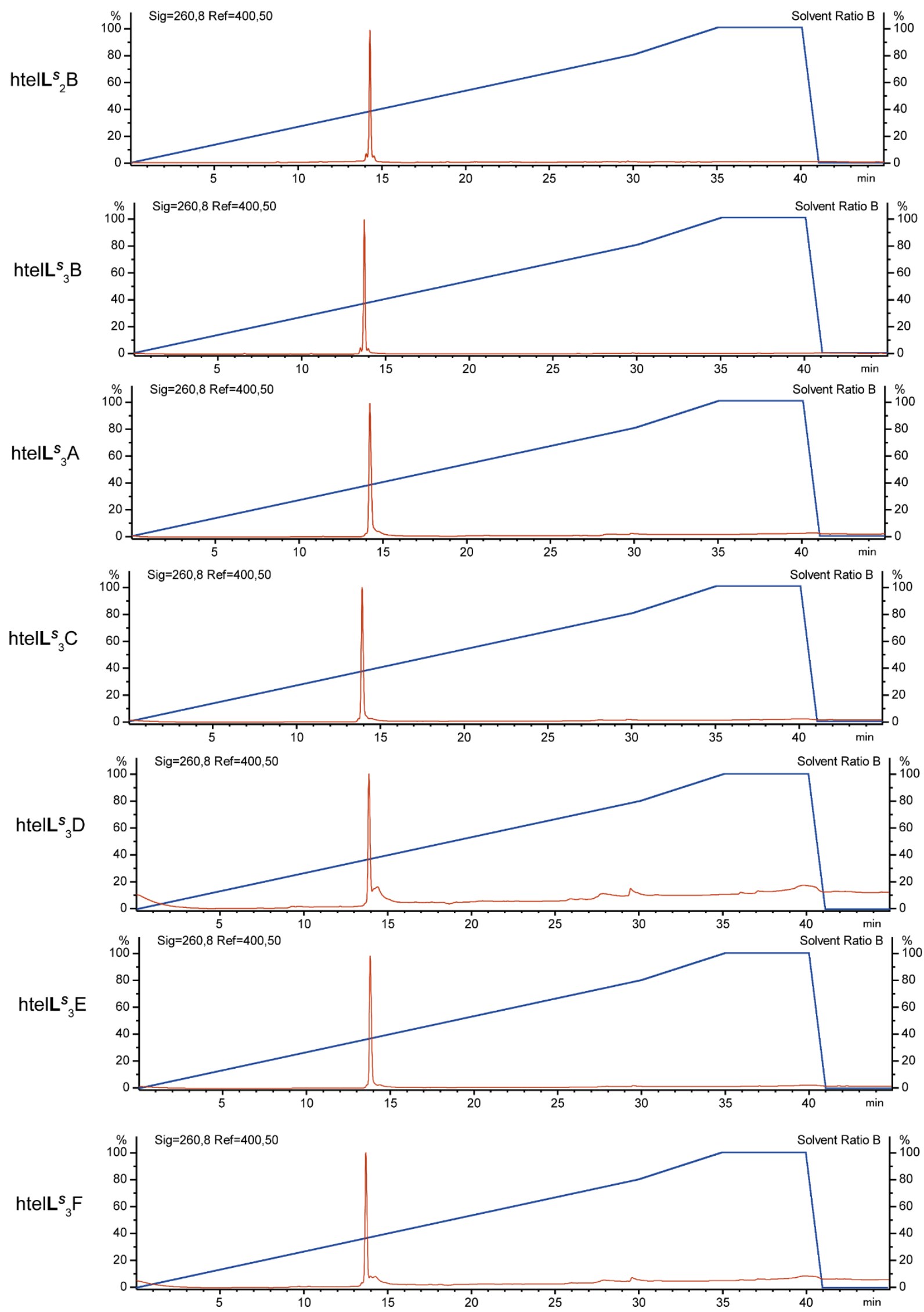

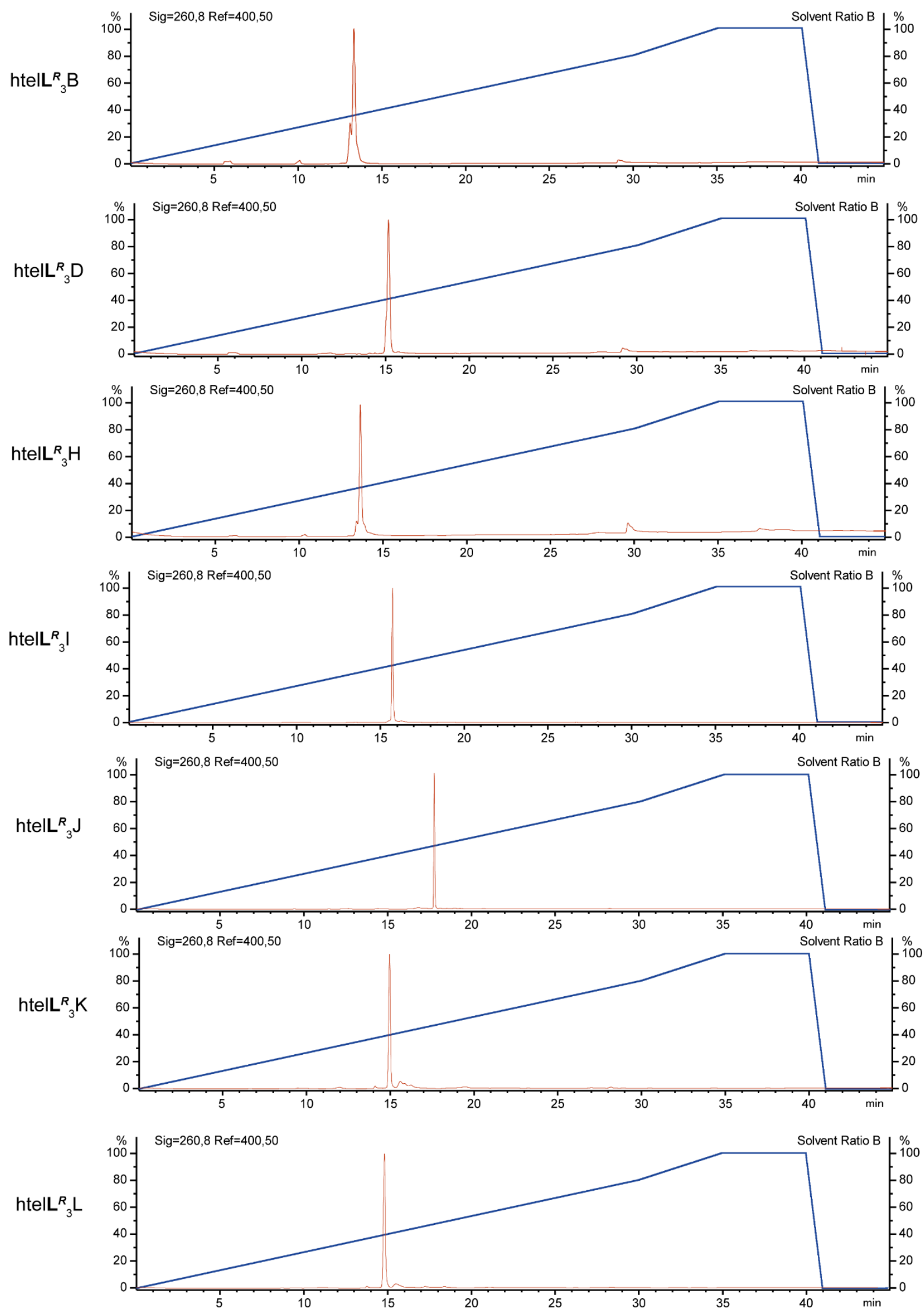

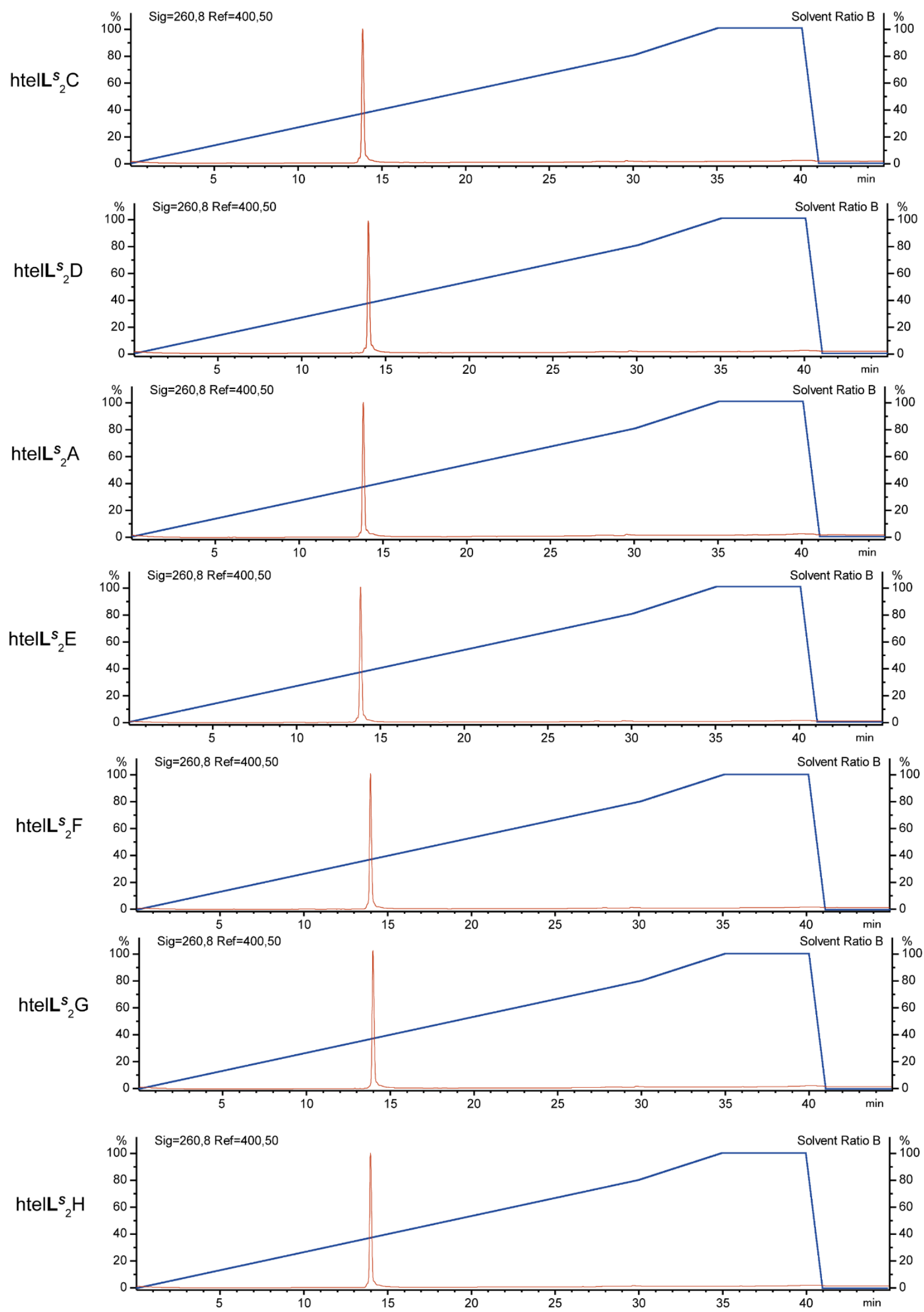


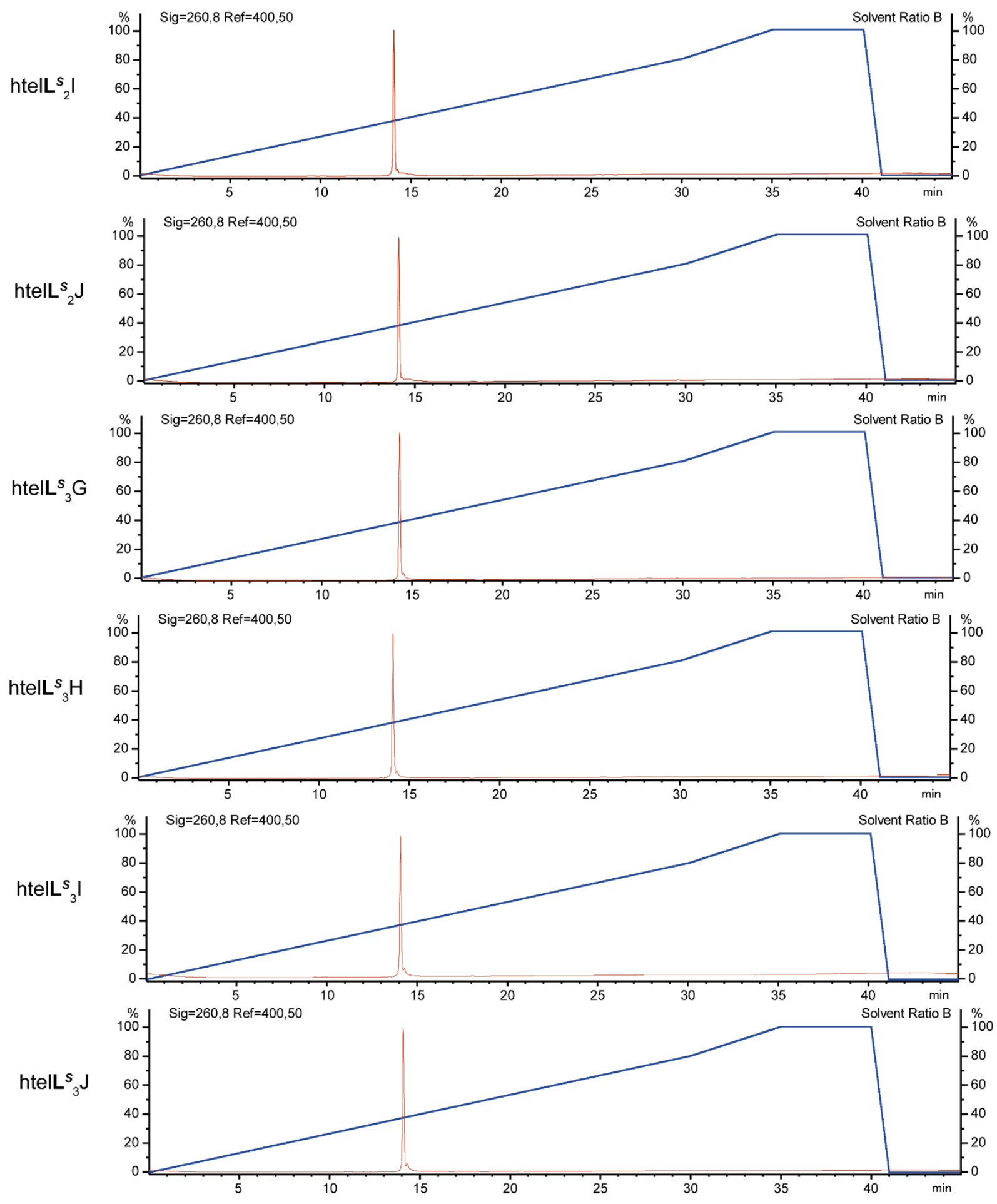



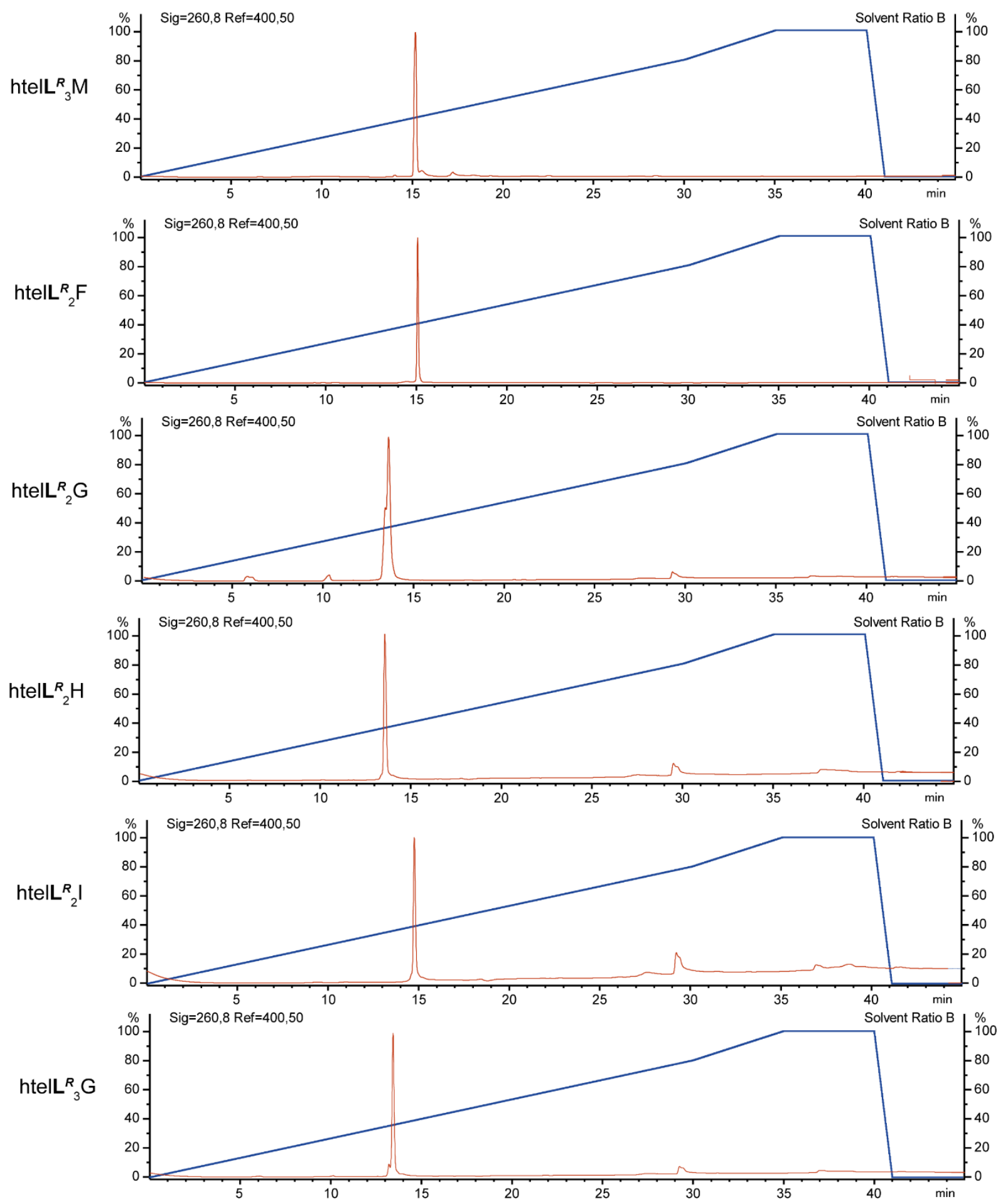

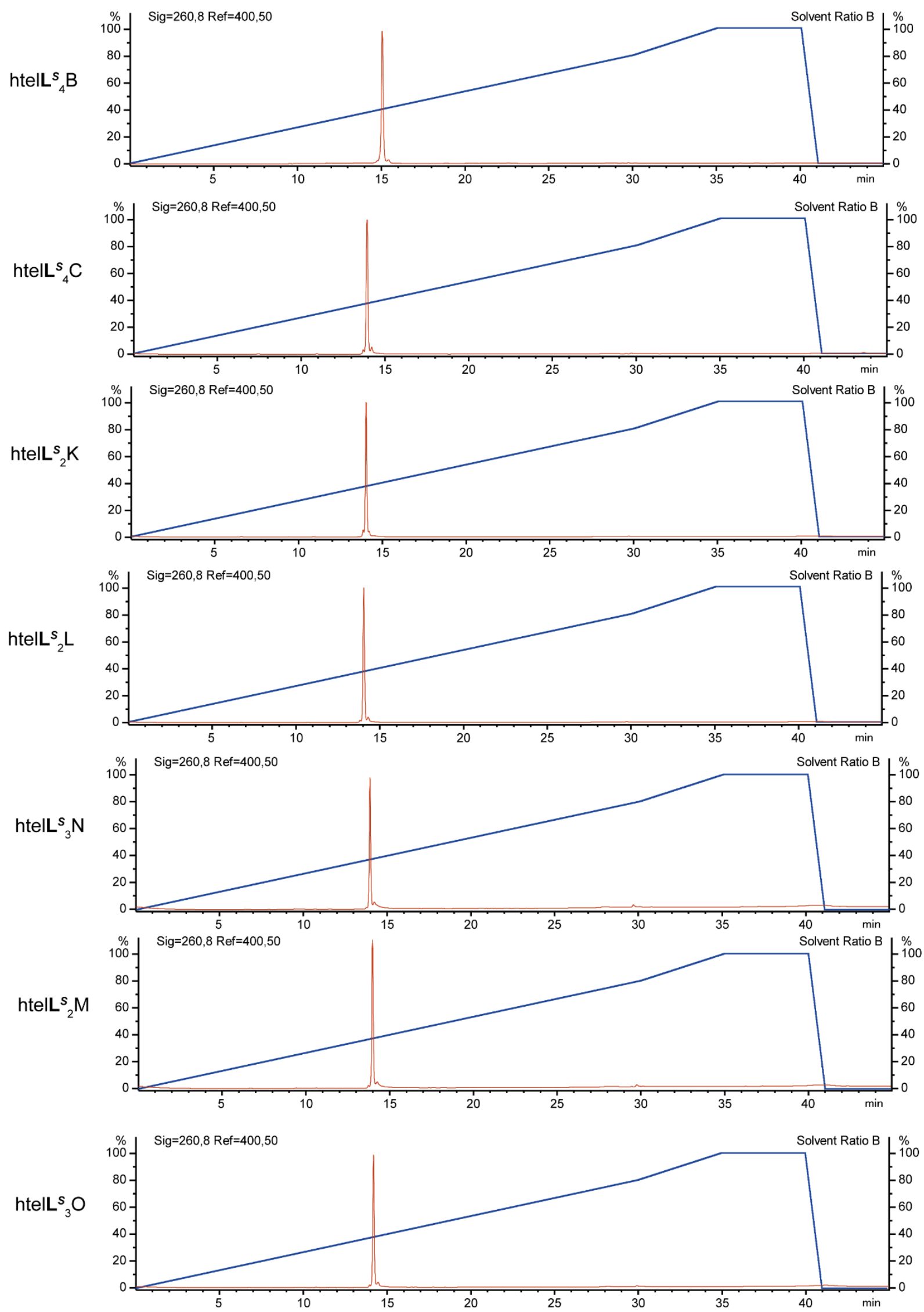


\section{References:}

[1] P. M. Punt, G. H. Clever, Chem. - Eur. J. 2019, 25, 13987-13993.

[2] E. B. Mubofu, J. B. F. N. Engberts, J. Phys. Org. Chem. 2004, 17, 180-186.

[3] J. Wang, E. Benedetti, L. Bethge, S. Vonhoff, S. Klussmann, J. Vasseur, J. Cossy, M.

Smietana, S. Arseniyadis, Angew. Chem. Int. Ed. 2013, 52, 11546-11549.

[4] N. Duchemin, M. Cattoen, O. Gayraud, S. Anselmi, B. Siddiq, R. Buccafusca, M. Daumas, V.

Ferey, M. Smietana, S. Arseniyadis, Org. Lett. 2020, 22, 5995-6000.

[5] M. C. Myers, A. R. Bharadwaj, B. C. Milgram, K. A. Scheidt, J. Am. Chem. Soc. 2005, 127, $14675-14680$.

[6] S. Otto, F. Bertoncin, J. B. F. N. Engberts, J. Am. Chem. Soc. 1996, 118, 7702-7707.

[7] D. M. Engelhard, R. Pievo, G. H. Clever, Angew. Chem. Int. Ed. 2013, 52, 12843-12847.

[8] D. M. Engelhard, J. Nowack, G. H. Clever, Angew. Chem. Int. Ed. 2017, 56, 11640-11644.

[9] D. Coquière, B. L. Feringa, G. Roelfes, Angew. Chem. Int. Ed. 2007, 46, 9308-9311.

[10] V. D’Atri, M. Porrini, F. Rosu, V. Gabelica, J. Mass Spectrom. 2015, 50, 711-726.

[11] A. Marchand, V. Gabelica, J. Am. Soc. Mass Spectr. 2014, 25, 1146-1154.

[12] S. Pronk, S. Páll, R. Schulz, P. Larsson, P. Bjelkmar, R. Apostolov, M. R. Shirts, J. C.

Smith, P. M. Kasson, D. van der Spoel, B. Hess, E. Lindahl, Bioinformatics 2013, 29, 845-54.

[13] B. Hess, C. Kutzner, D. van der Spoel, E. Lindahl, J. Chem. Theory Comput. 2008, 4, 43547.

[14] D. V. D. Spoel, E. Lindahl, B. Hess, G. Groenhof, A. E. Mark, H. J. C. Berendsen, J. Comput. Chem. 2005, 26, 1701-1718.

[15] M. J. Abraham, T. Murtola, R. Schulz, S. Páll, J. C. Smith, B. Hess, E. Lindahl, Softwarex 2015, 1-2, 19-25.

[16] P. Szilárd, M. J. Abraham, C. Kutzner, B. Hess, E. Lindahl, 2015, 3-27.

[17] B. Hess, H. Bekker, H. J. C. Berendsen, J. G. E. M. Fraaije, J. Comput. Chem. 1997, 18, $1463-1472$.

[18] G. Bussi, D. Donadio, M. Parrinello, J. Chem. Phys. 2007, 126, 014101.

[19] I. Ivani, P. D. Dans, A. Noy, A. Pérez, I. Faustino, A. Hospital, J. Walther, P. Andrio, R. Goñi, A. Balaceanu, G. Portella, F. Battistini, J. L. Gelpí, C. González, M. Vendruscolo, C. A. Laughton, S. A. Harris, D. A. Case, M. Orozco, Nat. Methods 2016, 13, 55-58.

[20] A. Pérez, I. Marchán, D. Svozil, J. Sponer, T. E. Cheatham, C. A. Laughton, M. Orozco, Biophys. J. 2007, 92, 3817-3829.

[21] M. J. Frisch, G. W. Trucks, H. B. Schlegel, G. E. Scuseria, M. A. Robb, J. R. Cheeseman, G. Scalmani, V. Barone, G. A. Petersson, H. Nakatsuji, X. Li, M. Caricato, A. V. Marenich, J.

Bloino, B. G. Janesko, R. Gomperts, B. Mennucci, H. P. Hratchian, J. V. Ortiz, A. F. Izmaylov, J. L. Sonnenberg, D. Williams-Young, F. Ding, F. Lipparini, F. Egidi, J. Goings, B. Peng, A. Petrone, T. Henderson, D. Ranasinghe, V. G. Zakrzewski, J. Gao, N. Rega, G. Zheng, W. Liang, M. Hada, M. Ehara, K. Toyota, R. Fukuda, J. Hasegawa, M. Ishida, T. Nakajima, Y. Honda, O. Kitao, H. Nakai, T. Vreven, K. Throssell, J. A. Montgomery, Jr., J. E. Peralta, F. Ogliaro, M. J. Bearpark, J. J. Heyd, E. N. Brothers, K. N. Kudin, V. N. Staroverov, T. A. Keith, R. Kobayashi, J. Normand, K. Raghavachari, A. P. Rendell, J. C. Burant, S. S. Iyengar, J. Tomasi, M. Cossi, J. M. Millam, M. Klene, C. Adamo, R. Cammi, J. W. Ochterski, R. L. Martin, K. Morokuma, O. Farkas, J. B. Foresman, and D. J. Fox, Gaussian 16, Revision C.01, Gaussian, Inc., Wallingford CT, 2016. 
[22] D. A. Case, I. Y. Ben-Shalom, S. R. Brozell, D. S. Cerutti, T. E. Cheatham, III, V. W. D. Cruzeiro, T. A. Darden, R. E. Duke, D. Ghoreishi, M. K. Gilson, H. Gohlke, A. W. Goetz, D. Greene, R. Harris, N. Homeyer, S. Izadi, A. Kovalenko, T. Kurtzman, T. S. Lee, S. LeGrand, P. Li, C. Lin, J. Liu, T. Luchko, R. Luo, D. J. Mermelstein, K. M. Merz, Y. Miao, G. Monard, C. Nguyen, H. Nguyen, I. Omelyan, A. Onufriev, F. Pan, R. Qi, D. R. Roe, A. Roitberg, C. Sagui, S. Schott-Verdugo, J. Shen, C. L. Simmerling, J. Smith, R. Salomon-Ferrer, J. Swails, R. C. Walker, J. Wang, H. Wei, R. M. Wolf, X. Wu, L. Xiao, D. M. Y. and P. A. Kollman, AMBER 2018, University of California, San Francisco, 2018.

[23] P. Li, K. M. Merz, J. Chem. Inf. Model 2016, 56, 599-604.

[24] M. B. Peters, Y. Yang, B. Wang, L. Füsti-Molnár, M. N. Weaver, K. M. Merz, J. Chem.

Theory Comput. 2010, 6, 2935-2947.

[25] R. Mera-Adasme, K. Sadeghian, D. Sundholm, C. Ochsenfeld, J. Phys. Chem. B 2014, 118, 13106-13111.

[26] P. Li, K. M. Merz, J. Chem. Inf. Model. 2016, 56, 599-604.

[27] A. W. S. da Silva, W. F. Vranken, BMC Res. Notes 2012, 5, 367.

[28] J. M. Seminario, Int. J. Quantum Chem. 1996, 60, 1271-1277.

[29] Y. Wang, D. J. Patel, Structure 1993, 1, 263-282.

[30] C. I. Bayly, P. Cieplak, W. Cornell, P. A. Kollman, J. Phys. Chem. 1993, 97, 10269-10280.

[31] C. I. Bayly, P. Cieplak, W. Cornell, P. A. Kollman, J. Phys. Chem. 1993, 97, 10269-10280.

[32] F. Wang, J.-P. Becker, P. C. \& F.-Y. Dupradeau, 2013.

[33] E. Vanquelef, S. Simon, G. Marquant, E. Garcia, G. Klimerak, J. C. Delepine, P. Cieplak, F.-Y. Dupradeau, Nucleic Acids Res. 2011, 39, W511-7.

[34] F.-Y. Dupradeau, A. Pigache, T. Zaffran, C. Savineau, R. Lelong, N. Grivel, D. Lelong, W. Rosanski, P. Cieplak, Phys. Chem. Chem. Phys. 2010, 12, 7821-39. 